UNIVERSIDADE DE SÃO PAULO

FACULDADE DE FILOSOFIA, CIÊNCIAS E LETRAS DE RIBEIRÃO PRETO DEPARTAMENTO DE COMPUTAÇÃO E MATEMÁTICA

EMERSON YOSHIAKI OKANO

Análise e caracterização de textos intencionalmente enganosos escritos em português usando métodos de processamento de textos 



\title{
Análise e caracterização de textos intencionalmente enganosos escritos em português usando métodos de processamento de textos
}

\author{
Versão Corrigida
}

Dissertação apresentada à Faculdade de Filosofia, Ciências e Letras de Ribeirão Preto (FFCLRP) da Universidade de São Paulo (USP), como parte das exigências para a obtenção do título de Mestre em Ciências.

Área de Concentração: Computação Aplicada.

Orientador: Prof. Dr. Evandro Eduardo Seron Ruiz

Ribeirão Preto-SP 



\section{Emerson Yoshiaki Okano}

Análise e caracterização de textos intencionalmente enganosos escritos em português usando métodos de processamento de textos. Ribeirão Preto-SP, 2020.

109p. : il.; $30 \mathrm{~cm}$.

Dissertação apresentada à Faculdade de Filosofia, Ciências e Letras de Ribeirão Preto da USP, como parte das exigências para a obtenção do título de Mestre em Ciências, Área: Computação Aplicada.

Orientador: Prof. Dr. Evandro Eduardo Seron Ruiz

1. Fake news. 2. Fake reviews. 3. textos enganosos. 

Emerson Yoshiaki Okano

Análise e caracterização de textos intencionalmente enganosos escritos em português usando métodos de processamento de textos

Modelo canônico de trabalho monográfico acadêmico em conformidade com as normas ABNT.

\begin{tabular}{c}
\hline $\begin{array}{c}\text { Orientador: } \\
\text { Orientador }\end{array}$ \\
\hline Professor \\
Convidado 1 \\
\\
\hline Professor \\
Convidado 2 \\
Ribeirão Preto-SP \\
2020
\end{tabular}



Este projeto de pesquisa é dedicado ao meu avô Yoshio Okano (in memoriam), que sempre esteve ao meu lado. 



\section{Agradecimentos}

Agradeço inicialmente a minha família, pelo amor, incentivo oferecido e apoio incondicional.

Ao Prof. Dr. Evandro Eduardo Seron Ruiz, meu orientador e amigo, pela oportunidade, suporte, correções e incentivos.

Ao meu amigo Sergio Naruhiko Sakurai que me apresentou a cidade de Ribeirão Preto e me ajudou muito durante este período.

A meus companheiros de laboratório, pelo bom convívio, amizade e boas discussões no decorrer desta etapa.

A todos meus amigos, que na minha despedida de Lins me deram um "oiwai", um gesto que nunca irei esquecer.

A todos os funcionários da USP que proporcionaram um ambiente criativo e amigável.

Ao convênio entre a Fundação de Amparo à Pesquisa do Estado de São Paulo (FAPESP) e Coordenação de Aperfeiçoamento de Pessoal de Nível Superior (CAPES), processo número 2018/03129-8 pelo apoio financeiro.

A todos que direta ou indiretamente fizeram parte da minha formação, o meu muito obrigado. 

"You can't connect the dots looking forward; you can only connect them looking backwards."

(Steve Jobs) 



\section{Resumo}

Atualmente a web é um ambiente em que pessoas postam e buscam informações sobre os mais diversos tópicos. No entanto, nem sempre uma informação encontrada na web é verdadeira já que alguns usuários maliciosos usam a web para disseminar informações falsas com o intuito de manipular ou enganar pessoas. Uma das maneiras de detectar estas informações falsas é através de processamento de textos. Atualmente há trabalhos direcionados à língua inglesa para identificação de textos enganosos, mas poucos trabalhos são voltados para a língua portuguesa. Neste trabalho, inicialmente foi criado um corpus paralelo de reviews de livros enganosos e foi feito um trabalho inicial de classificação automático do mesmo. Foi feito um estudo utilizando as research questions propostas por Hauch et al. para fazer uma análise psicolinguística do corpus de notícias falsas Fake.Br com o intuito de verificar as características mais relevantes para a classificação de fake news. Ainda utilizando o corpus Fake.Br treinamos algoritmos de aprendizagem de máquina supervisionados para fazer a classificação automática de fake news e utilizamos também um algoritmo de deep learning chamado Hierarchical attention network para verificar sua performance na detecção de fake news.

Palavras-chave: Fake news; Fake reviews; textos enganosos. 



\section{Abstract}

The web is an environment where people post and search any type of information on the most diverse topics. However, the information found on the web is not always truthful. There are malicious users who post deceptive information intending to manipulate or deceive people. One of the ways to detect false information is using text processing. Nowadays there are studies directed to the English language to identify deceptive texts, but there are few related works concerning the Portuguese language. In this work, initially, we created a parallel corpus of deceptive book reviews and used some machine learning algorithms to classify deceptive and truthful reviews. A study was made using the research questions proposed by Hauch et al. to do a psycholinguistic analysis of the fake news corpus Fake.Br to verify the most relevant features for fake news classification. Still using the Fake.Br corpus we trained supervised machine learning algorithms to automatically classify fake news and we also use a deep learning algorithm called Hierarchical attention network to verify its performance in fake news detection.

Keywords: Fake news; Fake reviews; deceptive texts. 



\section{Lista de figuras}

Figura 1 - Ilustração da divisão de textos enganosos. . . . . . . . . . . . . . . 26

Figura 2 - Ilustração das etapas de processamento deste projeto. . . . . . . . . . . 40

Figura 3 - Boxplot dos resultados obtidos variando o char n-gram. . . . . . . . . 53

Figura 4 - Boxplot dos resultados obtidos variando o char n-gram removendo stopwords. . . . . . . . . . . . . . . . . 5 54

Figura 5 - Boxplot dos resultados obtidos variando o char n-gram aplicando Stemming. . . . . . . . . . . . . . . . . . . 54

Figura 6 - Boxplot dos resultados obtidos variando o char n-gram removendo stopwords e aplicando stemming. . . . . . . . . . . . . 55

Figura 7 - Boxplot dos resultados obtidos variando o valor do parametro no below. 55

Figura 8 - Boxplot dos resultados obtidos variando o valor do parametro not above. 56

Figura 9 - Hierarchical Attention Network fonte: (YANG et al., 2016) . . . . . . . 66 



\section{Lista de tabelas}

Tabela 1 - Exemplo do resultado da aplicação do stemming e da lematização em palavras em Inglês. . . . . . . . . . . . . . . . . . . . . . 41

Tabela 2 - Matriz de confusão. . . . . . . . . . . . . . . . . 46

Tabela 3 - Resultados obtidos utilizando SVM e Random Forest sobre o corpus de reviews enganosos. . . . . . . . . . . . . . . . . . . . . . . 49

Tabela 4 - Resultados obtidos utilizando char 3-gram em textos truncados. . . . . 56

Tabela 5 - Rules of Thumb . . . . . . . . . . . . . . . 59

Tabela 6 - Atributos psicolinguísticos extraídos das notícias completas. . . . . . . 63

Tabela 7 - Atributos psicolinguísticos pertencentes a categoria Miscellaneous. . . . 64

Tabela 8 - Resultados obtidos utilizando atributos psicolinguísticos em textos truncados. . . . . . . . . . . . . . . . . . . . 6 64

Tabela 9 - Resultados obtidos utilizando HAN em textos completos. . . . . . . . 70

Tabela 10 - Resultados obtidos utilizando HAN em textos truncados. . . . . . . . 70 



\section{Sumário}

Introdução . . . . . . . . . . . . . . . . 25

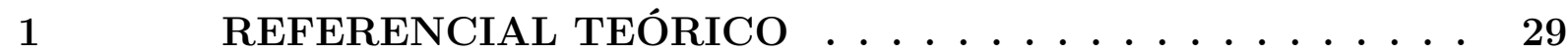

1.1 Sobre textos intencionalmente enganosos . . . . . . . . . 29

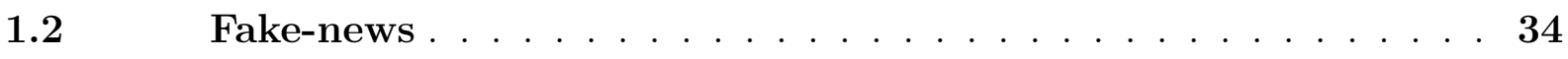

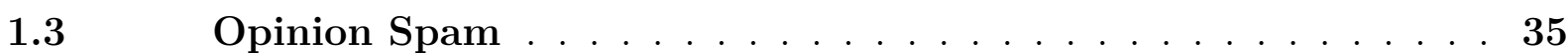

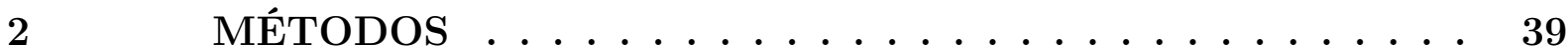

$2.1 \quad$ Modelo de detecção de textos enganosos . . . . . . . . . . 39

2.2 Pré-processamento . . . . . . . . . . . . . . . 40

$2.3 \quad$ Extração de características . . . . . . . . . . . . . 41

$2.4 \quad$ Classificadores . . . . . . . . . . . . . . . . . 43

3 CRIAÇÃO E CLASSIFICAÇÃO DE UM CORPUS SOBRE REVIEWS ENGANOSOS DE LIVROS . . . . . . . 47

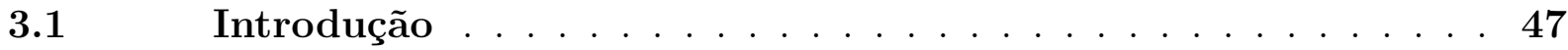

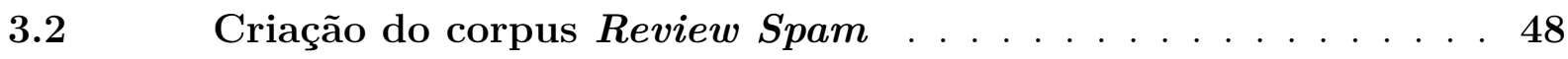

3.3 Resultados e discussão . . . . . . . . . . . . . . . . . . . . . 49

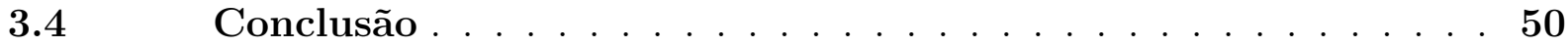

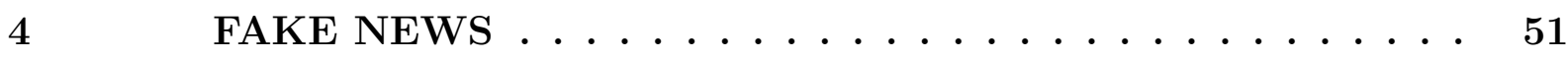

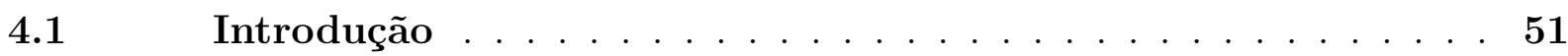

$4.2 \quad$ Fake.Br Dataset . . . . . . . . . . . . . . . . . 52

$4.3 \quad$ Análise do Fake.Br utilizando char n-gram . . . . . . . . 52

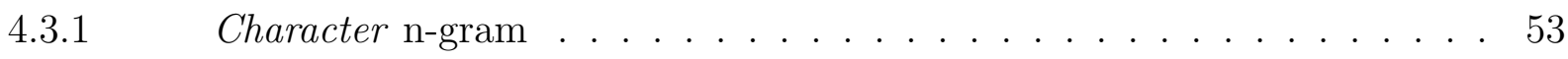

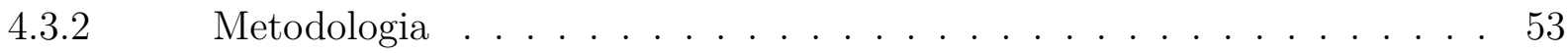

$4.3 .3 \quad$ Resultados . . . . . . . . . . . . . . . . . 55

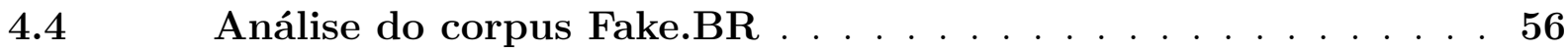

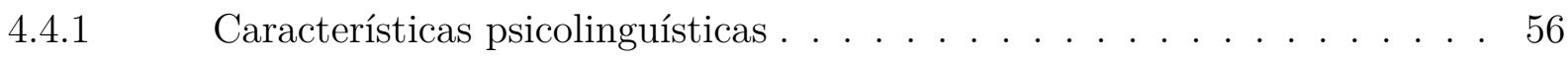

$4.4 .2 \quad$ Hedges effect size . . . . . . . . . . . . . . . . . . 59

$4.4 .3 \quad$ Resultados . . . . . . . . . . . . . . . . . . . 59

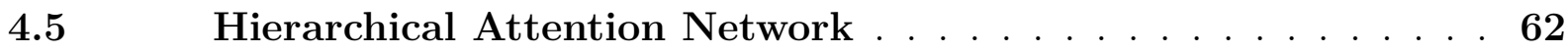

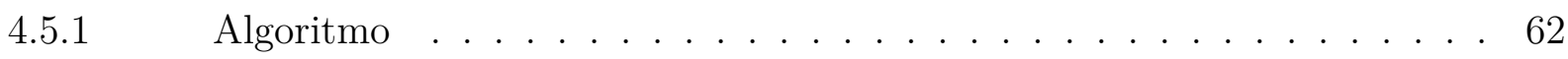

4.5.2 Configuração do modelo . . . . . . . . . . . . . . . . . 68

$4.5 .3 \quad$ Resultados . . . . . . . . . . . . . . . . . . . . 69

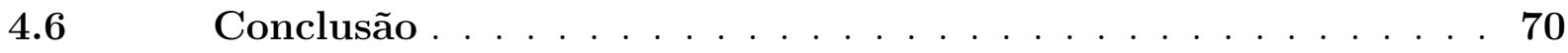




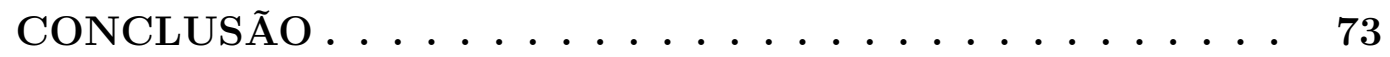

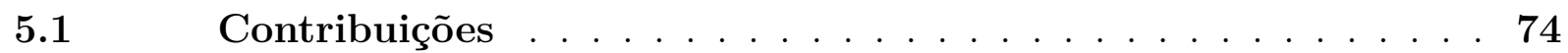

5.2 Trabalhos futuros . . . . . . . . . . . . . 74

Referências .................. 75

$\begin{array}{ll}\text { ANEXOS } & 79\end{array}$

ANEXO A - FINDING DECEPTIVE BOOK REVIEWS IN BRAZILIAN PORTUGUESE ............ 81

ANEXO B - USING LINGUISTIC CUES TO DETECT FAKE NEWS ON THE BRAZILIAN PORTUGUESE PARALLEL CORPUS FAKE.BR . . . . . . . . 89

ANEXO C - FAKE NEWS DETECTION ON FAKE.BR USING HIERARCHICAL ATTENTION NETWORKS . . . 99 


\section{Introdução}

\section{Contextualização}

O termo Inglês "deception", segundo o dicionário Michaelis, tem como tradução para o Português os termos fraude, logro, engano, ilusão. O mesmo termo segundo Zhou e Zhang (2008) é uma informação transmitida intencionalmente para criar uma falsa impressão ou conclusão. Em Português os termos "fraude" e "engano", e seus sinônimos, são usados como referentes a "deception" e "fake".

Atos de engano, deception, também têm sido usados em vários contextos ao longo da história humana (como na Segunda Guerra Mundial e na Guerra de Tróia) como recurso para aumentar a eficiência dos ataques, ou seja, fomentando o "logro". Hoje as mídias sociais fornecem novos ambientes e tecnologias para enganadores em potencial. Há muitos exemplos de pessoas sendo enganadas através das mídias sociais, algumas sofrendo consequências devastadoras em suas vidas sociais (TSIKERDEKIS; ZEADALLY, 2014).

Atualmente um grande número de pessoas desfruta de várias atividades on-line, tais como, como comprar e vender itens, disseminar ideias e buscar informações em geral. Tais informações buscadas ou postadas nem sempre são confiáveis já que algumas pessoas usam a web para disseminar informações falsas com o intuito de manipular ou enganar outros (HERNÁNDEZ-CASTAÑEDA et al., 2017). O site World Economic Forum ${ }^{1}$ classificou, em 2012, a disseminação de informações como um grande risco para a economia, mostrando a importância desta linha de pesquisa para a economia mundial.

Neste trabalho estudamos dois tipos de textos que fazem parte do mundo de textos enganosos, que são divididos de acordo com a Figura 1: a) Fake News: considerados aqui como notícias falsas (RUBIN et al., 2015), e; b) Opinion Spam: que são opiniões falsas sobre algum produto (OTT et al., 2011), também chamadas de fake reviews.

Recentemente muitos veículos de comunicação nacionais e internacionais anunciaram esforços para diminuir os efeitos das notícias falsas ou enganosas, ou mesmo revelar a verdade escondida nas fake news criando as páginas web conhecidas como páginas de fact

1 <https://www.weforum.org/agenda/2017/02/how-can-we-defeat-fake-news-automate-the-right-to-reply/ 


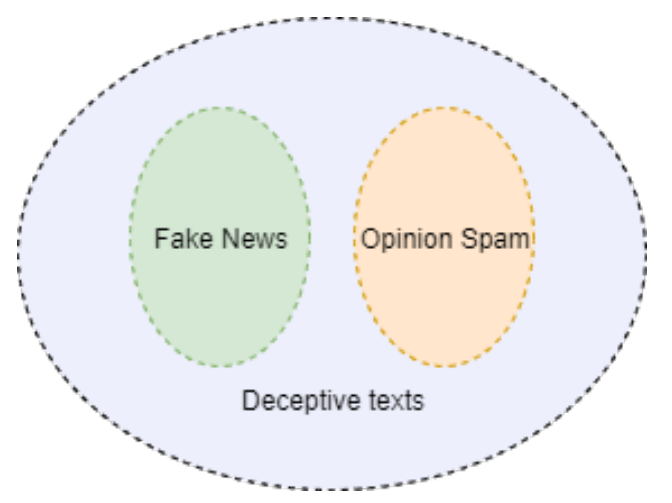

Figura 1 - Ilustração da divisão de textos enganosos.

checking. Como exemplos podemos citar o blog da revista semanal Veja, "Me engana que eu posto" ${ }^{2}$. Outro exemplo vem de um grupo de 37 veículos internacionais de mídia, capitaneados pela imprensa francesa, que se uniram para combater estas notícias danosas. Eles também anunciaram uma ferramenta para a identificação e combate as notícias falsas na web3 ${ }^{3}$ Seguindo esta mesma linha, várias empresas como Google, Facebook, Bing e Twitter juntaram suas forças para criar o The Trust Project ${ }^{4}$ que consiste em um consórcio internacional de organizações de notícias que estão colaborando com o objetivo de criar padrões de jornalismo com o objetivo de criar uma imprensa mais confiável.

Uma mensagem enganosa pode ser transmitida através de dois meios, assim chamados: a) Face-to-Face (F2F), que consiste em uma comunicação em que o autor e o receptor se relacionam diretamente um com o outro, e; b) Computer-Mediated Communication (CMC), que é uma comunicação mediada por computador em que o autor e o receptor se relacionam através de um computador. Dependendo do ambiente, a mensagem enganosa pode conter pistas diferentes que evidenciam seu caráter falso ou verdadeiro. A disponibilidade destas pistas num ambiente CMC é menor em relação a F2F devido ao fato da comunicação CMC ser baseada basicamente em mensagens de texto. Apesar das limitações, várias pistas ainda podem ser observadas em um ambiente CMC, como afirmam Ho et al. (2016).

Os primeiros trabalhos na detecção de deceptions vem das áreas da Psicologia e Psiquiatria (DePaulo et al., 2003) e eram focadas principalmente no ambiente F2F. Com a evolução da web e a grande busca por informações on-line observou-se um grande aumento da utilização do ambiente CMC, tais como, como chats online, mídias sociais, blogs de notícias, o que aumentou a necessidade de se desenvolver maneiras de validar informações nestes ambientes (HO et al., 2016).

\footnotetext{
$2<$ http://veja.abril.com.br/me-engana-que-eu-posto $>$

$3<$ http://internacional.estadao.com.br/noticias/geral,franca-cria-grupo-contra-fake-news, $70001683496>$

$4<$ https://thetrustproject.org/>
} 


\section{Motivação e Objetivos}

Atualmente há vários projetos com a finalidade de detectar textos enganosos utilizando métodos de processamento de textos (HAUCH et al., 2015). No entanto, a literatura carece de modelos para encontrar textos enganosos em Português, quase certo por ser uma área de pesquisa em Processamento de Língua Natural (PLN) ainda incipiente, como revelam os poucos artigos encontrados na revisão bibliográfica.

Tendo em vista a preocupação da sociedade acadêmica e econômica em relação à detecção de textos intencionalmente enganosos, escritos em Português, observou-se a necessidade de criar um modelo para a detecção deste tipo de texto utilizando processamento de textos abordando tanto características linguísticas quanto psicolinguísticas.

\section{Objetivo geral}

O objetivo geral deste trabalho é extrair e analisar características textuais em documentos intencionalmente enganosos, escritos em Português, com a finalidade de classificar estes textos como textos verdadeiros ou enganosos utilizando métodos de processamento de textos.

\section{Objetivos específicos}

- Coletar textos intencionalmente enganosos, escritos em Português, para criação de um corpus anotado;

- Pesquisar características e atributos linguísticos utilizados em trabalhos semelhantes para caracterização de textos intencionalmente enganosos;

- Pesquisar ferramentas e modelos já existentes para extração de características e atributos linguísticos de textos;

- Extrair características dos textos enganosos deste corpus criado;

- Utilizar um classificador para distinção entre textos enganosos e verdadeiros. Fazer uma análise detalhada dos resultados obtidos;

- Classificar os textos e comparar resultados com outros trabalhos e estudos semelhantes. 


\section{Método de pesquisa}

Neste projeto faremos uma pesquisa aplicada, que inicialmente será feito uma revisão bibliográfica sobre artigos que utilizam técnicas de processamento de linguagem natural para distinção de textos intencionalmente enganosos, analisando principalmente as técnicas utilizadas para a extração de características textuais e tentaremos criar um modelo utilizando estas característica para a detecção destes textos utilizando algoritmos de aprendizagem de máquina.

\section{Organização do trabalho}

Este documento está estruturado da seguinte forma: no Capítulo 1 apresentamos o referencial teórico, onde fazemos uma apresentação de vários artigos relacionados a textos intencionalmente enganosos, dividido em três categorias: 1. Textos intencionalmente enganosos em geral; 2. Fake news, e; 3. Opinion spam ou fake review. No Capítulo 2, apresentamos os métodos fundamentais utilizados em nosso trabalhos dos capítulos subsequentes, tais como métodos de pré-processamento do texto, extração de características e classificadores. A partir do Capítulo 3 nosso trabalho foi divido em duas partes que foram frutos de um desenvolvimento incremental que originaram três artigos. No Capítulo 3, apresentamos a criação de um corpus de reviews enganosos de livros e a classificação automática do mesmo utilizando algoritmos de aprendizagem de máquina também apresentados também no artigo: "Finding deceptive book reviews in Brazilian portuguese". No Capítulo 4 apresentamos três metodologias diferentes utilizadas para fazer a classificação entre fake news e notícias verdadeiras onde duas delas geraram artigos: "Using linguistic cues to detect fake news on the Brazilian Portuguese parallel corpus Fake.BR" e "Fake news detection on Fake.Br using hierarchical attention networks". No Capítulo 5 apresentamos as conclusões gerais do trabalho, contribuições e também eventuais trabalhos futuros. 


\section{Referencial Teórico}

Podemos dividir os trabalhos acadêmicos sobre textos intencionalmente enganosos em 3 grupos, que são:

1. Os trabalhos que abordam os textos intencionalmente enganosos em geral;

2. Os trabalhos direcionados especificamente para fake news; e

3. Os textos voltados para opinion spam ou fake reviews.

\subsection{Sobre textos intencionalmente engano- SOS}

O artigo de (HANCOCK et al., 2007) é seminal sobre este campo de estudos pois mostra como os autores fizeram um estudo investigativo sobre as mudanças no estilo linguístico em conversas baseadas em textos síncronos. Neste trabalho, os autores apresentam 7 hipóteses que distinguem textos enganosos de textos verdadeiros, que são: H1) Sujeitos mentirosos produzirão mais palavras durante conversas enganosas do que durante conversas verdadeiras; H2) Mentirosos farão mais perguntas durantes conversas enganosas em comparação a conversas verdadeiras; H3) Mentirosos utilizarão menos pronomes em primeira pessoa do singular em conversas enganosas; H4) Mentirosos utilizam mais sentimentos negativos em conversas enganosas; H5) Mentirosos utilizarão menos palavras exclusivas e termos de negação durante conversas enganosas em comparação com conversas verdadeiras; H6) Mentirosos evitarão frases de causalidades durante conversas enganosas; H7) Mentirosos utilizaram mais termos sensitivos durante conversas enganosas comparado a conversas verdadeiras. Os autores também verificaram duas questões de pesquisa:

RQ1 Os parceiros mudarão seus estilos durante conversas enganosas? Em caso afirmativo, como essas mudanças se relacionam no estilo linguístico dos mentirosos?; e 
RQ2 Como a motivação para enganar afeta o estilo linguístico de mentirosos e parceiros através de uma comunicação baseada em textos?

Para verificar estas hipóteses e questões de pesquisas, os autores reuniram 70 estudantes que foram divididos em pares do mesmo sexo para conversarem. Um dos estudantes era aleatoriamente atribuído com o papel de mentiroso o qual tinha como objetivo enganar seu parceiro. Neste artigo os autores verificam estas hipóteses e questões de pesquisas analisando as características psicolinguísticas obtidas utilizando a ferramenta LIWC $^{1}$ mostrando que utilizando esta ferramenta e algum classificador automático é possível diferenciar conversas enganosas de verdadeiras.

No artigo recente de Hauch et al. (2015) relata uma meta análise sobre as pistas linguísticas que podem auxiliar na detecção de textos enganosos. Neste estudo eles incluíram definições operacionais para 79 pistas descritas em 44 trabalhos acadêmicos as quais foram alocadas para seis perguntas de pesquisa, aqui formuladas em versão livre da língua inglesa para o português:

1. "Mentirosos sofrem maior carga cognitiva?"

2. "Mentirosos expressam menos certeza?"

3. a) "Mentirosos utilizam mais negação e mais palavras com carga emocional negativa?"

b) "Mentirosos utilizam menos palavras com carga emocional positiva?"

c) "Mentirosos utilizam mais ou menos palavras de carga emocional não especificada?"

4. "Mentirosos se distanciam dos eventos?"

5. "Mentirosos usam menos detalhes (sensoriais e contextuais)?"

6. "Os mentirosos referem-se menos aos processos cognitivos?"

Neste trabalho, Hauch e seus colaboradores mostraram aspectos linguísticos operacionais claros que levam a processos computacionais capazes de lidarem com estas perguntas. Mostraram também como estes processos podem ser integrados, ou seja, envolvendo as áreas de Psicologia, Cognição, Linguística e Computação.

O artigo de Appling, Briscoe e Hutto (2015) classifica textos enganosos em 4 categorias, de acordo com sua estratégia de classificação: 1) A falsificação, que consiste em mentiras, contradições ou distorções da verdade; 2) O exagero, que consiste em modificar a verdade utilizando superlativos; 3) A omissão, que é omitir parte da verdade, ou ainda, contar parte da verdade; e, finalmente, 4) O logro que é a troca de tópicos, informações 
irrelevantes ou equivocadas. Neste artigo, o autor classifica, por meio das características textuais, a estratégia utilizada pelo enganador e verifica que algumas estratégias são mais difíceis de identificar que outras alcançando $71 \%$ de precisão distinguindo exagero e falsificações utilizando características psicolinguísticas (utilizando o LIWC) e algumas características estruturais do texto (como número de palavras, tamanho médio das palavras, etc.) aplicadas a uma Random Forest para a classificação.

No artigo de (HANCOCK et al., 2004) os autores verificam como o modelo dos meios de comunicação afetam o ato de mentir. Neste artigo os autores verificaram 3 características do meio de comunicação: a) Sincronicidade da interação; b) A capacidade de gravação do meio, e; c) Se o emissor e o receptor da mentira estão distribuídos geograficamente, ou seja, não compartilham o mesmo espaço. Neste estudo foi pedido para as pessoas preencherem um formulário chamado Social Interaction and Deception (SID), que é formulado para documentar e categorizar interações e mentiras. Observando os resultados, os autores verificaram que apesar do número de mentiras ter sido maior através de F2F, Face-toFace, como meio de comunicação a maior proporção ocorreu utilizando telefone. Essa observação é consistente com a Social Distance Hypothesis (hipótese da distância social), que argumenta que pessoas escolhem um meio com menos recursos para mentir, a fim de evitar um desconforto associado a mentira.

No trabalho de Ho et al. (2016), é definida de maneira clara a diferença entre os ambientes de comunicação Face-to-Face (F2F) e Computer-Mediated Communication (CMC), como também a diferença entre mentiras espontâneas e as previamente planejadas. Tendo isto em vista, o autor propôs um modelo para a detecção de textos enganosos no ambiente CMC utilizando a ferramenta Linguistic Inquiry and Word Count (LIWC) para a extração de características textuais e uma Decision Tree como classificador. Neste trabalho, para obtenção de dados foi utilizado um jogo criado pelos autores chamado Real or Spiel onde dois jogadores entram em um chat onde é designado o papel de Speaker ou Detector. O Speaker pode assumir aleatoriamente dois papeis: Saint (conta verdades) ou Sinner (conta mentiras). O Detector no final de cada rodada deve determinar se o Speaker estava dizendo a verdade ou não. Este experimento foi feito com 20 pessoas em 10 rodadas do jogo. Analisando os dados obtidos, o autor observou que frases descritivas foram mais utilizadas por mentirosos e de maneira contrária a outros estudos, mostrou que autores de mentiras mostraram se envolver mais com a história. Segundo o autor, uma notável limitação de seu trabalho foi o pequeno número de amostras obtidas e este resultado poderia ser diferente caso a amostra de dados fosse maior.

Seguindo uma linha de pesquisa mais aplicada da Computação, envolvendo o conhecimento de redes sociais, Tsikerdekis e Zeadally (2014) relatam seus experimentos na classificação de comentários enganosos nestas novas mídias. Neste trabalho, os autores sugerem o que eles chamam de 'deception model'. Resumidamente, o modelo ('deception 
model') é mapeado em quatro componentes: a) um emissor (Sender) que é o individuo que emite a mensagem ou conteúdo enganoso; b) um canal de comunicação ("Communicationchannel") que é por onde o emissor envia a mensagem; c) Conteúdo da mensagem (Content); e d) um receptor, "receiver". Neste trabalho, o autor divide as deception de acordo com o componente utilizado para enganar o receptor. O Sender deception acontece quando se manipula a identidade do emissor (sender). O communication-channel deception acontece quando se manipula o canal de comunicação. O content deception é quando se manipula o conteúdo, falsificando informações, e é maneira mais utilizada. O autor cita também técnicas híbridas de deception formadas pela combinação de duas ou mais técnicas citadas anteriormente. Neste artigo os autores citam a importância da área de processamento de linguagem natural para detecção de textos enganosos citando alguns trabalhos que alcançaram uma acurácia na faixa de 80.4\%-98.6\%.

Ainda sobre textos enganosos, Mihalcea e Strapparava (2009), apresentaram três datasets anotados contendo textos verdadeiros e enganosos sobre assuntos polêmicos: aborto, pena de morte e melhor amigo. Esses datasets foram obtido utilizando o serviço Mechanical Turk ${ }^{2}$, que é um serviço de crowdsourcing da Amazon. Para os dois primeiros tópicos, as instruções foram para que os Turkers imaginassem que estivessem participando de um debate de 10 a 15 minutos para expressar sua opinião sobre o assunto, logo depois era pedido para os mesmos escreverem um texto expressando a opinião contrária a deles. No terceiro tópico (Melhores amigos) foi pedido para que os Turkers escrevessem a verdade sobre o que sentiam sobre seu melhor amigo, e depois foi pedido para pensarem em sobre uma pessoa que não era conhecida e descreve-la como se fosse seu melhor amigo. Foram coletados 100 textos verdadeiros e 100 textos falsos sobre cada tópico, cada texto possuindo em média 85 palavras. Neste artigo foram utilizadas as classes de palavras presentes no LIWC (TAUSCZIK; PENNEBAKER, 2010) como características. Para classificação foram utilizados os métodos SVM e Naïve Bayes. Inicialmente os autores utilizaram o 10-fold cross-validation em cada domínio obtendo uma média de $70 \%$ de acurácia. Os autores também testaram a acurácia dos classificadores utilizando o cross-topic classification, onde os textos de dois tópicos são utilizados para treinamento e os textos do terceiro tópico é utilizado como teste, onde foi obtido uma acurácia média de 59\%. Outra contribuição feita pelos autores foi o cálculo da dominância das classes das palavras provenientes do LIWC em cada domínio, enganoso e verdadeiro, obtendo resultados interessantes que mostraram que em ambos domínios três das classes dominantes eram relacionado a seres humanos, mas nos textos enganosos as classes de palavras relacionadas a humanos mostram distanciamento de si mesmo ao contrário das classes dominantes nos textos verdadeiros, no qual as classes das palavras são mais referentes a própria pessoa.

Em um artigo sobre detecção de mentiras no ambiente F2F, Abouelenien et al.

$2<$ https://www.mturk.com> 
(2017) utilizaram características linguísticas e características térmicas provenientes de uma câmera térmica. Neste experimento os participantes participaram de 3 cenários onde foram pedidos para fazerem depoimentos verdadeiros e falsos: A) Crime simulado: em que o sujeito é acusado de roubar um envelope contendo dinheiro; B) Aborto: quando foi pedido para os participantes fazerem um depoimento sobre o tema e; C) Melhor amigo: quando foi pedido para os participantes fazer uma descrição do melhor amigo. As características textuais utilizadas neste estudo foram os unigram codificados utilizando Term Frequency Inverse Document Frequency (tf-idf), características provenientes das classes do LIWC, os rótulos morfo-sintáticos (POS-tag), além das características térmicas coletadas foram: A) Segmentação e rastreamento de áreas de interesse; B) extração de características térmicas e C) Segmentos da face que indicam que a pessoa está mentindo. Os autores alcançaram resultados promissores utilizando as características linguísticas e térmicas simultaneamente e mostrou também que as características linguísticas, principalmente o unigram e o LIWC, desempenharam um papel muito importante na tarefa de classificação.

Utilizando uma abordagem mais estatística temos o artigo escrito por Zhou, Shi e Zhang (2008). Neste artigo os autores desenvolveram um Statistical Language Model (SLM) para detectar textos enganosos. Segundo os autores um SLM representa a distribuição de probabilidade sobre uma sequência de tokens $S$ que reflete o quão frequentemente $S$ ocorre. Neste artigo os autores desenvolveram dois SLM: a) um utilizando textos enganosos e; b) outro utilizando textos verdadeiros. O texto desconhecido é classificado verificando os dois modelos e escolhendo o modelo com o menor valor de perplexidade. Neste artigo foi utilizado um dataset contendo email e mensagens instantâneas verdadeiros e enganosos e para validar o resultado foi utilizado o 10-fold cross-validation obtendo uma acurácia média de $65.96 \%$.

Em um artigo voltado para técnicas de criação de um corpus voltado para textos enganosos, Gokhman et al. (2012) publicaram um survey apresentando tanto as técnicas mais tradicionais quanto as non-gold standart. Começando pelas técnicas mais tradicionais que são divididas em duas: a) Mentiras sancionadas: que são mentiras obtidas através de instruções explícitas para a pessoa mentir ou não mentir; b) Mentiras não sancionadas: que são mentiras coletadas sem uma instrução explícita ou permissão vinda do pesquisador. Uma alternativa as técnicas tradicionais temos as non-gold standart: a) Anotações manuais de mentiras: Diário de mentiras e métodos de self-report; b) Rotulados heuristicamente; c) Não rotulados. Neste artigo os autores apresentam também o crowdsourcing que consiste em uma colaboração coletiva onde o pesquisador define os parâmetros que ele deseja e as pessoas seguem esses parâmetros, este método geralmente e utilizado para gerar mentiras sancionadas. 


\section{$1.2 \quad$ Fake-news}

O artigo da Rubin et al. (2015) apresenta-se como uma artigo ontológico sobre o tema de fake news. Nele os autores apresentam alguns padrões a serem seguidos para elaborar um corpus de fake news:

1. Disponibilidade de instâncias verdadeiras e falsas: métodos preditivos são capazes de detectar padrões em instâncias verdadeiras e falsas. Achar a contraparte de notícias autênticas é o maior desafio:

2. Acessibilidade ao texto em forma digital;

3. Maneira de verificar a veracidade da notícia: É necessário uma maneira de verificar de forma confiável a veracidade das notícias;

4. Homogeneidade no tamanho das notícias;

5. Homogeneidade no tipo das notícias: O corpus deve ser separado por gênero da notícia (e.g., notícias de ultima hora, editoriais, op-eds ${ }^{3}$ ) e tópicos (economia, política, saúde) e serem escritos por tipos de autores semelhantes (e.g., jornalistas profissionais, humoristas, cidadãos comuns);

6. Janela de tempo predefinida:

7. O tipo de canal no qual a notícia foi transmitida (e.g., site de notícias sensacionalistas, jornal acreditado pelo público, site de notícias absurdas):

8. As preocupações pragmáticas incluem custos de direitos de cópia, disponibilidade pública, facilidade de obtenção, volume de dados global adequado, graus de divulgação e privacidade dos escritores.

9. A linguagem e cultura são fatores silenciosos que geralmente são esquecidos.

Este artigo também faz a separação das fake news em três tipos: a) Serious fabrication, que são fake news de fabricação séria, ou seja, reportagens fraudulentas com intenção de enganar os leitores com alguma finalidade; b) Large-scale hoax, que são boatos ou brincadeiras em largas escalas e, finalmente; c) Humorous fakes, que seriam notícias que os leitores estão cientes do exagero, como sátiras e exageros.

Em um survey voltado para formas de detecção de Fake News, Conroy et al. (2015) mostram o estado da arte nesta área. Neste survey o autor separa as técnicas utilizadas na área de verificação de notícias enganosas em duas vertentes: a) Aproximação

3 Página de um jornal onde são expressas opiniões de colunistas e comentaristas. 
linguística: que tem como objetivo, procurar por características chamadas neste survey de predictive deception cues no conteúdo da mensagem; b) Aproximação por redes: que vem para complementar a aproximação baseada em conteúdo utilizando propriedades e comportamento de redes. Neste survey os autores recomendam utilizar uma aproximação híbrida para melhor acurácia.

No artigo de Rubin et al. (2015), os autores fizeram um trabalho mais voltado para a classificação das notícias entre notícias verdadeiras e fake news. Neste artigos os autores usaram como research question a seguinte questão: "Como as relações retóricas entre as partes constituintes do discurso diferem entre verídica e enganosa?". Para esta tarefa os autores utilizaram uma ferramenta chamada Rhetorical Structure Theory (RST) utilizando o Vector Space Model (VSM) para a identificação das relações obtidas pelo RST e como classificador foi utilizado gCLUTO clustering package. Como dataset os autores utilizaram transcritos de um programa da rádio americana NPR, Natiaonal Public Radio, chamado "Buff the Listener" que contém em cada programa três notícias ligadas tematicamente sendo uma verdadeira e as restantes falsas. Neste artigo os autores não conseguiram um bom desempenho na tarefa de classificação, obtendo uma acurácia de $63 \%$.

Em um artigo recente, Monteiro et al. (2018) construíram o primeiro corpus paralelo sobre fake news em Português contendo 7.200 notícias, onde 3.600 são fake news e 3.600 são notícias verdadeiras sobre o mesmo tema das fake news, este corpus foi montado inicialmente coletando e checando apenas fake news que foram publicadas em um intervalo de dois anos (01/2016 até 01/2018). Após coletarem as fake news eles iniciaram a coleta semi-automática das notícias verdadeiras utilizando um web crawler extraindo notícias das principais agências de notícias do Brasil (G1, Folha de São Paulo e Estadão) e utilizaram a medida de similaridade léxica para selecionar as notícias mais similares as fake news coletadas e após essa coleta eles fizeram uma verificação manual para garantir que as notícias verdadeiras fosse do mesmo assunto das fake news coletadas. Além da coleta de dados, Monteiro et al. (2018) fizeram também uma análise bem detalhada sobre o corpus verificando suas principais características e também utilizaram aprendizagem de máquina para fazer a classificação automática de fake news obtendo uma acurácia de $89 \%$ utilizando bag of words e emotividade.

\subsection{Opinion Spam}

O survey escrito por Heydari et al. (2015) é leitura obrigatória para o tema Opinion Spam. Neste artigo são mostradas e definidas as três técnicas utilizadas para detecção de Opinion Spam, como propostas pelos autores. A saber: A) Encontrar cópias: consiste em encontrar reviews similares publicados pelo mesmo autor ou por diferentes pessoas. A similaridade entre os reviews pode ser analisada por duas perspectivas: 1) duplicação de texto, e; 2) 
similaridade conceitual entre os reviews; B) Métodos baseados em conteúdo: que pode ser dividido em três frentes: 1) Genre identification: Utiliza a distribuição dos POS-tags; 2) Características psicolinguísticas: Técnica que busca atribuir significados a palavras utilizadas no texto; 3) Categorização de textos: modelar o conteúdo do review utilizando n-gram; C) Outros métodos: em que os autores apresentam outros métodos utilizados para a detecção de opinion spam. Neste survey os autores apresentam também técnicas de detecção de Spammers utilizando teoria grafos.

No survey escrito por Crawford et al. (2015) os autores classificam Opinion Spam em três categorias: A) Untruthful opinions: que consiste nos reviews escritos com intenção de enganar o consumidor e dentre os três tipos é o que causa maior preocupação, pois prejudica o sistema de revisão online; B) Reviews on Brands: onde os reviews se focam na marca ou no vendedor do produto, mas falha ao fazer uma descrição do mesmo e C) Non-Reviews: Reviews que contém textos ou anúncios não relacionados. Além da categorização dos opinion spam os autores apresentaram também uma tabela comparando vários trabalhos contendo: nome dos autores, dataset utilizado, técnicas utilizadas para a extração de características, classificadores, métrica de avaliação do resultado, resultado e a complexidade do método utilizado por outros autores posteriormente para detecção de reviews falsos. Dentre as técnicas temos: bag-of-words, LIWC, frequência de parts of speech (POS), estilométricas e sintáticas, além de características vindas de metadados do review e não do texto.

No artigo de Ott et al. (2011) os autores criaram um corpus anotado sobre opiniões falsas sobre hotéis de Chicago, para formar este corpus foi utilizado opiniões consideradas verdadeiras do site TripAdvisor e para os textos enganosos foi utilizado o serviço de crowdsourcing chamado Mechanical Turk. Este corpus possui 1600 opiniões, sendo eles: 400 verdadeiras positivas, 400 verdadeiras negativas, 400 enganosas positivas e 400 enganosas negativas. Utilizando este corpus, os autores fizeram um experimento para verificar a humana na tarefa de detectar opiniões enganosas, em que o ser humano obteve uma acurácia média de $57 \%$. Os autores também verificaram métodos automáticos para detecção de opiniões enganosas utilizando três aproximações: a) Genre identification: que se trata de verificar a distribuição de frequência dos Part-Of-Speech; b) Características psicolinguísticas: características retiradas do textos utilizando a ferramenta Linguistic Inquiry and Word Count (LIWC) e c) Categorização de textos: características retiradas através de n-grams. Para a classificação foram utilizados os algoritmos Naïve Bayes e Support Vector Machine. Observando os resultados obtidos através da detecção automática de opiniões enganosas os autores observaram que o modelo treinado utilizando bigramas + LIWC como características e SVM para classificação obteve a melhor acurácia 89,8\% mostrando que a combinação das características baseadas em n-gram e baseadas em psicolinguística pode obter resultados ligeiramente melhores do que sozinhos. 
Em um trabalho mais voltado a Deceptive Opinion Spam, que consiste em reviews fictícios que são escritos deliberadamente para soarem como autênticos, escritos com o intuito de enganar os leitores, Ott, Cardie e Hancock (2013) classificam as opiniões em verdadeiras ou enganosas utilizando n-gram e SVM, Support Vector Machine. Neste trabalho foi utilizado uma base de dados chamada OpSpam (OTT et al., 2011) contendo 1600 reviews sobre 20 dos hotéis mais populares de Chicago. Dentre esses 1600 reviews, 400 são verdadeiros e positivos, 400 verdadeiros e negativos, 400 enganosos e positivos e, 400 enganosos e negativos. Os reviews enganosos foram obtidos utilizando Mechanical Turk. Neste trabalho, os autores demonstraram a relação entre a análise de sentimentos e deteç̧ão de textos enganosos. Os autores obtiveram precisão aproximada de $89 \%$ para a deteç̧ão de reviews enganosos.

Em um artigo mais recente, Hernández-Castañeda et al. (2017) avaliou o uso de Support Vector Networks (SVN) na detecção de deceptions. Neste trabalho os autores testaram além das ferramentas mais tradicionais já utilizadas como o LIWC, e Word-Space Model (WSM) eles utilizaram também outra abordagem utilizando topic modeling com a ferramenta Latent Dirichlet Allocation (LDA). Eles utilizaram três corpora diferentes para os quais os modelos foram treinados de três maneiras diferentes: a) one-domain setting, em que o treinamento e teste do modelo foram feito separadamente para cada corpus e foi obtido uma precisão média de $86 \%$, b) mixed-domain setting em que o corpus foi construído através de uma mistura de todos os três corpora e, para tanto, obtiveram uma precisão média de $75 \%$, e; c) cross-domain setting em que o modelo foi treinado sob uma mescla dos três corpora e foram executados testes separadamente em cada corpus. Os autores obtiveram uma precisão entre $52 \%$ e $64 \%$.

Vimos neste Capítulo alguns comentários sobre os principais artigos científicos pesquisados na grande área de notícias enganosas. A seguir, no Capítulo 2, veremos quais os principais métodos que são aplicados no tratamentos destes textos a fim de obter uma classificação dos mesmos. 



\section{Métodos}

Neste capítulo apresentaremos alguns métodos básicos utilizados tanto na parte de préprocessamento quanto na parte de validação de resultados. Aqui são apresentados alguns métodos comuns utilizados nos capítulos posteriores. Em geral os métodos automáticos de detecção de textos enganosos são divididos em três etapas: pré-processamento, extração de características e o classificador, que serão apresentados neste capítulo.

\subsection{Modelo de detecção de textos enganosos}

Nesta seção será apresentado um fluxograma do modelo de detecção de textos enganosos que consiste em três etapas: a) pré-processamento; b) extração de características, e; c) classificação, como pode ser observado na Figura 2, página 40.

O primeiro passo do nosso modelo é o pré-processamento dos dados, etapa na qual ocorrerá o tratamento e a preparação dos dados, tais como a remoção de caracteres indesejados, a remoção de stop words e a normalização do texto. Esta é uma etapa muito importante para o funcionamento correto das etapas posteriores.

O segundo passo é a extração de características textuais, quando ocorre o processamento do texto para a extração das características. Nesta etapa utilizaremos como base trabalhos de outros autores citados co capítulo anterior.

O último passo, mas não menos importante, é o classificador. Como classificador utilizaremos um algoritmo de aprendizagem de máquina supervisionada para a classificação dos textos. Nesta etapa pretendemos utilizar o 10-fold cross validation e o F1-score para avaliação dos resultados. 


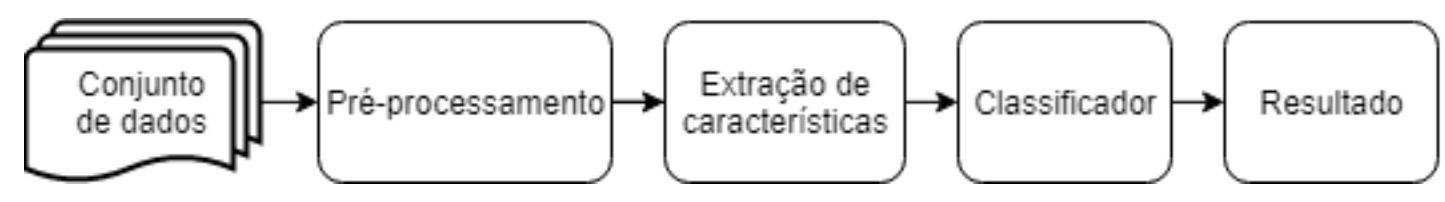

Figura 2 - Ilustração das etapas de processamento deste projeto.

\subsection{Pré-processamento}

Nesta etapa apresentaremos ferramentas que normalmente são utilizadas na fase de pré-processamento, que é a fase onde o texto é preparado para a fase de extração de características.

\section{Tokenização}

A tokenização, ou segmentação de palavras, é um dos primeiros passos feito na tarefa de pré-processamento de um texto e tem como finalidade dividir um documento textual em unidades (tokens) (JURAFSKY; MARTIN, 2008). Estas unidades nem sempre correspondem a apenas uma palavra do texto, as vezes um token pode ser uma palavra composta como 'guarda-roupas', ou uma data, como esta '01/01/2018'.

Um dos grandes desafios para a tokenização é a maneira de dividir os documentos em tokens, pois muitas vezes dividir os tokens utilizando os pontos, virgulas e espaços nem sempre é a maneira mais precisa, por exemplo, as vezes pode ocorrer de separar um número decimal em dois como 29.1.

Na biblioteca Natural Language Toolkit (BIRD; LOPER; KLEIN, 2009), também chamada de NLTK, já foram desenvolvidas várias funções para a tokenização que devem ser escolhidas de acordo com sua necessidade. Um exemplo muito interessante de tonekizador do NLTK é o TweetTokenizer, que é um tokenizador projetado para textos principalmente do Twitter, mas funciona muito bem para textos escritos em redes sociais em geral. A grande vantagem deste tokenizador é que ele reconhece sequências de símbolos que formam emoticons, como por exemplo, ':D', e também reduz o tamanho de algumas strings quando pessoas utilizam uma repetição de alguma letra para demonstrar exagero por exemplo quando uma pessoa escreve "Muuuuuuuuuuuito" ao transformar esta palavra em um token ela é reduzida para "Muuuito" o que reduz significativamente o número de tokens diferentes presentes em um corpus. 


\section{Stop-words}

Stop-words são palavras que podem ser consideradas irrelevantes para a tarefa que se deseja fazer, geralmente são palavras que não possuem valor semântico. Geralmente fazem parte das listas de stop-words palavras como conjunções, preposições, pronomes e artigos.

A remoção das stop-words de um textos, além de diminuir o número de tokens diferentes presentes o que diminui o tamanho do vetor de características, geralmente aumenta a precisão obtida pelo classificador.

\section{Stemming e Lematização}

Tanto o stemming quanto a lematização são algoritmos projetados para reduzir as palavras flexionadas e as derivações de cada palavra para uma base comum. Como por exemplo as palavras "computadores" e "computador" após a utilização do stemming se transformam na palavra "comput".

O algoritmo stemming funciona removendo sufixos ou flexões no final da palavra. O corte da palavras pode ser bem sucedido em algumas ocasiões, mas nem sempre. A Tabela 1 mostra algumas limitações. O algoritmo de lematização, por outro lado, considera a morfologia das palavras, ou seja, ele retorna a base ou a forma em que a palavra é encontrada no dicionário, conhecida como lema.

Na Tabela 1 podemos observar claramente a diferença entre o stemming e a lematização, assim como a limitação do stemming.

\begin{tabular}{lll}
\hline Palavra & Stemming & Lemmatization \\
\hline geese & gees & goose \\
presumably & presum & presumably \\
provision & provis & provision \\
\hline
\end{tabular}

Tabela 1 - Exemplo do resultado da aplicação do stemming e da lematização em palavras em Inglês.

\subsection{Extração de características}

Nesta seção apresentaremos alguns modelos de extração de características utilizados ao longo do projeto. Neste capítulo apresentaremos apenas os métodos mais utilizados para classificação, nos capítulos seguintes apresentaremos outros métodos utilizados nos artigos publicados durante o mestrado. 


\section{Bag of words (BOW)}

Um dos modelos mais simples e mais utilizado é o bag of words. Neste modelo um texto é representado como uma "sacola" de palavras, ou seja, guardamos apenas o número de ocorrência das palavras não levando em conta a ordem das palavras. Muitos autores (OTT et al., 2011; HERNÁNDEZ-CASTAÑEDA et al., 2017; MONTEIRO et al., 2018) utilizaram o bag of words e obtendo resultados significativos em seus experimentos apesar da simplicidade do modelo.

\section{Linguistic Inquiry and Word Count (LIWC)}

Como o visto na seção de fundamentação teórica Capítulo 1, vários trabalhos utilizaram características psicolinguísticas para a detecção de textos enganosos. Uma ferramenta muito utilizada neste âmbito é o Linguistic Inquiry and Word Count (LIWC) (TAUSCZIK; PENNEBAKER, 2010).

O LIWC é uma ferramenta baseada na contagem de palavras, em que cada palavra é separada em categorias que representam um significado psicológico. Esta ferramenta é composta por dois componentes centrais: a) o componente de processamento e; b) os dicionários. O componente de processamento é o software em si, que abre os arquivos e processa o texto palavra a palavra. Cada palavra processada é comparada com os dicionários. Já os dicionários são o coração do LIWC. Os dicionários se referem ao grupo de palavras que define uma categoria particular. Inicialmente o LIWC foi desenvolvido para calcular a porcentagem de palavras positivas e negativas em um texto. Após algumas semanas, o número de categorias foi expandido de 2 para 80 categorias, muitas dessas categorias são voltadas para área da Psicologia, o que é muito interessante para este nosso trabalho.

\section{Latent Dirichlet Allocation (LDA)}

Outro modelo recentemente utilizado para a distinção de textos foi o LDA (Latent Dirichlet allocation) (BLEI; NG; JORDAN, 2003). O LDA consiste de um modelo probabilístico generativo bastante funcional para coleções de dados discretos, como são as coleções de textos. Este modelo representa cada documento como uma coleção de diferentes tópicos. Os tópicos são estruturas com valor semântico e que, no contexto de mineração de texto, formam grupos de palavras que frequentemente ocorrem juntas (FALEIROS; LOPES, 2016). Assim, cada tópico é representado por conjunto de palavras que mantém uma ligação entre elas. Palavras, por sua vez, podem ser escolhidas baseadas em probabilidade. O modelo assume que cada documento é formado palavra a palavra ao selecionar aleatoriamente um 
tópico e uma palavra para esse tópico. Como resultado, cada documento pode combinar diferentes tópicos.

Assumindo este modelo generativo para uma coleção de documentos, o LDA analisa o conjunto de documentos para fazer a engenharia reversa deste processo e encontrar o conjunto mais provável de tópicos. Ao contrário do LIWC, o LDA gera os grupos de palavras (tópicos) automaticamente. Portanto o LDA é um método mais flexível quanto a linguagem se o relacionarmos com o LIWC que baseia-se num léxico criado apenas para a língua inglesa.

\section{Vector space model (VSM)}

No Vector Space Model documentos são representados como um vetor de características representando os termos (palavras) presentes no documento (JURAFSKY; MARTIN, 2008). O valor de cada característica é chamado de term weight e normalmente é a função de frequência do termo no documento.

No caso da utilização do VSM para a representação textos utilizando bag-ofwords podemos dizer que, assumindo que nosso dataset contenha $N$ documentos e $T$ tokens distintos podemos dizer que o documento $D_{i}$ pode ser representado por $D_{i}=$ $\left[t w_{i 0}, t w_{i 1}, t w_{i 2}, \cdots, t w_{i T},\right]$, onde $t w_{i t}$, no caso da utilização com bag-of-words utilizando a frequência das palavras, utiliza a frequência da palavra $t \in[1, T]$ no documento $i \in[1, N]$.

\subsection{Classificadores}

Nos trabalhos mais recentes na área de detecção de textos intencionalmente enganosos observou-se a necessidade de utilização de um algoritmo de aprendizagem de máquina para fazer a classificação dos textos (HERNÁNDEZ-CASTAÑEDA et al., 2017; ZHOU; ZHANG, 2008).

\section{Naïve Bayes}

O algoritmo Naïve Bayes (MURPHY, 2006) é um conjunto de algoritmos de aprendizagem de máquina supervisionado muito utilizado em classificação de textos. Este conjunto de algoritmos é baseado no teorema de Bayes com a ingênua "Naïve" suposição de independência entre as características. Resumindo o classificador Naïve Bayes assume que a presença de uma característica particular em uma classe não esta relacionada com a presença de qualquer outra característica. 
O algoritmo Naïve Bayes em sua forma mais simples segue a equação:

$$
P\left(y \mid x_{1}, \ldots, x_{n}\right)=\frac{P(y) \prod_{i=1}^{n} P\left(x_{i} \mid y\right)}{P\left(x_{1}, \ldots, x_{n}\right)}
$$

dado que $P\left(x_{1}, \ldots, x_{n}\right)$ é constante para todas as entradas podemos simplificar a equação para:

$$
\hat{y}=\arg \max _{y} P(y) \prod_{i=1}^{n} P\left(x_{i} \mid y\right)
$$

em que $x$ é o vetor de características e $y$ são as classes.

\section{Support Vector Machine (SVM)}

Um dos algoritmos mais utilizados para a classificação de textos (OTT et al., 2011; JINDAL; LIU, 2008; HERNÁNDEZ-CASTAÑEDA et al., 2017; FORNACIARI; POESIO, 2014) é o Support Vector Machine (SVM). Os SVMs são embasados na teoria do aprendizado estatístico, desenvolvido por Vapnik (VAPNIK, 1999). Essa teoria estabelece uma série de princípios que devem ser seguidos na obtenção de classificadores com boa generalização, definida como a sua capacidade de prever corretamente a classe de novos dados do mesmo domínio em que o aprendizado ocorreu (LORENA; CARVALHO, 2007).

De modo bem simplificado, podemos dizer que o SVM classifica os dados utilizando um hiperplano para separar os dois tipos de dados, buscando maximizar a distância entre os pontos mais próximos em relação a cada uma das classes. Um exemplo de SVM é o Linear SVM, que aprende um vetor de pesos $\vec{w}$ e um bias $b$, onde o item a ser classificado possui um vetor de características $\vec{x}$ e é classificado por:

$$
\hat{y}=\operatorname{sign}(\vec{w} \cdot \vec{x}+b)
$$

\section{Random Forest}

Random forest é um algoritmo de aprendizado de máquina supervisionado baseado no método de ensemble chamado bagging. Inicialmente são criadas amostras aleatórias do dataset de treinamento, em seguida são treinadas árvores de decisões para cada amostra do dataset criado e finalmente os resultados da classificação consiste na combinação destas árvores de decisões, utilizando um sistema de votação. Ou seja, mais especificamente a random forest é uma combinação de árvores de decisões, de modo que cada árvore depende de um vetor aleatório amostrado independentemente do dataset com a mesma distribuição para todas as árvores de decisão na floresta (BREIMAN, 2001). 


\section{Métodos de avaliação dos resultados}

Nesta seção apresentaremos métodos de avaliação dos resultados utilizados ao decorrer do trabalho.

\section{K-fold cross-validation}

O K-fold cross-validation é um método de avaliação do modelo de aprendizagem de máquina visando a capacidade de generalização do mesmo.

O método $K$-fold cross-validation consiste em dividir o dataset em $K$ folds do mesmo tamanho, para cada fold utilizado para validação utilizamos $K-1$ fold para treinamento. É importe mencionar que os dados do fold utilizado para a validação do resultado não são utilizados para o treinamento.

\section{Precisão, Revocação, Medida-F e Acurácia}

A Precisão, Revocação, Medida-F e Acurácia são medidas muito utilizados na literatura para avaliação dos resultados obtidos por algoritmos de aprendizagem de máquina.

Inicialmente apresentamos os termos verdadeiros positivos $(V P)$, verdadeiro negativo $(V N)$, falso positivo $(F P)$ e falso negativo $(F N)$, que são obtidos seguindo as relações mostradas na Tabela 2 .

A acurácia é a porcentagem de acertos obtido pelo classificador. É calculada pela Fórmula 2.1:

$$
\text { Acurácia }=\frac{(V P+V N)}{(V P+V N+F P+F N)}
$$

A precisão, ou especificidade, é a porcentagem de acertos obtidos em todos os documentos classificados como positivos, ou seja, o número de documentos classificados corretamente como positivo dividido pelo número total de instâncias previstas como positivas. Veja a Fórmula 2.2.

$$
\text { Precisão }=\frac{V P}{(V P+F P)}
$$

Revocação, ou sensibilidade, é a porcentagem de classificados positivos corretamente dentre todos os positivos reais, como o mostrado na Fórmula 2.3.

$$
\text { Revocação }=\frac{V P}{(V P+F N)}
$$


A medida-F é a média harmônica entre precisão e revocação,

$$
\text { Medida-F }=2 \times \frac{\text { Precisão } \times \text { Revocação }}{\text { Precisão }+ \text { Revocação }}
$$

Para a análise de resultados utilizaremos estas medidas e faremos uma comparação com outros trabalhos na língua inglesa para verificar a eficiência do método utilizado.

\begin{tabular}{|c|c|c|c|}
\hline & \multicolumn{2}{|c|}{ Previsto } \\
\hline & & Positivo & Negativo \\
\hline \multirow{2}{*}{ Real } & Positivo & Verdadeiro Positivo $(V P)$ & Falso Negativo $(F N)$ \\
\hline & Negativo & Falso Positivo $(F P)$ & Verdadeiro Negativo $(V N)$ \\
\hline
\end{tabular}

Tabela 2 - Matriz de confusão. 


\section{Criação e classificação de um corpus sobre reviews enganosos de livros}

O presente capítulo apresentará a criação e classificação automático de um corpus de reviews enganosos de livros. Neste capítulo será apresentado os métodos utilizados tanto para a criação quanto para a classificação do corpus Review spam. Os dados apresentados neste capítulo foram publicados em um student research workshop no International Conference on the Computational Processing of Portuguese PROPOR 2018.

\subsection{Introdução}

Atualmente um grande número de pessoas desfrutam de várias atividades on-line, tais como, como comprar e vender itens, disseminar ideias e buscar informações em geral. Tais informações buscadas ou postadas nem sempre são confiáveis já que algumas pessoas usam a web para disseminar informações falsas com o intuito de manipular ou enganar outros (HERNÁNDEZ-CASTAÑEDA et al., 2017). O site World Economic Forum ${ }^{1}$ classificou, em 2012, a disseminação de informações como um grande risco para a economia, mostrando a importância desta linha de pesquisa para a economia mundial.

Como mostrado anteriormente um dos tipos de deceptions são os deceptive reviews que Dixit e Agrawal (2013) chamam também de opnion spam, que são divididos em três grupos: (1) Untruthful reviews: Que são reviews falsos que tem a clara intenção de enganar o público; (2) Reviews on brands que são reviews que contém comentários que são pertinente apenas a marca do produto, a loja ou ao vendedor, mas não contem nada útil sobre o produto; (3) Non reviews: São textos que não apresentam nada relacionado ao produto. O segundo (2) e terceiro (3) tipo são chamados de disruptive opinion spam, esses dois tipos de opinion spam representam pouca ameaça para os humanos pois eles

$1<$ https://www.weforum.org/agenda/2017/02/how-can-we-defeat-fake-news-automate- $\%$ the-right-to-reply/> 
conseguem facilmente identificar esses falsos reviews. O primeiro (1) tipo é o mais confuso e que representa maior perigo para a humanidade, pois são muito difíceis para os humanos identificarem como o mostrado por Ott et al. (2011) onde os seres humanos conseguiram uma acurácia de aproximadamente $58.1 \%$.

Neste capítulo apresentaremos a criação de um corpus sobre opinion spam, mais especificamente sobre untruthful reviews, sobre sobre livros. Apresentaremos também a utilização de algoritmos de aprendizagem de máquina supervisionados para a classificação automática dos reviews.

\subsection{Criação do corpus Review Spam}

Para a criação do corpus de reviews enganosos sobre livros utilizamos o método goldstandard proposto por Gokhman et al. (2012). Em seu artigo Gokhman et al. apresentou métodos para a criação de corpus de textos intencionalmente enganosos sendo os dois principais o sancionado ou não-sancionado, onde o sancionando os participantes do experimento para a criação do corpus são instruídos explicitamente para mentir ou não, e o não-sancionado os participantes mentem de acordo com sua vontade.

Considerando os métodos apresentados por Gokhman et al. (2012) dividimos a coleta de dados em duas partes:

- Reviews enganosos: Coletamos reviews engansoso de livros utilizando o método sancionado proposto por Gokhman (GOKHMAN et al., 2012), no qual é explicitamente pedido aos participantes para escreverem um review enganoso. Os reviews enganosos foram coletados utilizando o Google Forms ${ }^{2}$. Este serviço foi utilizado para a coleta de reviews enganosos sem custo algum. Neste ambiente foi pedido para os participantes escreverem um review enganoso contendo no mínimo 300 caracteres, além de preencherem os campos referenetes a idade, ocupação e gênero.

- Reviews verdadeiros: Os reviews verdadeiros foram manualmente coletados do $\mathrm{Skoob}^{3}$, uma rede social voltada para leitores de livros. Para cada review enganoso foi coletado um review verdadeiro sobre o mesmo livro, contendo a mesma polaridade e um número de palavras semelhante.

Foram coletados 68 reviews enganosos. Após a coleta foi medido o tamanho dos reviews e o número médio de tokens $(\bar{w})$ e caracteres $(\bar{l})$ foi de $\bar{w}=108.94$ tokens $\mathrm{e}$ $\bar{l}=636.76$ caracteres respectivamente. Paralelamente coletamos 68 reviews verdadeiros do Skoob com uma média de $\bar{l}=648.45$ caracteres e $\bar{w}=114.23$ palavras.

2 Link to the form: <https://goo.gl/MfuwRB>

$3<$ https://www.skoob.com.br/> 


\subsection{Resultados e discussão}

Nesta etapa do trabalho utilizamos o Brazilian Portuguese LIWC, bag-of-words e Latent Dirichlet allocation (LDA), ambos apresentados na seção 2.3 para gerar o vetor de atributos utilizado pelos classificadores, SVM e Random Forest. Para a validação dos resultados utilizamos o 10-fold cross-validation calculando acurácia, precisão, revocação e F1-score para a classe dos reviews enganosos.

Para criar o vetor de atributos utilizamos a definição do Vector Space Model (VSM). Inicialmente usamos o VSM para representar o bag of words (apresentado no Capítulo 2. No caso do LIWC: para cada review $r$ foi criado um vetor $v_{r}$ que é representado por $v_{r}=\left[t_{r 0}, t_{r 1}, t_{r 2}, \cdots, t_{r T},\right]$ onde $t_{r} c$ representa a proporção de palavras do tópico $c$ no review $r$.

E para gerar o vetor de atributos utilizando LDA, inicialmente treinamos modelos LDA utilizando como parâmetro número de tópicos $m$ valores entre 2 e 50 tópicos. Após o treinamento dos modelos, para cada review $r$ criamos o vetor de atributos LDA $v_{r}=\left(\beta_{t_{1}}, \beta_{t_{2}}, \ldots, \beta_{t_{m}}\right)$ onde $\beta_{t_{m}}=1$ se o tópico $t_{m}$ aparece no review, caso contrário $\beta_{t_{m}}=0$. Para descobrir um número adequado de tópicos $m$, utilizado como parâmetro, analisamos os resultados utilizando SVM e Random forest e verificamos que os melhores resultados foram obtidos utilizando $m=9$ tópicos.

A Tabela 3 mostra os resultados obtidos utilizando a metodologia mostrada anteriormente. A melhor acurácia foi de $66.7 \%$ utilizando BOW + SVM, mas se considerarmos que precisamos evitar falsos positivos o melhor modelo utilizado foi o $\mathrm{BOW}+$ Random forest que obteve $65.1 \%$ de acurácia, no entanto estes apresentam uma precisão maior, $76.3 \%$.

\begin{tabular}{lcccc}
\hline Features & Accuracy (\%) & Precision (\%) & Recall (\%) & F1 Score (\%) \\
\hline BOW + SVM & $\mathbf{6 6 . 7}$ & 73.5 & 61.9 & 65.8 \\
BOW + Random Forest & 65.1 & $\mathbf{7 6 . 3}$ & 55.7 & 59.9 \\
LIWC + SVM & 52.0 & 51.8 & $\mathbf{9 2 . 9}$ & 66.2 \\
LIWC + Random Forest & 61.4 & 64.9 & 54.1 & 57.9 \\
LDA + SVM & 62.3 & 61.2 & 75.2 & 66.7 \\
LDA + Random Forest & 62.7 & 62.0 & 78.3 & $\mathbf{6 7 . 8}$ \\
\hline
\end{tabular}

Tabela 3 - Resultados obtidos utilizando SVM e Random Forest sobre o corpus de reviews enganosos. 


\subsection{Conclusão}

Apesar do resultado obtido neste experimento parecer bem menor comparado com outros resultados da literatura: e.g., 89.8\% (OTT et al., 2011) e 91.2\% (HERNÁNDEZCASTAÑEDA et al., 2017), suspeitamos que nosso conjunto de dados possui um número muito pequeno de reviews e de uma variedade de livros muito ampla comparado com, por exemplo, o OpSpam (OTT et al., 2011), que é um corpus de reviews enganosos sobre um único tema, hotéis e mesmo assim com um número limitado de hotéis. 


\section{Fake News}

\subsection{Introdução}

Ultimamente, o termo Fake News tem sido muito usado, um neologismo que se refere a notícias ou informações falsas que são apresentadas de uma maneira que parece ser verdadeira. David M. J. Lazer et al. definem Fake News como informações fabricadas que imitam o conteúdo da mídia de notícias no formato de apresentação, mas não no processo de criação ou intenção (LAZER et al., 2018). Um dos casos mais emblemáticos de Fake News na área acadêmica foi o artigo escrito por Andrew Wakefield em 1998 (WAKEFIELD, 1999), publicado na revista The Lancet, apresentando uma ligação entre autismo e a vacina de sarampo, caxumba e rubéola. Como vários outros estudos falharam em replicar estas associações, apresenta-se um sinal claro de falsificação de resultados. Hoje em dia, ainda há consequências deste estudo Fake, onde os pais se recusam a vacinar seus filhos devido ao medo associado a este estudo.

Outras datas mais recentes foram em 2016 quando a palavra Fake News ficou famosa mundialmente devido a dois eventos políticos ( O referendo do Brexit no Reino Unido e as eleições presidencias nos EUA). Estes eventos coincidem com o aumento repentino na pesquisa do termo "fake news" no Google ${ }^{1}$ neste mesmo ano. Por outro lado no Brasil, essa buzzword ficou muito famosa no final de 2018 devido a eleição presidencial.

Atualmente, as Fake News se tornaram um pesadelo devido a grande velocidade de compartilhamento de notícias, as quais viajam rapidamente de celular para celular, de família para famílias. As consequências das Fake News estão por toda parte, desde movimentos anti-vacinas até movimentos políticos (ALLCOTT; GENTZKOW, 2017).

Recentemente, houve um grande esforço para combater as Fake News. Grandes companhias como Facebook, Google, e Bing juntaram forças para criar o "The Trust

$1 \quad<$ http://bit.ly/2og4zvV $>$ 
Project" $"$, que criou o The Trust indicator que ajuda pessoas a diferenciar notícias verdadeiras das Fake News.

O conteúdo das fake news são bastantes diversificados em termo de tópicos, estilo de escrita, e plataformas de mídia. Para ajudar a mitigar os efeitos adversos causados por notícias falsas, é de extrema importância o desenvolvimento de métodos que detectem automaticamente notícias falsas nas mídias sociais (SHU et al., 2017). Apesar da linguagem não ter ligação direta na distinção entre notícias verdadeiras e fake news, as notícias, verdadeiras ou falsas utilizam a linguagem de maneira diferente de modo que pode ser detectados por algoritmos. As maneiras distintas de usar a linguagem para detectar notícias falsas foram exploradas por vários grupos de pesquisa, como (CONROY; RUBIN; CHEN, 2015), (LAZER et al., 2018), (MONTEIRO et al., 2018), (SHU et al., 2017), (PÉREZ-ROSAS et al., 2018) e (JR.; LIM; LING, 2018)

\subsection{Fake.Br Dataset}

Para verificar os atributos linguísticos porposto por Hauch et al. (HAUCH et al., 2015), utilizamos o Fake.Br corpus (MONTEIRO et al., 2018), que é o primeiro corpus anotado sobre fake news em Português. Para construir o corpus os autores coletaram e verificaram 3.600 fake news na internet e coletou, de maneira semi-automática, notícias verdadeiras correspondentes para cada fake news.

Os autores coletaram as fake news de quatro websites: Diario do Brasil, A Folha do Brasil, The Journal Brasil e Top Five TV proveniente de um intervalo de dois anos (01/2016 to $01 / 2018)$. Por outro lado, as notícias verdadeiras foram coletadas utilizando um web crawler nas três maiores agências de notícias do Brasil: G1, Folha de São Paulo e Estadão. Após esta fase, os autores utilizaram a medida de similaridade léxica para escolher as notícias verdadeiras mais similares com as fake news e finalmente fizeram uma verificação manual para garantir que a fake news e a notícia verdadeira abordassem o mesmo assunto.

\subsection{Análise do Fake.Br utilizando char n- gram}

Nesta etapa do trabalho utilizamos o char n-gram para fazer a classificação automática das fake news no corpus Fake.Br. Esta é uma parte do trabalho que ainda está em andamento e será futuramente utilizada num artigo.

$2 \quad<$ https://thetrustproject.org $>$ 


\subsubsection{Character n-gram}

O character n-gram, aqui também chamado de char n-gram) é uma maneira de representar o dados do texto para algoritmos de aprendizagem de máquina. Neste modelo de representação textual cada documento é representado por uma série de n-gram. Um n-gram é uma sequencia contínua de $n$ itens, no caso do character n-gram, os itens são os caracteres do texto. Por exemplo, utilizando o char 3-gram obtemos: "Bom dia"seria representado

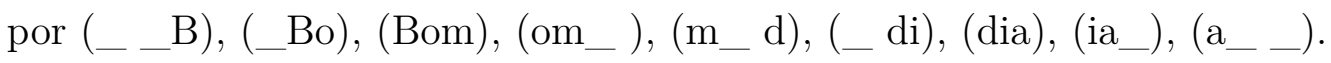

\subsubsection{Metodologia}

Inicialmente todos os caracteres foram convertidos para caracteres minúsculos, números substituídos por "0", links por "URL" e e-mail por "EMAIL". Após este pré-processamento inicial, geramos os char n-gram de todas as notícias variando o $n$ entre 3 e 6 caracteres para verificar qual $n$ que obteve melhor resultado.

Para verificar o melhor $n$, fizemos experimentos utilizando o SVM com kernel linear utilizando os char n-gram, em quatro modelos diferentes: sem remover stopwords e sem aplicar stemming (Figura 3), removendo stopwords (Figura 4), aplicando stemming (Figura 5) e removendo stopwords e aplicando stemming (Figura 6), para verificar o melhor modelo para a classificação.

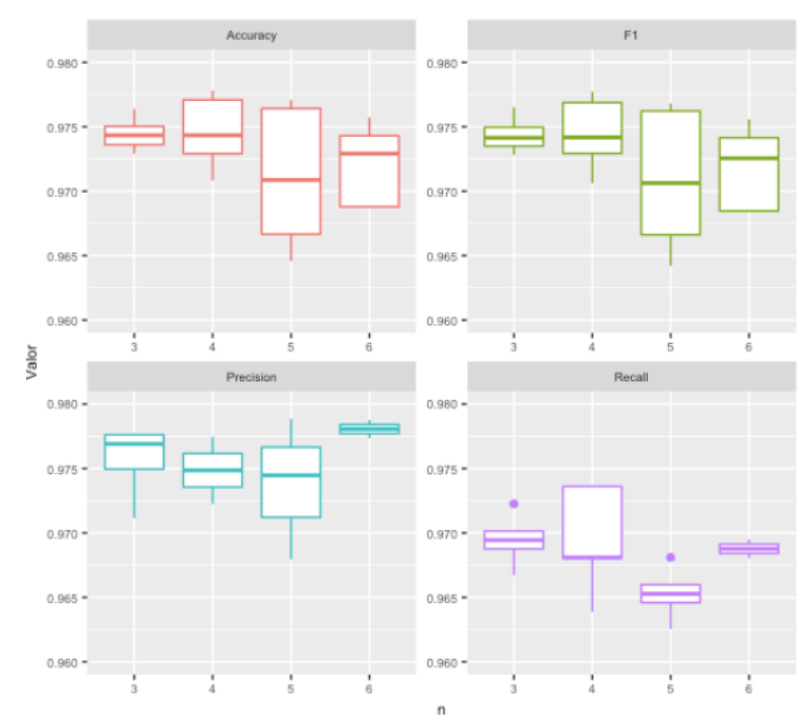

Figura 3 - Boxplot dos resultados obtidos variando o char n-gram.

Fazendo uma analise das Figuras 3, 4, 5 e 6, observamos que apesar dos resultados estarem bem parelhos, os melhores resultados foram obtidos utilizando o char n-gram de 3 caracteres sem utilizar stemming e sem remover stopwords. Para esta análise levamos muito em conta o valor de recall, pois para nos era muito mais importante deixar de classificar 


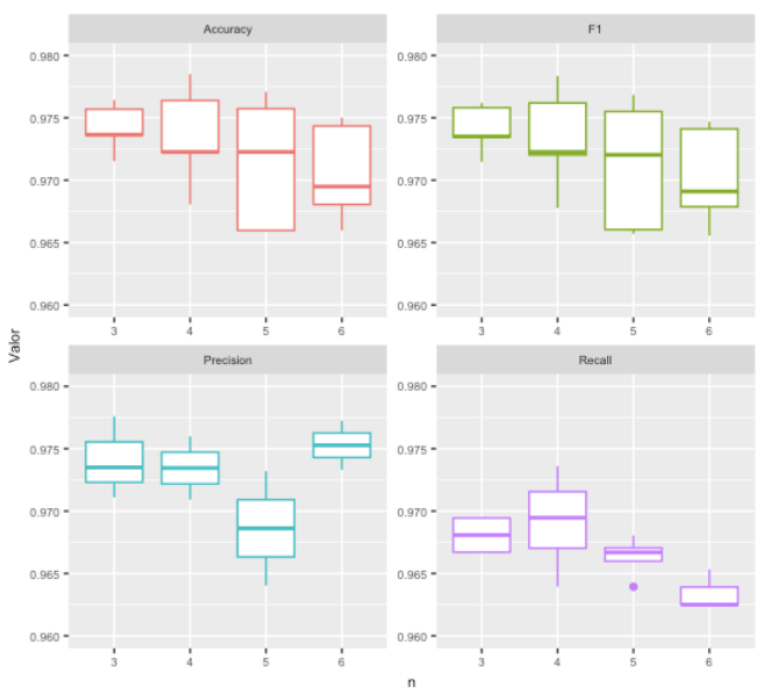

Figura 4 - Boxplot dos resultados obtidos variando o char n-gram removendo stopwords.

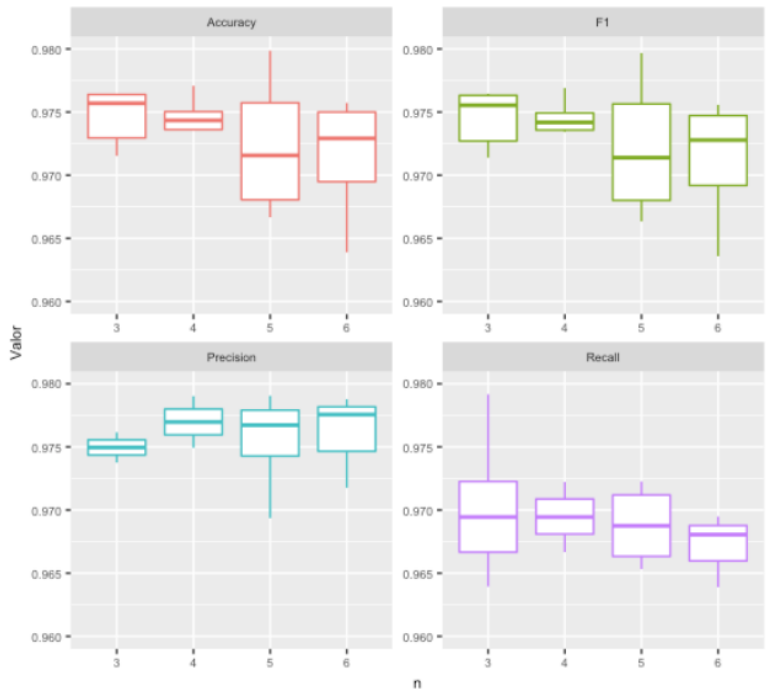

Figura 5 - Boxplot dos resultados obtidos variando o char n-gram aplicando Stemming.

algumas notícias como fake news do que classificar notícias verdadeiras erroneamente como fake news.

Após isto fizemos um ajuste fino nos parâmetros do dicionário gerado pelo char n-gram, no qual fazemos um corte baseado na frequência dos char 3-gram utilizando os parametros no below (figura 7), que remove os itens do dicionário que apareceram em menos de $n$ itens na fase de treinamento e o parâmetro not above (figura 8), que remove os itens do dicionario que aparecem em mais de $n \%$ dos textos da fase de treinamento.

Utilizando os mesmo parâmetros utilizados nas figuras anteriores, fizemos uma análise nas Figuras 7 e 8 buscando escolher os melhores parâmetros para esta tarefa, onde os parâmetros escolhidos para esta tarefa foram: no_below $=8$ e not_above $=0.7$.

Após gerar o vetor de atributos utilizando esta metodologia utilizamos uma série de 


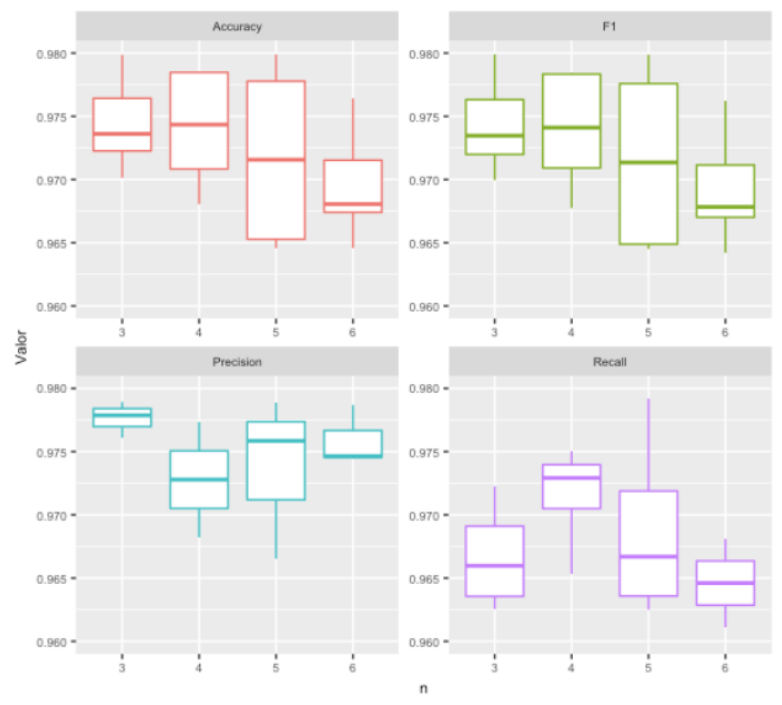

Figura 6 - Boxplot dos resultados obtidos variando o char n-gram removendo stopwords e aplicando stemming.

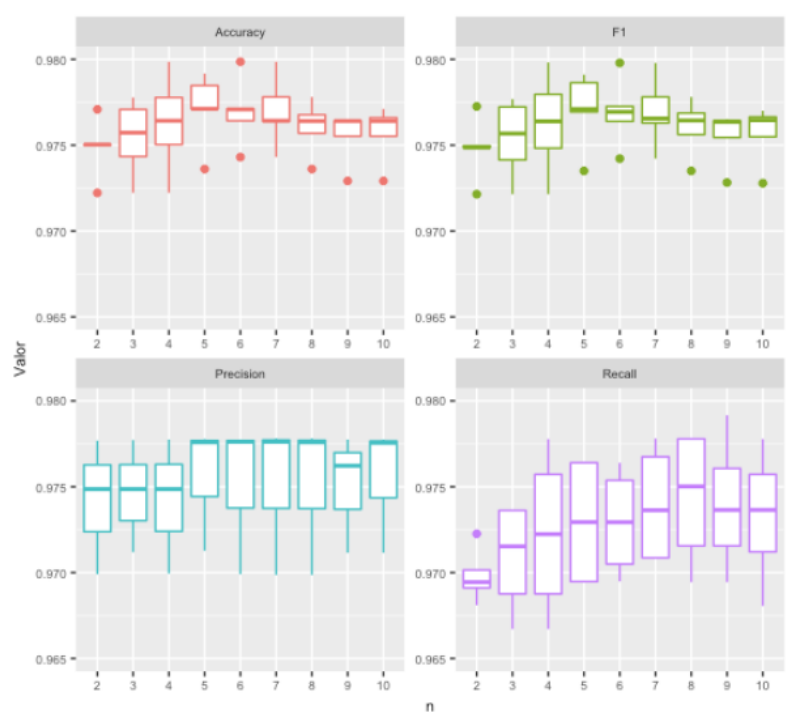

Figura 7 - Boxplot dos resultados obtidos variando o valor do parametro no below.

algoritmos de classificação e para validaçao utilizamos o 5 -fold cross validation calculando acurácia, sensitividade, especificidade, precisão e F-score.

\subsubsection{Resultados}

Na Tabela 4 apresentamos os resultados obtidos utilizando as notícias truncadas. Analisando os resultados observamos que o char n-gram são efetivos para a classificação de fake news. 


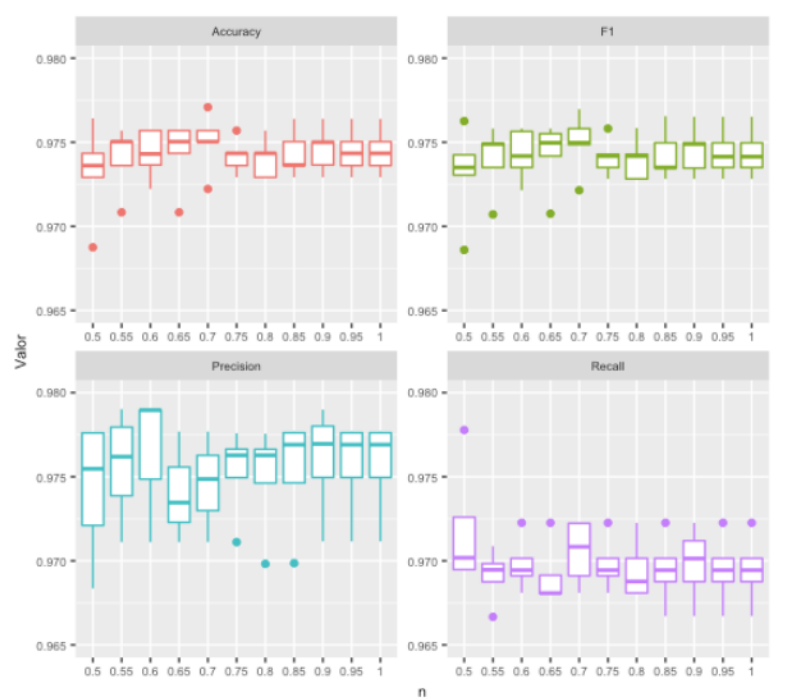

Figura 8 - Boxplot dos resultados obtidos variando o valor do parametro not above.

\begin{tabular}{lrcccc}
\hline Método & Acurácia & Sensitividade & Especificidade & Precisão & F-score \\
\hline Adaboost & 0.9166 & 0.9102 & 0.9228 & 0.9218 & 0.9160 \\
Bagging & 0.8876 & 0.8854 & 0.8898 & 0.8896 & 0.8876 \\
Decision Tree & 0.8052 & 0.8044 & 0.8060 & 0.8056 & 0.8048 \\
Naive Bayes & 0.7318 & 0.6912 & 0.7724 & 0.7540 & 0.7206 \\
Linear SVC & 0.9232 & 0.9136 & 0.9334 & 0.9318 & 0.9226 \\
Logistic Regression & 0.9138 & 0.9056 & 0.9224 & 0.9208 & 0.9130 \\
Random Forest & 0.8902 & 0.8576 & 0.9226 & 0.9176 & 0.8866 \\
\hline
\end{tabular}

Tabela 4 - Resultados obtidos utilizando char 3-gram em textos truncados.

\subsection{Análise do corpus Fake.BR utilizando características psicolinguísticas propos- tas por Hauch et al.}

Nesta etapa do trabalho seguimos os atributos linguísticos apresentados por Hauch et al. (HAUCH et al., 2015) para detectar fake news. Foram testados 76 atributos, inicialmente utilizando a medida de tamanho de efeito Hedges $g$ e em uma segunda parte do trabalho utilizamos algoritmos de aprendizagem de máquina.

\subsubsection{Características psicolinguísticas}

Esta parte do trabalho foi baseada na meta-análise escrita por Hauch et al. (HAUCH et al., 2015), em que os autores investigaram 79 atributos linguísticos extraídos de 44 estudos acadêmicos. Os autores dividiram estas pistas em seis research questions, que são: 
1. Mentirosos sentem uma carga cognitiva maior?

Segundo Hauch et al. (2015) contar uma mentira requer uma carga cognitiva muito maior do que contar uma verdade, pois essa tarefa envolve a execução de uma série de tarefas concorrentes exigindo um grande quantidade de de recursos mentais.

Predições: Dividimos as predições desta Research Question em 3 partes: (a) Length of accounts, (b) Elaboration of accounts e (c) Complexity of accounts. Em (a) esperamos um menor número de palavras, número de sentenças e tamanho médio das sentenças. Em (b) esperamos um menor type-token ratio (Equação 4.1, onde $D W$ corresponde ao número de palavras diferentes e $N W$ ao número de palavras) e tamanho médio de palavras e maior número de palavras com menos de seis caracteres. E em (c) esperamos um número menor de verbos, palavras indicando causa e exclusão.

2. Mentirosos expressam menos certeza do que pessoas verdadeiras?

Seguindo as predições de Hauch et al. (2015) textos enganosos expressam menos certeza do que textos verdadeiros, mas esta predição em particular pode não ser verdadeira no caso de fake news, pois os autores de fake news normalmente tentam escrever suas notícias de maneira mais convincente possível.

Predições: Observando as características das fake news podemos dizer que, contenham mais palavras expressando certeza, menos palavras indicando incerteza e verbos modais.

3. Mentirosos utilizam mais sentimentos negativos?

Segundo Hauch et al. (2015), quando pessoas mentem, elas podem sentir sentimentos de medo e culpa de serem pegos. Até em mentiras cotidianas de pequenas consequências as pessoas se sentem desconfortáveis e assim expressam mais palavras de sentimentos negativos e menos palavras de sentimentos positivos.

a) Mentirosos utilizam mais negação e palavras expressando emoções negativas? Predições: Assim como a research question propõe, predizemos que, textos enganosos possuem mais palavras de negações e mais palavras de emoções negativas em geral: raiva, ansiedade e tristeza.

b) Mentirosos utilizam menos palavras expressando emoções positiva?

Predições: Comparado com as notícias verdadeiras, as fake news contem menos palavras com emoções positivas e emoções afetivas.

c) Mentirosos expressam mais ou menos emoções não especificadas?

Predições: Para esta Research question em particular não temos uma predição.

4. Mentirosos se distanciam dos eventos? 
Nesta research question, Hauch et al. (2015) leva em conta uma consequência da research question 2 e 3. Devido ao sentimento de culpa das pessoas que escrevem textos enganosos, elas tendem a se distanciar dos eventos, evitando se envolver muito na história escrita.

Predições: Em resumo, os autores dos textos enganosos utilizam menos pronomes na primeira pessoa e mais pronomes na terceira pessoa. Assim como o uso mais frequente da voz passiva mostrando sinal de menos envolvimento com o ocorrido.

5. Mentirosos utilizam menos detalhes(sensoriais e contextuais)?

Esta research question é tambem uma consequência da research question anterior. Devido a necessidade dos escritores se distanciar dos eventos, espera-se que eles tambem escrevam menos detalhes sensoriais e contextuais.

Predições: Nesta research question espera-se que os autores utilizem menos palavras sensoriais e perceptivas, menos palavras indicando espaço, tempo, preposições, números, quantidades, modificadores e verbos de movimento.

6. Mentirosos referem-se menos frequentemente a processos cognitivos? Esta research question prevê que escritores de fake news por estar escrevendo sobre uma mentira, ou um fato que não ocorreu, utilizem menos palavras com referência a processos cognitivos.

Predições: Nesta research question espera-se que autores de notícias verdadeiras utilizem mais palavras indicando processos cognitivos e insights.

\section{Miscellaneous}

Esta categoria e composto pelas pistas linguísticas utilizadas nos estudos de Hauch et al. (2015), mas que não tiveram um background teórico ou predições (e.g. processos sociais, esportes).

$$
T T R=\frac{D W}{N W}
$$

Em nosso trabalho, utilizamos 63 dos 79 atributos linguísticos estudados por Hauch et al. (HAUCH et al., 2015). alguns destes atributos linguísticos não foram implementados por falta de recursos de PLN na língua portuguesa. Alguns dos atributos foram realocados para a categoria "miscellaneous", como mostrado por Hauch, estes atributos não se encaixam em nenhuma das research questions.

Para algumas das características apresentadas por Hauch, utilizamos o Brazilian Portuguese LIWC (FILHO; PARDO; ALUÍSIO, 2013), apresentado anteriormente na seção 2.3. Para as categorias restantes utilizamos o Spacy (HONNIBAL; MONTANI, 2017), um módulo open source para processamento do linguagem natural em Python. No 


\begin{aligned} & \hline$g$ Interpretation \\ & \hline 0.01 Very small \\ & 0.2 Small \\ & 0.5 Medium \\ & 0.8 Large \\ & 1.2 Very large \\ & 2 Huge \\ & \hline\end{aligned}

Tabela 5 - Rules of Thumb

Spacy utilizamos o módulo pt_core_news_sm que realiza a rotulação morfo-sintática, part-of-speech tagging, em textos em Português.

\subsubsection{Hedges effect size}

Para verificar a diferença entre as médias destes atributos entre fake news e notícias verdadeiras utilizamos a medida Hedges g. O Hedges g (HEDGES; I., 1985) é um estimador não enviesado da razão de diferença de médias em relação a média ponderada do desvio padrão dos dois grupos, fake news e notícias verdadeiras, e é descrito pela Equação 4.2, onde $M_{1}$ e $M_{2}$ correspondem as médias obtidas nas notícias verdadeiras e fake news respectivamente. Nesta medida $s_{\text {pooled }}^{*}$ corresponde a média ponderada do desvio padrão dos dois grupos, como mostrada pela Equação 4.3, onde $n_{1}$ e $s_{1}$ correspondem ao número de amostras e desvio padrão das notícias verdadeiras e $n_{2}$ e $s_{2}$ correspondem ao número de amostras e desvio padrão das fake news. Neste trabalho, um valor de $g$ positivo é indicativo de notícias verdadeiras e valores negativos de fake news.

$$
\begin{gathered}
g=\frac{M_{1}-M_{2}}{s_{\text {pooled }}^{*}} \\
s_{\text {pooled }}^{*}=\sqrt{\frac{\left(n_{1}-1\right) s_{1}^{2}+\left(n_{2}-1\right) s_{2}^{2}}{n_{1}+n_{2}-2}}
\end{gathered}
$$

Para interpretar os resultados fornecidos pela equação de Hedges nós utilizamos o "Rules of thumb" proposto por Sawilowsky (SAWILOWSKY, 2009), e apresentado na Tabela 5 .

\subsubsection{Resultados}

É importante mencionar que no dataset Fake.Br as notícias verdadeiras são mais longas do que suas fake news correspondentes. Considerando esta informação, fizemos dois experimentos utilizando os atributos mencionados: 1) Nos textos completos, e; 2) Nos 
textos truncados (MONTEIRO et al., 2018), onde para cada par de notícia verdadeira e falsa correspondente o maior texto é truncado para o tamanho do menor. Nós utilizamos os textos completos para fazer uma análise mais detalhada sobre as research questions, e os textos truncados utilizamos para fazer a classificação automática utilizando as características psicolinguísticas mostradas neste estudo.

\section{Análise das Research questions}

Como falamos anteriormente, para a análise das Research questions utilizamos os textos completos para ter uma medida mais realista das fake news em relação as notícias verdadeiras. Nesta parte faremos uma análise dos resultados mostrados nas Tabelas $6 \mathrm{em}$ comparação as predições apresentadas por Hauch et al. (2015).

1. Mentirosos sentem uma carga cognitiva maior?

Como o esperado, autores de fake news utilizam menos palavras em comparação a pessoas que escrevem notícias verdadeiras tendo um maior número de palavras e número de sentenças, outro resultado que foi de acordo com o predito por Hauch et al. (2015) foi um tamanho médio de sentença maior em notícias verdadeiras do que em notícias falsas obtendo um $g=0.73$ que é um valor relativamente alta comparado com os outros resultados obtidos.

Houve também resultados contraditórios as predições propostas como: Type-token ratio que era esperado um hedges positivo, mas o resultado foi muito diferente do esperado $g=-2.72$, mostrando que o o type-token ratio em fake news e bem maior do que em notícias verdadeiras. Mas observando a Equação 4.1 podemos ver que TTR é inversamente proporcional a $N W$, ou seja, como temos uma média de palavras muito maior em notícias verdadeiras a média do type-token ratio é muito menor em notícias verdadeiras.

Observando os resultados obtidos inicialmente podemos dizer que pessoas que escrevem fake news sofrem de uma carga cognitiva maior se observarmos apenas as pistas que não são influenciadas pela quantidade de palavras e quantidade de sentenças. Mas algumas pistas devido a grande diferença entre o número de palavras algumas características (e.g. Type-token ratio, Verbos) ficaram enviesadas devido a grande diferença entre a média de palavras e de sentenças dos dois tipos de notícias.

2. Mentirosos expressam menos certeza do que pessoas verdadeiras?

Os dados apresentados na Tabela 6 mostram que diferente do esperado as fake news apresentam mais palavras indicando incerteza e um número maior de verbos modais mostrando uma leve insegurança dos autores de fake news. Mas por outro lado 
temos também um número maior de palavras indicando certeza, mas com um $g$ um pouco menor.

3. a) Mentirosos utilizam mais negação e palavras expressando emoções negativas? Os resultados apresentados para esta research question foram unanimes, mesmo que pequeno, os resultados demonstraram que pessoas que escrevem fake news em geral utilizam mais palavras de emoções negativas (e.g. Emoções negativas obteve $g=-0.21$ ). Podendo destacar que o sentimento de tristeza que teve um $g=-0.21$.

b) Mentirosos utilizam menos palavras expressando emoções positiva?

Para esta research question não conseguimos obter um resultado muito significativo, pois para emoções positivas obtemos $g=0.09$ que é um valor positivo indicando que possui uma maior ocorrência em notícias verdadeiras, mas este resultado indica uma diferença muito pequena na média dos dois tipos de notícias, o mesmo vale para o resultado obtido para emoções afetivas.

c) Mentirosos expressam mais ou menos emoções não especificadas?

Quanto a emoções não especificadas obtivemos um $g=-0.02$ que é um valor muito baixo, indicando que ambos autores utilizam praticamente a mesma média de emoções não especificadas.

4. Mentirosos se distanciam dos eventos?

Os resultados obtidos em relação a esta research question mostram que tanto os escritores de notícias verdadeiras quanto os de fake news possuem o mesmo estilo de escrita em relação as pistas extraídas para esta research question, podemos observar que $|g|<0.1$ para a maioria das pistas, exceto pelos verbos no passado onde obtivemos $g=-0.14$ indicando que autores de fake news utilizam um pouco mais de verbos no passado.

5. Mentirosos utilizam menos detalhes(sensoriais e contextuais)?

Nesta research question obtivemos resultados muito interessantes, os resultados indicam que os escritores de fake news utilizam mais detalhes sensoriais, ou seja utilizam mais palavras indicando processos perceptivos como visão, tato e audição. Por outro lado escritores de notícias verídicas utilizam mais detalhes contextuais como palavras indicando tempo e espaço, demonstrando o tipo de descrição mais utilizada por ambos os escritores.

Os resultados indicam também que escritores de notícias verdadeiras utilizam mais preposições e modificadores e menos quantificadores indicando que os mesmos escrevem textos mais descritivos.

6. Mentirosos referem-se menos frequentemente a processos cognitivos? 
Como o previsto nesta research question escritores de fake news utilizaram menos palavras indicando processos cognitivos e insights.

M. Miscellaneous Nesta categoria alocamos 21 pistas psicolinguísticas que não foram alocadas a nenhuma research question, dentre estas pistas podemos dizer que escritores de notícias verdadeiras utilizam mais palavras indicando inclusão e utilizam mais sinais de pontuação (pausalidade). E para os escritores de fake news os dois indicadores com maior $g$ foram palavras indicando Amigos e Lazer.

Observando os resultados em gera, podemos concluir que devido a grade diferença no tamanho dos textos tornam algumas características muito relevantes, como o type token ratio. Outro fator importante a ser observado é o coeficiente de variação, que é o valor do desvio padrão dividido pela média, obtidos nas medidas de ambos textos, onde podemos observar um coeficiente de variação muito menor nas notícias verdadeiras, mostrando que as notícias verdadeiras são mais homogêneas. Este resultado pode ter acontecido devido ao fato das notícias verdadeiras terem sido extraídas dos principais sites de notícias do Brasil, onde a notícia passa por várias pessoas antes de chegar ao consumidor final.

\section{Classificação automática}

Para verificar o desempenho destes atributos decidimos utilizar alguns algoritmos de aprendizado de máquina para a classificação. Para isto seguimos o trabalho feito por Monteiro et al. (MONTEIRO et al., 2018), utilizamos o 5-fold cross validation calculando acurácia, precisão, revocação e F-score para cada classe. Analisando os resultados apresentados na Tabela 8, observamos que os atributos psicolinguísticos obtém bons resultados para a tarefa de classificação

\subsection{Hierarchical Attention Network}

Neste capítulo utilizamos o algoritmo de deep learning chamado Hierarchical Attention Network inicialmente apresentado por Yang et al. (2016). Este algoritmo apresentou resultados muitos promissores quando aplicado para análise de sentimentos, além de mostrar as palavras e sentenças mais importantes para a classificação.

\subsubsection{Algoritmo}

A ideia principal por trás deste algoritmo é: "Palavras fazem sentenças e sentenças fazem fazem documentos", ou seja, a intenção do algoritmo é extrair o sentido da sentença a 


\begin{tabular}{|c|c|c|c|c|c|c|}
\hline $\mathrm{RQ}$ & Cue & $g$ & $\bar{x}_{f}$ & $s_{f}$ & $\bar{x}_{t}$ & $s_{t}$ \\
\hline 1 & Quantidade de palavras & 1.86 & 185.92 & 128.12 & 1110.41 & 689.94 \\
\hline 1 & Quantidade de sentenças & 1.52 & 10.30 & 7.21 & 52.34 & 38.42 \\
\hline 1 & Tamanho médio das sentenças & 0.73 & 109.79 & 34.69 & 136.49 & 38.62 \\
\hline 1 & Type-token ratio & -2.72 & 0.6541 & 0.0809 & 0.4518 & 0.0673 \\
\hline 1 & Palavras menores de 6 letras & 0.09 & 0.3804 & 0.0523 & 0.3845 & 0.0326 \\
\hline 1 & Tamanho médio das palavras & 0.13 & 4.8382 & 0.3036 & 4.8715 & 0.2073 \\
\hline 1 & Verbos & -0.50 & 0.1513 & 0.0317 & 0.1376 & 0.0218 \\
\hline 1 & Causa & 0.10 & 0.0302 & 0.0174 & 0.0316 & 0.0097 \\
\hline 1 & Exclusão & 0.01 & 0.0538 & 0.0249 & 0.0540 & 0.0174 \\
\hline 2 & Incerteza & -0.16 & 0.0706 & 0.0283 & 0.0666 & 0.0190 \\
\hline 2 & Verbos Modais & -0.11 & 0.0043 & 0.0064 & 0.0037 & 0.0032 \\
\hline 2 & Certeza & -0.07 & 0.0123 & 0.0109 & 0.0116 & 0.0053 \\
\hline $3(\mathrm{a})$ & Negações & -0.06 & 0.0107 & 0.0114 & 0.0102 & 0.0065 \\
\hline $3(\mathrm{a})$ & Emoções negativas & -0.21 & 0.0383 & 0.0236 & 0.0341 & 0.0144 \\
\hline $3(\mathrm{a})$ & Raiva & -0.13 & 0.0161 & 0.0160 & 0.0144 & 0.0098 \\
\hline $3(\mathrm{a})$ & Ansiedade & -0.13 & 0.0082 & 0.0108 & 0.0070 & 0.0054 \\
\hline $3(\mathrm{a})$ & Tristeza & -0.21 & 0.0092 & 0.0105 & 0.0075 & 0.0050 \\
\hline $3(\mathrm{~b})$ & Emoções positivas & 0.09 & 0.0407 & 0.0214 & 0.0423 & 0.0120 \\
\hline $3(b)$ & Emoções afetivas & -0.09 & 0.0831 & 0.0311 & 0.0809 & 0.0188 \\
\hline $3(\mathrm{c})$ & Emoções não especificadas & -0.02 & 0.2754 & 0.0995 & 0.2741 & 0.0657 \\
\hline 4 & Primeira pessoa do singular & -0.03 & 0.0034 & 0.0090 & 0.0031 & 0.0064 \\
\hline 4 & Primeira pessoa do plural & -0.06 & 0.0009 & 0.0039 & 0.0007 & 0.0016 \\
\hline 4 & Segunda pessoa & 0.01 & 0.0850 & 0.0236 & 0.0851 & 0.0130 \\
\hline 4 & Terceira pessoa do singular & -0.07 & 0.0837 & 0.0255 & 0.0822 & 0.0145 \\
\hline 4 & Terceira pessoa do plural & 0.04 & 0.0171 & 0.0131 & 0.0176 & 0.0074 \\
\hline 4 & Pronomes & -0.06 & 0.0987 & 0.0279 & 0.0974 & 0.0158 \\
\hline 4 & Voz passiva & -0.06 & 0.0099 & 0.0099 & 0.0095 & 0.0054 \\
\hline 4 & Verbos passado & -0.14 & 0.0780 & 0.0315 & 0.0742 & 0.0224 \\
\hline 4 & Verbos presente & -0.03 & 0.3559 & 0.0672 & 0.3543 & 0.0482 \\
\hline 5 & Processos perceptivos & -0.36 & 0.0345 & 0.0212 & 0.0284 & 0.0110 \\
\hline 5 & Visão & -0.08 & 0.0066 & 0.0088 & 0.0060 & 0.0048 \\
\hline 5 & Tato & -0.25 & 0.0116 & 0.0123 & 0.0092 & 0.0050 \\
\hline 5 & Audição & -0.24 & 0.0139 & 0.0130 & 0.0114 & 0.0068 \\
\hline 5 & Tempo & 0.30 & 0.0654 & 0.0253 & 0.0717 & 0.0159 \\
\hline 5 & Espaço & 0.30 & 0.1263 & 0.0346 & 0.1351 & 0.0214 \\
\hline 5 & Preposições & 0.52 & 0.1777 & 0.0370 & 0.1938 & 0.0238 \\
\hline 5 & Números & -0.00 & 0.0170 & 0.0136 & 0.0169 & 0.0077 \\
\hline 5 & Quantificadores & -0.23 & 0.0360 & 0.0192 & 0.0324 & 0.0118 \\
\hline 5 & Modificadores & 0.20 & 0.0199 & 0.0133 & 0.0220 & 0.0077 \\
\hline 5 & Verbos indicando movimento & -0.29 & 0.0567 & 0.0249 & 0.0508 & 0.0138 \\
\hline 6 & Processos cognitivos & 0.22 & 0.3214 & 0.0519 & 0.3314 & 0.0366 \\
\hline 6 & insight & 0.16 & 0.0460 & 0.0217 & 0.0489 & 0.0128 \\
\hline
\end{tabular}

Tabela 6 - Atributos psicolinguísticos extraídos das notícias completas onde: $\mathrm{RQ}=r e$ search question do atributo; Cue = Atributo linguístico; $g=$ Hedges effect size; $\bar{x}_{f}=$ Valor médio dos atributos para fake news; $s_{f}=$ Desvio padrão dos atributos para fake news; $\bar{x}_{t}=$ Valor médio dos atributos para notícias verdadeiras; $s_{f}=$ Desvio padrão dos atributos para notícias verdadeiras. 


\begin{tabular}{rlccccc}
\hline RQ & Cue & \multicolumn{1}{c}{$g$} & $\bar{x}_{f}$ & $s_{f}$ & $\bar{x}_{t}$ & $s_{t}$ \\
\hline $\mathrm{M}$ & Concordar & -0.09 & 0.0036 & 0.0059 & 0.0032 & 0.0027 \\
M & Artigos & -0.07 & 0.0975 & 0.0248 & 0.0961 & 0.0146 \\
M & Inibição & 0.00 & 0.0339 & 0.0192 & 0.0340 & 0.0100 \\
M & Social & -0.17 & 0.1757 & 0.0446 & 0.1692 & 0.0292 \\
M & Amigos & -0.21 & 0.0037 & 0.0064 & 0.0027 & 0.0030 \\
M & Família & -0.03 & 0.0028 & 0.0074 & 0.0026 & 0.0048 \\
M & Humanos & -0.17 & 0.0333 & 0.0202 & 0.0304 & 0.0120 \\
M & Verbos no futuro & -0.12 & 0.0053 & 0.0074 & 0.0045 & 0.0036 \\
M & Inclusão & 0.45 & 0.1399 & 0.0333 & 0.1523 & 0.0207 \\
M & Realização & 0.07 & 0.0227 & 0.0158 & 0.0236 & 0.0084 \\
M & Lazer & -0.26 & 0.0148 & 0.0156 & 0.0116 & 0.0083 \\
M & Emotividade & -0.02 & 0.2754 & 0.0995 & 0.2741 & 0.0657 \\
M & Pausalidade & 0.42 & 2.2786 & 0.6969 & 2.5469 & 0.5687 \\
M & Palavrões & -0.12 & 0.0462 & 0.0222 & 0.0440 & 0.0133 \\
M & Biológico & -0.04 & 0.0417 & 0.0250 & 0.0409 & 0.0148 \\
M & Saúde & -0.03 & 0.0081 & 0.0133 & 0.0078 & 0.0082 \\
M & Sexo & 0.08 & 0.0065 & 0.0083 & 0.0070 & 0.0046 \\
M & Trabalho & 0.13 & 0.0482 & 0.0250 & 0.0509 & 0.0176 \\
M & Casa & 0.02 & 0.0061 & 0.0098 & 0.0063 & 0.0062 \\
M & dinheiro & -0.00 & 0.0207 & 0.0179 & 0.0206 & 0.0112 \\
M & Estados do corpo & -0.13 & 0.0193 & 0.0150 & 0.0178 & 0.0077 \\
\hline
\end{tabular}

Tabela 7 - Atributos psicolinguísticos pertencentes a categoria Miscellaneous em que: RQ $=$ Research question ao qual o atributo linguístico pertence; Cue $=$ Atributo linguístico $g=$ Hedges effect size; $\bar{x}_{f}=$ Valor médio dos atributos para fake news; $s_{f}=$ Desvio padrão dos atributos para fake news; $\bar{x}_{t}=$ Valor médio dos atributos para notícias verdadeiras; $s_{f}=$ Desvio padrão dos atributos para notícias verdadeiras.

\begin{tabular}{lrcccccc}
\hline Method & \multicolumn{3}{c}{ Precision } & \multicolumn{2}{c}{ Recall } & \multicolumn{2}{c}{ F-score } \\
& Accuracy & Fake & True & Fake & True & Fake & True \\
\hline SVM Linear & 0.75 & 0.76 & 0.74 & 0.73 & 0.77 & 0.75 & 0.76 \\
Random forest & 0.75 & 0.75 & 0.75 & 0.74 & 0.75 & 0.75 & 0.75 \\
Logistic regression & 0.75 & 0.76 & 0.74 & 0.73 & 0.76 & 0.74 & 0.75 \\
\hline
\end{tabular}

Tabela 8 - Resultados obtidos utilizando atributos psicolinguísticos em textos truncados. 
partir das palavras e então extrair o sentido do documento a parti das sentenças.

Mas como podemos perceber quando lemos um documento, nem todas as palavras são igualmente importantes para uma sentença, algumas palavras caracterizam mais uma sentença do que outras. Observando este fato, Yang et al. (2016) utilizou uma camada de atenção, no qual o vetor da sentença pode dar mas atenção para palavras mais "importantes" para a sentença. O modelo de atenção consiste em duas partes: RNN (Recurrent neural network) bidirecional e uma rede de atenção.

\section{Gated Recurrent Unit (GRU)}

O Gated Recurrent Unit (GRU) é um mecanismo utilizado em redes neurais recorrentes que são uma classe de redes neurais onde as células formam um grafo direcionado ao longo de uma sequencia temporal, ou seja, no caso da utilização em processamento de linguagem natural uma rede neural recorrente consegue processar e informações obtida levando em conta a sequência das palavras utilizadas.

O GRU utiliza dois mecanismo de gate para decidir o quanto de informação do estado anterior $h_{t-1}$ é utilizada e quanto de informação é atualizada.

Inicialmente é calculado o reset gate (Equação 4.5) que controla o quanto o estado anterior $h_{t-1}$ contribui para o candidate state $\tilde{h}_{t}$. Na Equação 4.5 podemos ver que o reset gate é calculado através de uma função sigmoid (Equação 4.4) onde $W_{r}$ corresponde a um vetor de pesos atribuídos para o vetor de entrada $x_{t}, U_{r}$ corresponde a um vetor de pesos atribuídos para o vetor do estado anterior $h_{t-1}$ e $b_{z}$ corresponde a um vetor de bias. Caso o $r_{t}=0$, então o valor de $h_{t-1}$ será "esquecido".

$$
\begin{gathered}
\sigma(x)=\frac{1}{1+e^{-x}} \\
r_{t}=\sigma\left(W_{r} x_{t}+U_{r} h_{t-1}+b_{r}\right)
\end{gathered}
$$

Paralelamente ao reset gate calculamos o update gate (Equação 4.6) que calcula o quanto de informação do estado anterior $h_{t-1}$ é mantido e o quanto de nova informação é adicionada.

$$
z_{t}=\sigma\left(W_{z} x_{t}+U_{z} h_{t-1}+b_{z}\right)
$$

O candidate state $\tilde{h}_{t}$ representa um novo estado com novas informações que podem ou não ser aproveitadas dependendo do valor do update gate $z_{t}$. Este novo estado é calculado seguindo a Equação 4.8 utilizando a função de tangente hiperbólica (Equação 
4.7) como função de ativação, utilizando valores da entrada $x_{t}$ e dependendo do valor do reset gate $r_{t}$ utilizamos informações do estado anterior $h_{t-1}$.

$$
\begin{gathered}
\tanh (x)=\frac{e^{x}-e^{-x}}{e^{x}+e^{-x}} \\
\tilde{h}_{t}=\tanh \left(W_{h} x_{t}+r_{t} \odot\left(U_{h} h_{t-1}\right)+b_{h}\right)
\end{gathered}
$$

O estado final $h_{t}$ é calculado utilizando $z_{t}$ como parâmetro que controla o quanto $\left(1-z_{t}\right)$ da nova informação $\tilde{h}_{t}$ é utilizada e o quanto $z_{t}$ de informação é utilizado do estado anterior $h_{t-1}$

$$
h_{t}=\left(1-z_{t}\right) \odot \tilde{h}_{t}+z_{t} \odot h_{t-1}
$$

Figura 9 - Hierarchical Attention Network fonte: (YANG et al., 2016)

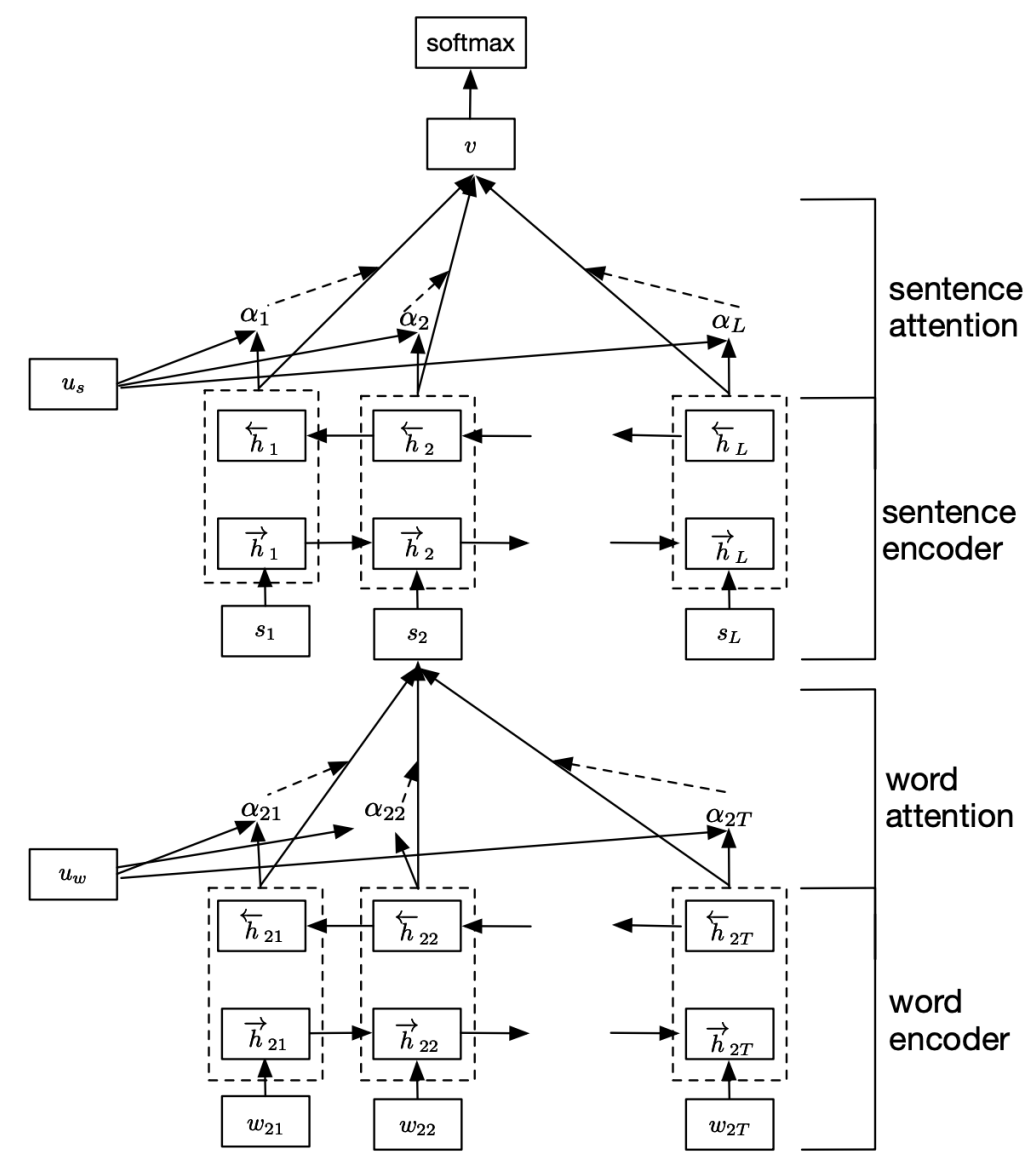

\section{Word encoder}

Dado uma sentença com palavras $w_{i t}, t \in[1, T]$ para $T$ correspondendo ao número máximo de palavras por sentença, transformamos essas palavras em vetores utilizando o GloVe (FI- 
LHO; PARDO; ALUÍSIO, 2013) $x_{i T}$, como o apresentado na Equação 4.10. Esse modelo utiliza um GRU bidirecional, onde palavras são processadas em duas direções, o foward hidden state $\overrightarrow{h_{i t}}$ e o backward hidden state $\overleftarrow{h_{i t}}$, (Equations 4.11 and 4.12 ) desta maneira esta etapa processa a informação da sentença centralizado na palavra $w_{i t}$, como o mostrado na Equação 4.13.

$$
\begin{aligned}
x_{i t} & =W_{e} w_{i t} \\
\overrightarrow{h_{i t}} & =\overrightarrow{\operatorname{GRU}}\left(x_{i t}\right), \\
\overleftarrow{h_{i t}} & =\overleftarrow{\operatorname{GRU}}\left(x_{i t}\right), \\
h_{i t} & =\left[\overrightarrow{h_{i t}}, \overleftarrow{h_{i t}}\right]
\end{aligned}
$$

\section{Word Attention}

O mecanismo de atenção apresentado nesta camada (representado como $\alpha_{i t}$ na Figura 9), calcula pesos para palavras considerando a importância $\alpha_{i t}$ da palavras $w_{i t}$ para a sentença $s_{i}$, em outras palavras, calcula a importância da palavras para a sentença. Em resumo, a camada de atenção é formada por um Multilayer Perceptron (MLP) de uma camada utilizando a função de tangente hiperbólica como função de ativação como o mostrado na Equação 4.14, e então medimos a importância da palavra como a similaridade de $u_{i t}$ com o vetor de contexto $u_{w}$ e calculamos o peso $\alpha_{i t}$ através da função Softmax apresentado pela Equação 4.15. Após calcular o peso de cada palavra $\alpha_{i t}$ calculamos o vetor da sentença $s_{i}$ como a soma ponderada de $h_{i t}$ com seus respectivos pesos $\alpha_{i t}$ (Equação 4.16).

$$
\begin{aligned}
u_{i t} & =\tanh \left(W_{w} h_{i}+b_{w}\right) \\
\alpha_{i t} & =\frac{\exp \left(u_{i t}^{\top} u_{w}\right)}{\sum_{t} \exp \left(u_{i t}^{\top} u_{w}\right)} \\
s_{i} & =\sum_{t} \alpha_{i t} h_{i t}
\end{aligned}
$$

\section{Sentence Attention}

Dado os vetores das sentenças $s_{i}$, assim como na camada Word encoder utilizamos o GRU bidirecional para processar o vetor de sentenças como o mostrado nas Equações 4.17, 4.18 e 4.19 . 


$$
\begin{aligned}
\overrightarrow{h_{i}} & =\overrightarrow{\operatorname{GRU}}\left(s_{i}\right), i \in[1, L] \\
\overleftarrow{h_{i}} & =\overleftarrow{\operatorname{GRU}}\left(s_{i}\right), i \in[L, 1] \\
h_{i} & =\left[\overrightarrow{h_{i}}, \overleftarrow{h_{i}}\right]
\end{aligned}
$$

\section{Sentence Attention}

Um mecanismo de atenção semelhante a camada Word Attention é implementada para das mais importância as sentenças que mais contribuem para a classificação como o mostrado nas Equações 4.20 e 4.21. E a Equação 4.22 apresenta a formação do vetor $v$ através da soma ponderada do vetor de sentenças $s_{i}$ com seus respectivos pesos $\alpha_{i}$

$$
\begin{aligned}
u_{i} & =\tanh \left(W_{s} h_{i}+b_{s}\right) \\
\alpha_{i} & =\frac{\exp \left(u_{i}^{\top} u_{s}\right)}{\sum_{i} \exp \left(u_{i}^{\top} u_{s}\right)} \\
v & =\sum_{i} \alpha_{i} h_{i}
\end{aligned}
$$

\section{Classificação}

Para classificar o documento, utilizamos o vetor do documento $v$ em uma função Softmax como o mostrado na Equação 4.23 , onde $W_{c}$ representa o vetor de pesos e $b_{c}$ representa o vetor de bias.

$$
p=\operatorname{softmax}\left(W_{c} v+b_{c}\right)
$$

Neste trabalho utilizamos a função categorical cross-entropy (Equação 4.24) como loss function. Onde o loss, $L$, é calculado como a entropia de todas as classes $M$, onde $y_{c}$ é o rótulo da classe (notícia verdadeira ou fake news), e $p_{c}$ é o rótulo dado pelo algoritmo.

$$
L=-\sum_{c=1}^{M} y_{c} \log p_{c}
$$

\subsubsection{Configuração do modelo}

Inicialmente, foi feito o preprocessamento do texto, convertendo o texto para letras minúsculas e removendo as stop words (NLTK stop words). Então, dividimos os documentos 
em sentenças utilizando a função sentence tokenizer do NLTK e então utilizando o Keras Tokenizer para dividir as sentenças em tokens.

Na camada de Word embedding, foi utilizado os vetores GloVe treinados por Hartmann et al. (2017). Estes vetores foram treinados em um corpus gigante multi-gênero. Foi utilizado vetores GloVes de diferentes dimensões $(d=100,300,600)$

Na fase de treinamento do modelo, foi utilizado um batch size de 400, utilizando o Keras Adam optmizer como função de loss. O modelo foi treinado utilizando esta configuração durante 100 épocas ou até o valor do loss não diminuir pelo menos em $10^{-3}$ por dez épocas consecutivas.

Para a validação da performance do algoritmo na tarefa de detecção de Fake News, foi utilizado a mesma metodologia usada por Monteiro et al. (2018), executando o algoritmo em dois setups: Utilizando os textos inteiro e utilizando os textos truncados. E para a validação utilizamos o 5-fold cross-validation calculando a precisão, revocação, medida F e acurácia para cada classe.

\subsubsection{Resultados}

Os nossos experimentos foram executados utilizando os textos inteiros e em textos em truncados. A escolha da utilização dos textos truncados foi feito devido a grande diferença entre o número médio de tokens entre as duas classes: notícias verdadeiras $\left(\overline{t_{t}}=1,268.5\right)$ e Fake News $\left(\overline{t_{f}}=216.1\right)$

Na Tabela 9, apresentamos os resultados obtidos utilizando o dataset com textos inteiros. Na primeira coluna mostramos os parâmetros utilizados para o algoritmo, onde GloVe $x$ são os resultados obtidos utilizando GloVe de $x$ dimensões, e SW nós removemos as stop words. Analisando os resultados, podemos observar que todos os resultados obtidos utilizando estes parâmetros em textos inteiros foram semelhantes, mesmo utilizando GloVe de diferente dimensões.

Utilizando este modelo nos textos inteiros, obtemos 97\% de acurácia, mas considerando que a grande diferença entre o numero de tokens entre as duas classes possa facilitar muito a classificação, fizemos os experimentos utilizando os textos truncados.

$\mathrm{Na}$ Tabela 10, apresentamos os resultados utilizando os textos truncados. Onde podemos observar os resultados utilizando HAN foram semelhantes, se não um pouco melhores, do que os resultados apresentados por Monteiro et al. (2018), onde os autores utilizaram principalmente o bag of words para obter uma acurácia de $89 \%$. Uma vantagem desse modelo, que pode fazer uma grande diferença para futuro estudos, é que podemos extrair as palavras e também sentenças que foram mais importantes para a classificação. 


\begin{tabular}{lccccccc}
\hline & \multicolumn{2}{c}{ Precision } & \multicolumn{2}{c}{ Recall } & \multicolumn{2}{c}{ F-score } & Accuracy \\
& Fake & True & Fake & True & Fake & True & \\
\hline GloVe 100 & 0.9655 & 0.9695 & $\mathbf{0 . 9 6 9 4}$ & 0.9653 & 0.9674 & 0.9673 & 0.9674 \\
GloVe 100 SW & 0.9696 & 0.9532 & 0.9522 & 0.9700 & 0.9608 & 0.9615 & 0.9611 \\
GloVe 300 & 0.9666 & 0.9622 & 0.9619 & 0.9667 & 0.9642 & 0.9644 & 0.9643 \\
GloVe 300 SW & 0.9672 & 0.9640 & 0.9639 & 0.9672 & 0.9655 & 0.9656 & 0.9656 \\
GloVe 600 & $\mathbf{0 . 9 7 0 6}$ & $\mathbf{0 . 9 6 9 6}$ & $\mathbf{0 . 9 6 9 4}$ & $\mathbf{0 . 9 7 0 6}$ & $\mathbf{0 . 9 7 0 0}$ & $\mathbf{0 . 9 7 0 0}$ & $\mathbf{0 . 9 7 0 0}$ \\
GloVe 600 SW & 0.9655 & 0.9632 & 0.9631 & 0.9656 & 0.9643 & 0.9643 & 0.9643 \\
\hline
\end{tabular}

Tabela 9 - Resultados obtidos utilizando HAN em textos completos.

\begin{tabular}{lccccccc}
\hline & \multicolumn{2}{c}{ Precision } & \multicolumn{2}{c}{ Recall } & \multicolumn{2}{c}{ F-score } & Accuracy \\
& Fake & True & Fake & True & Fake & True & \\
\hline GloVe 100 & 0.8966 & $\mathbf{0 . 9 0 3 8}$ & $\mathbf{0 . 9 0 4 4}$ & 0.8956 & 0.9004 & 0.8996 & 0.9000 \\
GloVe 100 SW & 0.8801 & 0.8884 & 0.8892 & 0.8769 & 0.8840 & 0.8819 & 0.8831 \\
GloVe 300 & 0.9035 & 0.9018 & 0.9017 & 0.9036 & 0.9026 & 0.9027 & 0.9026 \\
GloVe 300 SW & 0.8808 & 0.8983 & 0.9003 & 0.8764 & 0.8899 & 0.8865 & 0.8883 \\
GloVe 600 & $\mathbf{0 . 9 0 9 4}$ & 0.9026 & 0.9014 & $\mathbf{0 . 9 1 0 3}$ & $\mathbf{0 . 9 0 5 3}$ & $\mathbf{0 . 9 0 6 4}$ & $\mathbf{0 . 9 0 5 8}$ \\
GloVe 600 SW & 0.8999 & 0.8990 & 0.8989 & 0.9000 & 0.8994 & 0.8995 & 0.8994 \\
\hline
\end{tabular}

Tabela 10 - Resultados obtidos utilizando HAN em textos truncados.

\subsection{Conclusão}

Neste capítulo apresentamos três diferentes maneiras de fazer a classificação entre fake news e notícias verdadeiras utilizando o corpus Fake.Br (MONTEIRO et al., 2018). Inicialmente utilizamos a abordagem utilizando char n-gram, características psicolinguísticas e utilizando um algoritmo recente de deep learning conhecido como Hierarchical Attention Network.

Em um trabalho inicial utilizamos o char n-gram para tentar fazer a classificação de fake news utilizando vários valores para $n$ e uma série de algoritmos de aprendizagem de máquina em textos truncados, obtendo o nosso melhor resultado utilizando textos truncados uma acurácia de $92.32 \%$ mostrando a efetividade deste modelo para a classificação das fake news.

Fizemos uma análise das fake news utilizando as características psicolinguísticas apresentadas por Hauch et al. (2015), onde as características foram analisadas dividas por research questions mostrando o impacto de cada uma delas na escolha das palavras, mesmo que pequeno. Fizemos também a classificação automática utilizando algoritmos de aprendizagem de máquina obtendo uma acurácia de $75 \%$ para todos os algoritmos utilizados.

E por ultimo utilizamos o algoritmo de deep learning chamado hierarchical attention network para fazer a classificação automatica do corpus. Apesar do alto custo computacional, não obtivemos o melhor resultado, alcançando uma acurácia de $90.94 \%$ em 
textos truncados. Mas mesmo não obtendo os melhores resultados futuramente podemos utilizar os pesos obtidos nas camadas de atenção para verificar a relevância de algumas palavras para a classificação.

Em geral obtivemos um bom resultado utilizando algoritmos de aprendizagem de máquina, futuramente podemos utilizar a combinação dos atributos estudados neste capitulo para a classificação na tentativa de melhorar os resultados. 



\section{Conclusão}

Inicialmente, devido a carência de um corpus contendo textos enganosos em português, criamos um corpus paralelo contendo review enganosos de livros. Após a criação do corpus fizemos a classificação automática do mesmo utilizando bag of words, LIWC e LDA como atributos juntamente com algoritmos de aprendizagem de máquina. Os resultados obtidos nestes experimentos apesar de não terem sido muito satisfatórios quando comparado com os resultados obtidos em trabalhos semelhantes (e.g. Ott et al. (2011), Hernández-Castañeda et al. (2017)) devemos levar em conta também o pequeno número de review obtidos e também a grande variedade de livros, aumentando muito a variedade de temas dificultando a classificação.

Em 2018 Monteiro et al. (2018) apresentou o corpus Fake.Br onde foi feito a maioria dos experimentos de nosso trabalho. De todos os modelos testados o melhor resultado obtido foi utilizando o char n-gram obtendo $92 \%$ de acurácia utilizando textos truncados, o que é um ótimo resultado. Obtemos resultados interessantes também utilizando os atributos psicolinguísticos propostos por Hauch et al. (2015), além de mostrar diferenças psicolinguísticas entre os dois textos podemos observar que as características são mais homogêneas em notícias verdadeiras, ou seja o desvio padrão das características obtido em notícias verdadeiras são menores. Um dos motivos deste desvio padrão menor é a fonte onde elas foram retiradas, que foi retirado dos principais sites de notícias do Brasil no qual todas as notícias passam por um revisor antes de serem publicadas.

Usamos também um algoritmo de deep learning na tentativa de classificação das fake news na tentativa de obter um resultado melhor e também verificar a influencia das palavras e sentenças para a classificação. Observando os resultados, onde esperávamos um resultado melhor do que os resultados anteriores e o resultados obtidos por Monteiro et al. (2018), obtivemos resultados semelhantes apesar do grande esforço computacional necessário para a execução deste algoritmo. 


\subsection{Contribuições}

O conteúdo criado durante o mestrado gerou três publicações, um student research workshop e dois artigos de conferência.

O conteúdo apresentado no Capítulo 3 gerou o student research workshop (Anexo A) publicado no 13th International Conference on the Computational Processing of Portuguese (PROPOR 2018). E o conteúdo apresentado no Capítulo 4 gerou dois artigos de conferência: 1) Using linguistic cues to detect fakenews on the Brazilian Portuguese parallel corpus Fake.BR (Anexo B) publicado no 12th Brazilian Symposium in Information and Human Language Technology (STIL 2019) e 2) Fake news detection on Fake.Br using hierarchical attention networks (Anexo C) aceito no 14th International Conference on the Computational Processing of Portuguese (PROPOR 2020).

Nosso trabalho contribuiu inicialmente com a criação de um corpus anotado em portugues sobre reviews enganosos de livros, assim como a avaliação de vários modelos já utilizados por outros autores em corpus de opinion spam. Na área de detecção de fake news fizemos uma contribuição considerável verificando o tamanho de efeito de cada pista apresentada pela meta análise publicada por Hauch et al. (2015), mostramos também a efetividade do algoritmo de deep learning hierarchical attention network para a classificação de fake news.

\subsection{Trabalhos futuros}

Como trabalhos futuros, pretende-se: I) Aumentar o número de reviews enganosos de livros para criar um corpus mais robusto, II) Implementar um modelo utilizando as características psicolinguísticas juntamente com o algoritmo de de deep learning hierarchical attention network para verificar a eficácia deste método e III) Pesquisar outros métodos para a detecção de textos intencionalmente enganosos. 


\section{Referências}

ABOUELENIEN, M. et al. Detecting Deceptive Behavior via Integration of Discriminative Features from Multiple Modalities. IEEE Transactions on Information Forensics and Security, v. 12, n. 5, p. 1042-1055, 2017. ISSN 15566013.

ALLCOTT, H.; GENTZKOW, M. Social media and fake news in the 2016 election. Journal of Economic Perspectives, v. 31, n. 2, p. 211-36, 2017.

APPLING, S.; BRISCOE, E.; HUTTO, C. J. Discriminative Models for Predicting Deception Strategies Categories and Subject Descriptors. p. 947-952, 2015.

BIRD, S.; LOPER, E.; KLEIN, E. Natural language processing with python:"o'reilly media inc.". 2009.

BLEI, D. M.; NG, A. Y.; JORDAN, M. I. Latent dirichlet allocation. Journal of Machine Learning Research, v. 3, n. Jan, p. 993-1022, 2003.

BREIMAN, L. Random forests. Machine Learning, v. 45, n. 1, p. 5-32, 2001. ISSN 08856125.

CONROY, N. J. et al. Automatic Deception Detection : Methods for Finding Fake News Automatic Deception Detection : Methods for Finding Fake News. n. OCTOBER, 2015.

CONROY, N. J.; RUBIN, V. L.; CHEN, Y. Automatic deception detection: Methods for finding fake news. Proceedings of the Association for Information Science and Technology, Wiley Online Library, v. 52, n. 1, p. 1-4, 2015.

CRAWFORD, M. et al. Survey of review spam detection using machine learning techniques. Journal of Big Data, Journal of Big Data, v. 2, n. 1, p. 23, 2015. ISSN 2196-1115. Disponível em: <http://www.journalofbigdata.com/content/2/1/23>.

DePaulo, B. M. et al. Cues to deception. Psychological Bulletin, v. 129, n. 1, p. 74-118, 2003. ISSN 0033-2909. Disponível em: < http://doi.apa.org/getdoi.cfm?doi=10.1037/ 0033-2909.129.1.74>.

DIXIT, S.; AGRAWAL, A. J. Survey on review spam detection. International Journal of Computer \& Communication Technology, v. 4, n. 2, p. 68-72, 2013.

FALEIROS, T. P.; LOPES, A. A. Modelos probabilísticos de tópicos-desvendando o Latent Dirichlet Allocation. p. 2662-2673, 2016.

FILHO, P. P. B.; PARDO, T. A. S.; ALUÍSIO, S. M. An evaluation of the brazilian portuguese liwc dictionary for sentiment analysis. In: Proceedings of the 9th Brazilian Symposium in Information and Human Language Technology. [S.l.: s.n.], 2013. 
FORNACIARI, T.; POESIO, M. Identifying fake Amazon reviews as learning from crowds. 14th Conference of the European Chapter of the Association for Computational Linguistics 2014, EACL 2014, p. 279-287, 2014. Disponível em: < http: //www.scopus.com/inward/record.url?eid=2-s2.0-84905717452 $\{\&\}$ partnerID=tZOtx $>$.

GOKHMAN, S. et al. In Search of a Gold Standard in Studies of Deception. Computational Linguistics, n. 2003, p. 23-30, 2012.

HANCOCK, J. T. et al. On Lying and Being Lied To: A Linguistic Analysis of Deception in Computer-Mediated Communication. Discourse Processes, v. 45, n. 1, p. 1-23, 2007. ISSN 0163-853X. Disponível em: <http://www.tandfonline.com/doi/abs/10.1080/ 01638530701739181>.

HANCOCK, J. T. et al. Deception and Design : The Impact of Communication Technology on Lying Behavior. Media, v. 6, n. 1, p. 129-134, 2004. Disponível em: $<$ http://portal.acm.org/citation.cfm?doid=985692.985709>.

HARTMANN, N. et al. Portuguese word embeddings: Evaluating on word analogies and natural language tasks. arXiv preprint arXiv:1708.06025, 2017.

HAUCH, V. et al. Are Computers Effective Lie Detectors? A Meta-Analysis of Linguistic Cues to Deception. Personality and Social Psychology Review, v. 19, n. 4, p. 307-342, 2015. ISSN 1088-8683. Disponível em: <http://journals.sagepub.com/doi/10.1177/ $1088868314556539>$.

HEDGES, L. V.; I., O. Statistical methods for meta-analysis. San Diego, CA: Academic Press, 1985.

HERNÁNDEZ-CASTAÑEDA, Á. et al. Cross-domain deception detection using support vector networks. Soft Computing, Springer Berlin Heidelberg, v. 21, n. 3, p. 585-595, 2017. ISSN 1432-7643. Disponível em: <http://link.springer.com/10.1007/s00500-016-2409-2>.

HEYDARI, A. et al. Detection of review spam: A survey. Expert Systems with Applications, Elsevier Ltd, v. 42, n. 7, p. 3634-3642, 2015. ISSN 09574174. Disponível em: <http://dx.doi.org/10.1016/j.eswa.2014.12.029>.

HO, S. M. et al. Real or spiel? A decision tree approach for automated detection of deceptive language-action cues. Proceedings of the Annual Hawaii International Conference on System Sciences, v. 2016-March, p. 3706-3715, 2016. ISSN 15301605.

HONNIBAL, M.; MONTANI, I. spaCy 2: Natural language understanding with Bloom embeddings, convolutional neural networks and incremental parsing. To appear, 2017.

JINDAL, N.; LIU, B. Opinion spam and analysis. Proceedings of the international conference on web search and web data mining 2008, p. 219-230, 2008. Disponível em: $<$ http://portal.acm.org/citation.cfm?doid=1341531.1341560>.

JR., E. C. T.; LIM, Z. W.; LING, R. Defining "fake news": A typology of scholarly definitions. Digital Journalism, Taylor \& Francis, v. 6, n. 2, p. 137-153, 2018.

JURAFSKY, D.; MARTIN, J. H. Speech and Language Processing. [s.n.], 2008. 950 p. ISBN 978-0131873216. Disponível em: <http://www.cs.colorado.edu/\{ $\}$ martin/slp.ht $>$. 
LAZER, D. M. et al. The science of fake news. Science, American Association for the Advancement of Science, v. 359, n. 6380, p. 1094-1096, 2018.

LORENA, A. C.; CARVAlHO, A. C. P. L. F. D. C. Uma Introdução às Support Vector Machines. RITA, Revista de informática Teórica e Aplicada, 2007.

MIHALCEA, R.; STRAPPARAVA, C. The Lie Detector: Explorations in the Automatic Recognition of Deceptive Language. Proceedings of the ACL-IJCNLP 2009 Conference Short Papers, n. August, p. 309-312, 2009. Disponível em: $<$ http://portal.acm.org/citation.cfm?id=1667679>.

MONTEIRO, R. A. et al. Contributions to the study of fake news in portuguese: New corpus and automatic detection results. In: Computational Processing of the Portuguese Language. [S.l.]: Springer International Publishing, 2018. p. 324-334. ISBN 978-3-319-99722-3.

MURPHY, K. P. Naïve Bayes classifiers Generative classifiers. Bernoulli, v. 4701, n. October, p. 1-8, 2006. ISSN 03029743. Disponível em: <http://www.springerlink.com/ index/0060368703612735.pdf $>$.

OTT, M.; CARDIE, C.; HANCOCK, J. T. Negative Deceptive Opinion Spam. Naacl-Hlt, n. June, p. 497-501, 2013.

OTT, M. et al. Finding Deceptive Opinion Spam by Any Stretch of the Imagination. Proceedings of the 49th Annua Meeting of the Association for Computational Linguistics, p. 11, 2011. Disponível em: <http://arxiv.org/abs/1107.4557>.

PÉREZ-ROSAS, V. et al. Automatic Detection of Fake News. In: Proceedings of the 27th International Conference on Computational Linguistics. Santa Fe, New Mexico, USA: Association for Computational Linguistics, 2018. p. 3391-3401. Disponível em: $<$ https://www.aclweb.org/anthology/C18-1287>.

RUBIN, V. L. et al. Towards News Verification : Deception Detection Methods for News Discourse. v. 2015, p. 1-5, 2015.

SAWILOWSKY, S. S. New Effect Size Rules of Thumb. Journal of Modern Applied Statistical Methods, v. 8, n. 2, p. 26, 2009.

SHU, K. et al. Fake news detection on social media: A data mining perspective. ACM SIGKDD Explorations Newsletter, ACM, v. 19, n. 1, p. 22-36, 2017.

TAUSCZIK, Y. R.; PENNEBAKER, J. W. The Psychological Meaning of Words: LIWC and Computerized Text Analysis Methods. Journal of Language and Social Psychology, v. 29, n. 1, p. 24-54, 2010. ISSN 0261-927X. Disponível em: $<$ http://journals.sagepub.com/doi/10.1177/0261927X09351676>.

TSIKERDEKIS, M.; ZEADALLY, S. Online Deception in Social Media. Communications of the ACM, v. 57, n. 9, p. 72-80, 2014. ISSN 00010782.

VAPNIK, V. N. An overview of statistical learning theory. IEEE transactions on neural networks, IEEE, v. 10, n. 5, p. 988-999, 1999.

WAKEFIELD, A. J. MMR vaccination and autism. The Lancet, Elsevier, v. 354, n. 9182, p. 949-950, 1999. 
YANG, Z. et al. Hierarchical attention networks for document classification. In:

Proceedings of the 2016 Conference of the North American Chapter of the Association for Computational Linguistics: Human Language Technologies. [S.l.: s.n.], 2016. p. 1480-1489.

ZHOU, L.; SHI, Y.; ZHANG, D. A statistical language modeling approach to online deception detection. IEEE Transactions on Knowledge and Data Engineering, v. 20, n. 8, p. 1077-1081, 2008. ISSN 10414347.

ZHOU, L.; ZHANG, D. Following Linguistic Footprints: Automatic Deception Detection in Online Communication. Communications of the ACM, v. 51, p. 119-122, 2008. ISSN 00010782. Disponível em: < http://search.ebscohost.com/login.aspx?direct=true\&db= aph\&AN=34141240\&site $=$ ehost-live $>$. 
Anexos 



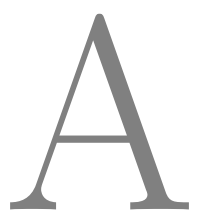

Finding deceptive book reviews in Brazilian Portuguese 


\title{
Finding deceptive book reviews in Brazilian Portuguese
}

\author{
Emerson Yoshiaki Okano ${ }^{[0000-0001-8684-9301]}$, Verônica Letícia Oyan de \\ Moraes, Mateus Tarcinalli Machado[0000-0003-1848-3082], and Evandro Eduardo \\ Seron Ruiz ${ }^{[0000-0002-7434-897 X]}$ \\ Departamento de Computação e Matemática - FFCLRP, \\ Universidade de São Paulo, \\ Avenida dos Bandeirantes, 3900 - Monte Alegre, CEP 14040-901, Ribeirão Preto-SP , \\ Brasil. \\ \{okano700, veronica.leticia.moraes, mateusmachado, evandro\}@usp.br
}

\begin{abstract}
User-generated reviews of products and services play a major role in customer's decisions. However, such reviews are not always reliable. The widespread use of deceptive reviews has increased interest in datasets and methods to recognize these malicious opinions automatically. In this study, we first assembled and analyzed deceptive book reviews in Brazilian Portuguese. We collected 68 truthful and 68 deceptive reviews, which amounted to 136 reviews. We experimented with the categorization of deceptive reviews by means of Support Vector Machines (SVM) and Random forest with features such as bag-of-words (BOW), the Linguistic Inquiry and Word Count dictionary, and Latent Dirichlet Allocation. Our strategy based on SVM with BOW provided an accuracy of $66.7 \%$. We believe that an increment in the number of reviews may lead to even higher accuracy values.
\end{abstract}

Keywords: Deceptive reviews · Opinion spam - Review spam.

\section{Introduction}

The web constitutes a vast source of textual information, but not every piece of information presented therein is reliable. Some users might use the web to spread false information with the purpose of manipulating and deceiving other users [10]. Most online shoppers rely on product reviews before buying a product. Unfortunately, there are people who take advantage of this behavior and create false product reviews, which are usually designated "deceptive review". These kinds of reviews are known to be an effective component of the buyer's final decision. In this paper, we consider "deceptive reviews" as a form of "opinion spam".

Deceptive reviews, which Dixit et al. [4] also called opinion spam, have been categorized into three groups: (1) Untruthful reviews, which deliberately mislead readers or opinion mining systems; (2) Reviews on brands, which contain comments that are only concerned with the brand, or the seller of the product, and fail to review the product itself; and (3) Non-reviews, which present 
either unrelated text or advertisements. The present work is mainly concerned with untruthful reviews, which are challenging for humans to detect according to Ott et al. [10]. These authors also demonstrated that distinguishing between real reviews and spam is a harder task for man than for computers.

Although the English language relies on some annotated datasets; that is, actual corpora consisting of deceptive reviews and real ones (e.g., Yelp [8], OpSpam [10]), that are useful to test algorithms for deceptive opinion categorization, the Portuguese language lacks similar datasets and methods associated with it. Looking at this opportunity, our primary aim is to assemble a dataset of deceptive book reviews in the Portuguese language for use in deceptive opinion spam detection.

There are many ways to construct an annotated corpus of deceptive texts, as studied by Gokhman et al. [5]. They divided corpora construction approaches into: a) Traditional approach, b) Non-gold standard, and c) Crowdsourcing approach. They also commented about the advantages and limitations of each approach and discussed about methods to handle plagiarism. The corpus building process used in the present work follows some steps proposed by Gokhman.

Due to the lack of a dataset of deceptive review or opinion spam for the Portuguese language, our proposal for a Master dissertation project is to create an annotated parallel corpus consisting of deceptive book reviews and real ones, both written in Brazilian Portuguese, and to use machine learning methods to detect deceptive texts correctly. Hereafter, the paper is organized as follows: Section 2 presents state-of-the-art technology to detect opinion spam. Section 3 the dataset and the developed methodology is described. Section 4 depicts the methods used herein. Section 5 presents the results and discusses them.

\section{Related work}

Annotated opinion review corpora are essential to measure how methods perform during opinion spam detection. The English language relies on several corpora for this purpose, but no corpus of this sort exists for the Portuguese language. Gokhman et al. [5] described two traditional strategies to create a deceptive text corpus: a) a sanctioned corpus, in which the experimenter instructs individuals to lie, or not to lie, in their reviews, and; b) an unsanctioned corpus, in which the participant lies on his or her own accord.

Ott et al. [10] created the Opinion Spam dataset, called OpSpam, which is the first gold standard labeled dataset for opinion spam. To create this dataset, deceptive opinions were obtained through the Amazon Mechanical Turk crowdsourcing service, whereas the truthful opinions were mined from TripAdvisor. In their paper, Ott et al. [10] showed that it is hard for humans to detect deceptive opinion spam, but when these authors applied Support Vector Machines (SVM) and Linguistic Inquiry and Word Count (LIWC)+bigram, they achieved 89.8\% accuracy. Later, Hauch et al. [6] analyzed several studies about deceptive text identification. Their work compared several linguistic cues, most of which were psycholinguistic features that can be analyzed with the aid of the LIWC 
tool [12]. This research showed some linguistic cues that help to differentiate between liars and truth-tellers.

In a recent paper, Castañeda et al. [7] showed the performance of the Latent Dirichlet Allocation (LDA) with word-space model, which outperformed almost all the other models in terms of deceptive text detection. Castañeda et al. obtained an average accuracy of $86 \%$ in one-domain setting, $75 \%$ in mixed-domain setting, and 52 to $64 \%$ in the cross-domain setting.

\section{Dataset: assembling a deceptive book review corpus}

While truthful opinions are ubiquitous online, deceptive opinions are difficult to obtain without resorting to heuristic methods [10]. In this section, we describe how we assembled a deceptive book review dataset.

Deceptive reviews: We assembled this dataset by using the sanctioned approach of Gokhman et al. [5] , which proposes requesting deceptive book reviews. We explicitly asked the participants to lie about a book while writing a review about it. We assembled a deceptive corpus by using Google Forms ${ }^{1}$. We used this service to collect deceptive opinion spam at no cost. We asked the participants to write a book review consisting of 300 characters or more about any of their preferred books and to disclose their age, occupation, and gender. These features might be useful for future research.

Up to this moment, we have gathered 68 deceptive reviews. We have measured the length of the reviews and their average number of words $(\bar{w})$ and characters $(\bar{l})$ is $\bar{w}=108.94$ words and $\bar{l}=636.76$ characters, respectively.

Truthful reviews: We manually mined these reviews from $\mathrm{Skoob}^{2}$, a fourmillion-user Brazilian book-oriented social network. For each deceptive review, we retrieved a truthful one about the same book; the pairs of truthful and deceptive reviews showed the same sentiment polarity and had a similar number of words.

We gathered 68 truthful reviews with $\bar{l}=648.45$ characters and $\bar{w}=114.23$ words, on average.

\section{Automated approaches for review spam detection}

We tested two basic automated approaches for deceptive review detection, both of which were based on a trained classifier, SVM, and Random Forest. We also used the ten-fold cross-validation approach as a validation process. The features supplied to these classifiers are shown below.

\section{Linguistic Inquiry and Word Count (LIWC) and Brazilian LIWC}

Linguistic Inquiry and Word Count (LIWC) [11] is a word-counting-based tool that relies on manually labeled word groups. LIWC classifies words into emotional, cognitive, and structural component categories. Although LIWC was not

\footnotetext{
${ }^{1}$ Link to the form: https://goo.gl/MfuwRB

${ }^{2}$ https://www.skoob.com.br/
} 
specifically designed to assess deception, Newman et al. [9] and Hauch et al. [6] showed that its psycholinguistic features may be used to detect deceptive texts.

In 2013, Balage et al. [1] translated the English lexical LIWC into Portuguese. They also evaluated it for a Brazilian Portuguese sentiment classification task. Since Newman and Hauch used original LIWC to detect deceptive texts in English, we will use the Brazilian Portuguese LIWC to detect deceptive reviews.

\section{Text categorization}

Following Ott's nomenclature [10], we use frequency n-gram-based features for the text categorization approach. These features allow us to model the context of both the content and the word. In this work, we employ the bag-of-words (unigram) approach as feature to analyze lowercased and unstemmed texts.

\section{Latent Dirichlet Allocation (LDA)}

Latent Dirichlet Allocation (LDA) [2] is a probabilistic generative model for discrete data collections, such as text collection, that represents documents as a mix of topics. In this case, a topic is defined as a set of words that often occur in semantically related documents. As a result, each document can combine different topics. In contrast to LIWC, which is a manually annotated lexicon, LDA generates the topics automatically, so it does not depend on language.

\section{Support Vector Machine}

One of the classifiers used here is the support vector machine (SVM), which has been continuously used for text classification $[8,10]$. The SVM algorithm finds a high-dimensional separating hyperplane between two data groups. As a simple example, consider a linear SVM, as described in Equation 1, that can be used as a classifier, where $\boldsymbol{w}$ is the weight vector, $b$ is the bias learned, and $\boldsymbol{x}$ is the feature vector.

$$
\hat{y}=\operatorname{sgn}(\boldsymbol{w} \cdot \boldsymbol{x}+b) \quad \operatorname{sgn}(x)= \begin{cases}1 & \text { if } x>0 \\ -1 & \text { if } x<0\end{cases}
$$

\section{Random Forest}

Random forest is an ensemble machine learning algorithm; more specifically, it is a combination of tree predictors, so that each tree depends on the values of an independently sampled random vector with the same distribution for all trees in the forest [3]. In other words, random forest is a set of decision trees that are used to determine the final outcome. 


\section{$5 \quad$ Results and Discussion}

LIWC, bag-of-words (BOW), and LDA have been used to construct the features used by both classifiers, SVM and Random forests. We evaluated the performance of both classifiers by stratified 10-fold cross-validation, which divides the corpus into ten folds while maintaining the same proportion of deceptive and truthful texts. We used nine of the folds to train the classifiers and the remaining fold to test it.

The LIWC features were formed from a list of all its $n$ topics $t_{0}, t_{1}, \ldots, t_{n}$. Consider $R$ as the set of all reviews. For each review $r_{i}$, deceptive or not, we used the proportion of words of each topic $t_{n}$ was used to assemble an LIWC vector $v_{r_{i}}=\left(\alpha_{1} t_{1}, \alpha_{2} t_{2}, \ldots, \alpha_{n} t_{n}\right)$, where $\alpha_{n}$ is the proportion of words in a topic $t_{n}$ in the review $r_{i}$.

To generate LDA features, we first trained an LDA model by using all the reviews $r_{i}$ containing between 2 and 50 topics $(m)$ as parameters. Then, for each trained model, we created a list containing all the $m$ topics $t_{0}, t_{1}, \ldots, t_{m}$ trained. For each review $r_{i}$ we assembled an LDA vector $v_{r_{i}}=\left(\beta_{1} t_{1}, \beta_{2} t_{2}, \ldots, \beta_{m} t_{m}\right)$ where $\beta_{m}=1$ if the topic $t_{m}$ appeared in the review $r_{i}$, or $\beta_{m}=0$. The SVM and the Random forest classifier were applied to all $m=[2,50]$ topics. The best results for LDA were obtained for $m=9$ topics.

Table 1. Automated classifier performance for SVM and Random Forest based on stratified 10-fold cross-validation experiments, using F1-score over deceptive reviews.

\begin{tabular}{|l|c|c|c|c|}
\hline Features & Accuracy (\%) & Precision (\%) & Recall (\%) & F1 Score (\%) \\
\hline BOW + SVM & $\mathbf{6 6 . 7}$ & 73.5 & 61.9 & 65.8 \\
BOW + Random Forest & 65.1 & $\mathbf{7 6 . 3}$ & 55.7 & 59.9 \\
LIWC + SVM & 52.0 & 51.8 & $\mathbf{9 2 . 9}$ & 66.2 \\
LIWC + Random Forest & 61.4 & 64.9 & 54.1 & 57.9 \\
LDA + SVM & 62.3 & 61.2 & 75.2 & 66.7 \\
LDA + Random Forest & 62.7 & 62.0 & 78.3 & $\mathbf{6 7 . 8}$ \\
\hline
\end{tabular}

Table 1 lists some results. The best overall accuracy, $66.7 \%$, was obtained by considering the vector of features formed from the BOW+SVM. If we avoid false positives, the BOW + Random Forest approach gets almost the same accuracy, $65.1 \%$, but with higher precision $76.3 \%$. Although these results seem discouraging if compared to others; e.g., $89.8 \%$ [10] and $91.2 \%$ [7], we suspect our corpus still has a small number of reviews, which came from many different books. We intend to collect more deceptive and truthful reviews and to continue to research other features and approaches to detect deceptive reviews.

\section{Acknowledgement}

This research was supported by the São Paulo State Research Foundation (FAPESP). 


\section{References}

1. Balage Filho, P.P.P., Aluísio, S., Pardo, T.T.: An Evaluation of the Brazilian Portuguese LIWC Dictionary for Sentiment Analysis. 9th Brazilian Symposium in Information and Human Language Technology - STIL pp. 215-219 (2013)

2. Blei, D.M., Edu, B.B., Ng, A.Y., Edu, A.S., Jordan, M.I., Edu, J.B.: Latent Dirichlet Allocation. Journal of Machine Learning Research 3, 993-1022 (2000)

3. Breiman, L.: Random forests. Machine Learning 45(1), 5-32 (2001)

4. Dixit, S., Agrawal, A.J.: Survey on review spam detection. International Journal of Computer \& Communication Technology 4(2), 68-72 (2013)

5. Gokhman, S., Hancock, J., Prabhu, P., Ott, M., Cardie, C.: In Search of a Gold Standard in Studies of Deception. Computational Linguistics pp. 23-30 (2012)

6. Hauch, V., Blandón-Gitlin, I., Masip, J., Sporer, S.L.: Are Computers Effective Lie Detectors? A Meta-Analysis of Linguistic Cues to Deception. Personality and Social Psychology Review 19(4), 307-342 (2015)

7. Hernández-Castañeda, Á., Calvo, H., Gelbukh, A., Flores, J.J.G.: Cross-domain deception detection using support vector networks. Soft Computing 21(3), 585$595(2017)$

8. Mukherjee, A., Venkataraman, V., Liu, B., Glance, N.: What Yelp Fake Review Filter Might Be Doing? Seventh International AAAI Conference on Weblogs and Social Media pp. 409-418 (2013)

9. Newman, M.L., Pennebaker, J.W., Berry, D.S., Richards, J.M.: Personality and Social Psychology Bulletin. Society for Personality and Social Psychology, Inc. 29(5), 665-675 (2003)

10. Ott, M., Choi, Y., Cardie, C., Hancock, J.T.: Finding Deceptive Opinion Spam by Any Stretch of the Imagination. Proceedings of the 49th Annual Meeting of the Association for Computational Linguistics p. 11 (2011)

11. Pennebaker, J.W., Chung, C.K., Ireland, M., Gonzales, A., Booth, R.J.: The Development and Psychometric Properties of LIWC2007 The University of Texas at Austin. Development 1(2), 1-22 (2007)

12. Tausczik, Y.R., Pennebaker, J.W.: The Psychological Meaning of Words: LIWC and Computerized Text Analysis Methods. Journal of Language and Social Psychology 29(1), 24-54 (2010) 

Using linguistic cues to detect fake news on the Brazilian Portuguese parallel corpus Fake.BR 


\title{
Using linguistic cues to detect fake news on the Brazilian Portuguese parallel corpus Fake.BR
}

\author{
Emerson Yoshiaki Okano ${ }^{1}$, Evandro Eduardo Seron Ruiz ${ }^{1}$ \\ ${ }^{1}$ Departamento de Computação e Matemática, FFCLRP, Universidade de São Paulo \\ Av. Bandeirantes, 3900, Monte Alegre. CEP: 14040-901, Ribeirão Preto - SP \\ Brazil \\ \{okano700, evandro\}@usp.br
}

\begin{abstract}
Fake news has become a significant concern because of their ease propagation through social networks. The use of natural language processing (NLP) tasks to detect fake news has soared lately as human beings usually fail on this task. The purpose of the current work is to detect fake news written in Portuguese using psycholinguistic cues. We tested 76 linguistic characteristics on the Fake.Br parallel corpus. Results show an accuracy of $75 \%$ using three distinctive machine learning methods.
\end{abstract}

Resumo. As Fake news se tornaram uma grande preocupação devido a fácil propagação através de redes sociais. O uso de processamento de linguagem natural (PLN) para detectar fake news tem aumentado ultimamente, já que os seres humanos normalmente falham nessa tarefa. O objetivo deste trabalho é detectar fake news escritas em Português usando características psicolinguísticas. Nós testamos 76 características psicolinguisticas no corpus paralelo Fake.Br. Os resultados apresentados foram uma acurácia de $75 \%$ utilizando três métodos de aprendizado de máquina distintos.

\section{Introduction}

The web constitutes a vast source of textual information, but not every piece of information presented therein is reliable. Some users might use the web to spread false information to manipulate and deceive other users [Ott et al. 2011]. One kind of false information is what is called fake news, which is everywhere nowadays. The term fake news is usually referred to false news published with the aim of achieving some advantage by spreading false information. Fake news has increased as the ease of access to the web and also due to the growing use of web-based social networks.

The term 'fake news' became popular with key political events including the Brexit vote in the UK and the US presidential election in 2016. In Brazil, it became well known just after the 2018 presidential elections.

Recently, there have been many national and international efforts to reduce the effects of false or misleading news or even reveal the hidden truth in fake news creating web pages known as fact pages checking. Two well known Brazilian initiatives are: Me engana que eu posto ${ }^{1}$, and Agência Lupa ${ }^{2}$. Following this same line, companies such as

\footnotetext{
${ }^{1}$ https://veja.abril.com.br/blog/me-engana-que-eu-posto/

${ }^{2}$ https://piaui.folha.uol.com.br/lupa/
} 
Google, Facebook, and Bing have joined forces to create The Trust Project ${ }^{3}$, which consists of an international consortium of news organizations that are collaborating intending to create journalism standards to produce a more reliable press.

Some authors used psycholinguistic features trying to classify deceptive texts [Hauch et al. 2015, Ott et al. 2011]. Myle Ott [Ott et al. 2011] achieved an accuracy of $89.8 \%$ using Linguistic Inquiry and Word Count (LIWC)+Bigrams and SVM as classifier. Valerie Hauch [Hauch et al. 2015] presented a meta-analysis of linguistic cues to deception, showing which cues are better to detect deception in texts.

In our paper, we followed the cues showed through a meta-analysis conducted by Hauch [Hauch et al. 2015] to detect fake news. As a novelty, we used these cues as features for some classic machine learning classification algorithms. We also used the effect size measure to show which cue has more influence in fake and true news characterization.

We organized this paper as follows: Section 2 presents state-of-the-art detection of opinion spam. Section 3 shows the methodology used by Hauch [Hauch et al. 2015] and in this work. Section 4 presents the dataset used in this work. Section 5 we show the results obtained with our methodology. Section 6 presents the conclusion of this paper.

\section{Related work}

Recent work in linguists has explored textual indicators to be implemented by computer systems to discriminate between truths and lies.

Myle Ott and colleagues [Ott et al. 2011] pioneered in the study of psycholinguistic cues using NLP. They used three different automated approaches to detect deceptive opinion spam, which are: a) Frequency distribution of the part-of-speech (POS) tags (word categories) in a text which are often dependent on the genre of the text; b) Psycholinguistic deception detection, whereas the Linguistic Inquiry and Word Count (LIWC) [Tausczik and Pennebaker 2010] is a powerful tool for textual analysis and it has been used to analyze deception [Hancock et al. 2007], and, finally; c) Text categorization approaches, like bag of words and n-gram. They consider n-grams as effective features for deception detection, allowing to model both content and context of the message. Ott and co-workers also achieved their best results using bigrams + LIWC features with SVM as classifier [Ott et al. 2011].

According to [Rubin et al. 2015a] there are three types of Fake news: a) Serious Fabrications: that are the fraudulent news, fake contents with the purpose of manipulating and deceiving the public; b) Large-Scale Hoaxes: rumors that are not confirmed that attempts to deceive audiences masquerade as news; c) Humorous Fakes: that are the one humorous content like News satire sites and news parody. According to Rubin, hybrid methods combining linguist cues and machine learning are very promising approaches. In an earlier article [Rubin et al. 2015b], Rubin and co-workers applied a vector space model to cluster the news by discourse feature similarity, achieving $63 \%$ accuracy.

Mihalcea and collegaues [Pérez-Rosas et al. 2018] also worked on fake news detection. They introduced two novel datasets for the task of fake news detection and con-

\footnotetext{
${ }^{3}$ https://thetrustproject.org/
} 
ducted a set of learning experiments to build fake news detectors. They achieved accuracies of up to $76 \%$.

\section{Methods}

We based our work on a meta-analysis proposed by Hauch and coworkers [Hauch et al. 2015], where the authors investigated 79 linguistic cues compiled from 44 other academic studies. Hauch et al. allocated these cues to six research questions:

1. Do liars experience greater cognitive load?

2. Are liars less certain than truth-tellers?

3. (a) Do liars use more negations and negative emotion words?

(b) Do liars use fewer positive emotion words?

(c) Do liars express more or less unspecified emotion words?

4. Do liars distance themselves more from events?

5. Do liars use fewer (sensory and contextual) details?

6. Do liars refer less often to cognitive processes?

In our work, we investigated 76 linguistic cues from the 79 cues studied by [Hauch et al. 2015]. Three of them (Writing errors, Pleasantness and unpleasantness, and redundancy) have not been implemented due to a lack of resources. Some linguistic cues were originally allocated to a miscellaneous (M) category, as Hauch showed they did not fit into any research question. To extract some of the linguistic cues, we mainly used the Brazilian Portuguese LIWC [Balage Filho et al. 2013], which is a translation of the original one [Tausczik and Pennebaker 2010]. LIWC is an English lexicon that classifies words in psycholinguistic and linguistic categories.

For some linguistic cues (e.g. pausality and verb quantity) we used Spacy $^{4}$ [Honnibal and Montani 2017], a free, open-source library for Natural Language Processing in Python. From Spacy we used the pt_core_news_sm module which performs part-of-speech tagging in Portuguese texts.

We conducted our experiments in two steps: 1) first, we calculated the effect size measure $g$ of each of the 76 linguistic cues. The top ten $g$ measures were selected and shown for the fake and the real news; then; 2) We applied a machine learning approach to classify fake news from the real ones.

The $g$ proposed by Hedges's [Hedges and I. 1985] is an unbiased estimator of the standardized mean difference. Equation 1 presents $g$ where $M_{1}$ is the mean value of the attribute in true news and $M_{2}$ is the mean value of the attribute in fake news. Otherwise, $s_{\text {pooled }}^{*}$, the weighted average of standard deviations for the two groups, is given by Equation 2, where $n_{1}, s_{1}$ and $n_{2}, s_{2}$ are the numbers and standard deviation of true and fake news respectively. Positive $g$ indicates truth and negatives $g$ are indicative of fake news.

$$
g=\frac{M_{1}-M_{2}}{s_{\text {pooled }}^{*}}
$$

\footnotetext{
${ }^{4}$ https: / / spacy.io/
} 


$$
s_{\text {pooled }}^{*}=\sqrt{\frac{\left(n_{1}-1\right) s_{1}^{2}+\left(n_{2}-1\right) s_{2}^{2}}{n_{1}+n_{2}-2}}
$$

To interpret the hedges $g$ effect size we used the "Rules of thumb" adopted by Sawilowsky [Sawilowsky 2009], here presented in Table 1.

\begin{aligned} & \hline$g$ Interpretation \\ & \hline 0.01 Very small \\ & 0.2 Small \\ & 0.5 Medium \\ & 0.8 Large \\ & 1.2 Very large \\ & 2 Huge \\ & \hline\end{aligned}

Table 1. Rules of Thumb.

Next, after calculating the values for all the 76 cues for the 7,200 news (equally divided into two classes, fake and real), we applied three machine learning algorithms to perform an automatic classification of news, they were: Support Vector Machine, SVM, with a linear kernel; Random Forest, and; Logistic regression. We used the 5-fold crossvalidation procedure and computed accuracy, precision, recall, and F-score for each class.

\section{Fake.Br corpus}

In our work, to process the linguistic cues proposed by [Hauch et al. 2015], we used the Fake.Br corpus [Monteiro et al. 2018], which is the first corpus containing fake news in Brazilian Portuguese. To build this corpus, the authors manually collected and checked 3,600 fake news on the internet and semi-automatically looked for corresponding true news for each fake one. The authors collected all the fake news from an interval of two years (from 01/2016 to 01/2018) from 4 websites: Diário do Brasil, A Folha do Brasil, The Journal Brasil and Top Five TV. On the other hand, the authors collected the true news using a web crawler in three major news agencies in Brazil: G1, Folha de São Paulo and Estadão. After the web crawler, the authors used a lexical similarity measure to choose the most similar to the fake news collected. They also performed a manual verification to guarantee that the fake news and true news are subject related.

\section{Results}

It is worth mentioning that often the real news has longer sentences than their corresponding fake news. In this section, we will present the results obtained using the cues, mentioned in Section 3, in the Fake.Br corpus, using two different approaches: 1) using the full text, and; 2) using the truncated text [Monteiro et al. 2018], wherein each fake-true pair, the longer text is truncated to the size (number of tokens) of the shorter text.

\subsection{Full text}

First, we performed our experiments using the full text of the corpus. In Table 2 we presented the top 10 positive and negative hedges, $g$. For this experiment the average absolute effect size was $\bar{g}=0.31$ and an its standard deviation was $s_{g}=0.53$. 
Analyzing the results obtained in Table 2, we observed that we obtained five cues from the research question 5 showing that, in general, fake news uses more sensory and contextual details than the ones used for true news. These results contradict the results obtained from [Hauch et al. 2015] for the same research question (5). We observed some cues have higher values of $g$ than others (e.g., Type token ration, number of verbs). After analyzing these clues, we realized that they are determined by news containing a large number of tokens when compared to their equivalent fake version. Generally, the number of tokens per news is not a well-balanced feature. The mean number of tokens for fake news is 185.92 while for true news is 1110.40 .

\begin{tabular}{rlccccc}
\hline RQ & Cue & $g$ & $\bar{x}_{f}$ & $s_{f}$ & $\bar{x}_{t}$ & $s_{t}$ \\
\hline 1 & Type-token ratio & -2.72 & 0.6541 & 0.0809 & 0.4518 & 0.0673 \\
5 & Perceptual processes & -0.36 & 0.0345 & 0.0212 & 0.0284 & 0.0110 \\
5 & Motion & -0.29 & 0.0567 & 0.0249 & 0.0508 & 0.0138 \\
$\mathrm{M}$ & Leisure & -0.26 & 0.0148 & 0.0156 & 0.0116 & 0.0083 \\
5 & Feel & -0.25 & 0.0116 & 0.0123 & 0.0092 & 0.0050 \\
5 & Hear & -0.24 & 0.0139 & 0.0130 & 0.0114 & 0.0068 \\
$\mathrm{M}$ & Auxiliary verbs & -0.23 & 0.0505 & 0.0219 & 0.0462 & 0.0145 \\
5 & Quantifiers & -0.23 & 0.0360 & 0.0192 & 0.0324 & 0.0118 \\
$3(\mathrm{a})$ & Negative emotion & -0.21 & 0.0383 & 0.0236 & 0.0341 & 0.0144 \\
$3(\mathrm{a})$ & Sadness & -0.21 & 0.0092 & 0.0105 & 0.0075 & 0.0050 \\
\hline $\mathrm{M}$ & Pausality & 0.50 & 2.5329 & 0.7777 & 2.8804 & 0.6166 \\
5 & Prepositions & 0.52 & 0.1777 & 0.0370 & 0.1938 & 0.0238 \\
1 & Average sentence length & 0.73 & 109.7863 & 34.6865 & 136.4863 & 38.6173 \\
2 & Modal verbs & 1.01 & 0.8456 & 1.4014 & 4.2136 & 4.4796 \\
4 & Passive voice verbs & 1.48 & 1.8303 & 1.9291 & 10.0644 & 7.6385 \\
1 & Sentence quantity & 1.52 & 10.3014 & 7.2094 & 52.3358 & 38.4219 \\
1 & Verb & 1.71 & 28.0431 & 20.0078 & 156.0322 & 104.2411 \\
4 & Past tense verbs & 1.74 & 14.3539 & 10.4727 & 81.2647 & 53.2152 \\
1 & Length & 1.86 & 185.9167 & 128.1151 & 1110.3969 & 689.9285 \\
4 & Present tense verbs & 1.91 & 62.4783 & 41.6863 & 378.0736 & 229.7128 \\
\hline
\end{tabular}

Table 2. Top 10 negative and positives linguistic cues extracted from full text where: $R Q=$ Research question for the cue it belongs to (where $M$ stands for miscellaneous category); Cue = Linguistic cue itself; $g=$ Hedges effect size; $\bar{x}_{f}=$ mean value for fake news; $s_{f}=$ standard deviation for fake news; $\bar{x}_{t}=$ mean value in true news; $s_{t}=$ standard deviation in true news.

\subsection{Truncated Texts}

Using the truncated text, we obtained more reliable results, as shown in Table 3 . In Table 4 we described the best cues shown in Table 3 for better understanding. In this experiment we obtained a lower average absolute effect size $\bar{g}=0.24$ and a lower standard deviation $s_{g}=0.17$ than using full text.

In Table 3, we observed that we have seven cues from the overall twenty presented in the same table that belongs to the miscellaneous category, which is a category that contains cues that could not be allocated in any of the research questions. From these 
cues, we can notice that social-related words and auxiliary verbs are frequently used in fake news having $g=-0.6$, a medium effect size.

Another interesting cue is the Average sentence length that has a hedges $g=0.74$ showing that true news has more words per sentence than fake news. This feature may be interpreted as true news are being more elaborate texts than fake news.

\begin{tabular}{rlccccc}
\hline RQ & Cue & $g$ & $\bar{x}_{f}$ & $s_{f}$ & $\bar{x}_{t}$ & $s_{t}$ \\
\hline 4 & Pronouns & -0.65 & 0.1420 & 0.0374 & 0.1180 & 0.0367 \\
$\mathrm{M}$ & Social processes & -0.60 & 0.1756 & 0.0446 & 0.1497 & 0.0420 \\
$\mathrm{M}$ & Auxiliary verbs & -0.60 & 0.0504 & 0.0219 & 0.0381 & 0.0193 \\
4 & Impersonal pronouns & -0.55 & 0.1189 & 0.0330 & 0.1009 & 0.0328 \\
5 & Personal pronouns & -0.54 & 0.0986 & 0.0279 & 0.0838 & 0.0270 \\
2 & Tentative & -0.50 & 0.0705 & 0.0283 & 0.0567 & 0.0268 \\
5 & Quantifiers & -0.49 & 0.0360 & 0.0192 & 0.0270 & 0.0180 \\
$\mathrm{M}$ & Articles & -0.48 & 0.0974 & 0.0248 & 0.0854 & 0.0257 \\
$\mathrm{M}$ & Function words & -0.46 & 0.4271 & 0.0488 & 0.4046 & 0.0496 \\
4 & 3rd pers. singular & -0.44 & 0.0836 & 0.0256 & 0.0724 & 0.0250 \\
\hline $\mathrm{M}$ & Work & 0.14 & 0.0482 & 0.0250 & 0.0519 & 0.0284 \\
$\mathrm{M}$ & Pausality & 0.15 & 2.5595 & 0.7788 & 2.6823 & 0.8972 \\
5 & Time & 0.17 & 0.0654 & 0.0253 & 0.0701 & 0.0302 \\
4 & Present tense verbs & 0.20 & 61.6869 & 38.6631 & 69.4503 & 40.0187 \\
5 & Space & 0.21 & 0.1264 & 0.0346 & 0.1339 & 0.0363 \\
1 & Six-letter words & 0.24 & 0.3800 & 0.0523 & 0.3923 & 0.0492 \\
1 & Average word length & 0.27 & 4.8338 & 0.3025 & 4.9149 & 0.2965 \\
$\mathrm{M}$ & Inclusive & 0.27 & 0.1400 & 0.0336 & 0.1494 & 0.0357 \\
5 & Prepositions & 0.63 & 0.1777 & 0.0371 & 0.2001 & 0.0338 \\
1 & Average sentence length & 0.74 & 109.7942 & 34.7963 & 140.1972 & 46.4695 \\
\hline
\end{tabular}

Table 3. Top 10 negative and positives linguistic cues extracted from truncated text where: $\mathbf{R Q}=$ Research question for the cue it belongs to (where $\mathbf{M}$ stands for miscellaneous category); Cue $=$ Linguistic cue itself; $g=$ Hedges effect size; $\bar{x}_{f}=$ mean value for fake news; $s_{f}=$ standard deviation for fake news; $\bar{x}_{t}=$ mean value in true news; $s_{t}=$ standard deviation in true news.

\subsection{Classification task}

To verify the performance of the psycholinguistic features in automatic classifying of fake news, we used some classic machine learning algorithms with the psycholinguistic features extracted from the truncated texts.

Following the work of [Monteiro et al. 2018], we used the 5-fold cross-validation computing accuracy, precision, recall, and F-score for each class. In table 5, we show the results obtained using the psycholinguistic features. Observing the classification results presented in Table 5, we achieved good results taking into account that we used only psycholinguistic features.

\section{Conclusion}

In our paper, we presented the research questions, and the clues previously showed by Hauch and colleagues [Hauch et al. 2015] and, as they did in their work, we used the 


\begin{tabular}{rll}
\hline RQ & Cue & Operational definition \\
\hline 4 & Pronouns & \% of pronouns (e.g., eu, nós) \\
M & Social processes & \% of words that express social processes \\
M & Auxiliary verbs & (e.g., falar, amigos) \\
4 & Impersonal pronouns & \% of impersonal pronouns (e.g., aquele, esse) \\
5 & Personal pronouns & \% of personal pronouns (e.g., eu, ele) \\
2 & Tentative & \% of tentative words (e.g., talvez, possivelmente) \\
5 & Quantifiers & \% of quantifiers words (e.g., alguns, muitos) \\
M & Articles & \% of articles \\
M & Function words & \% of articles, pronouns, verbs \\
4 & 3rd pers. singular & \% of 3rd pers. singular (e.g., ele, ela) \\
\hline M & Work & \%of words that express job issues \\
& & (e.g., chefe, carreira) \\
M & Pausality & Total number of punctuation marks divided \\
5 & Time & by total number of sentences \\
4 & Present tense verbs & \% of temporal words (e.g., tarde, hora) \\
5 & Space & \% of spatial words (e.g., fora, espaçoso) \\
1 & Six-letter words & \% of words that are longer than six letters \\
1 & Average word length & Total number of letters divided by the \\
M & Inclusive & total number of words \\
5 & Prepositions & \% of inclusive words (e.g., dentro, junto) \\
1 & Average sentence length & \% of prepositions \\
& & Total number of words divided by \\
& &
\end{tabular}

Table 4. Top 10 negative and positives linguistic cues extracted from truncated text where: $R Q=$ Research question for the cue it belongs to (where $M$ stands for miscellaneous category); Cue = Linguistic cue itself; Operational definition $=$ describes the cue itself.

Hedges $g$ effect size to measure the influence of each clue in true and fake news.

To verify the performance of these clues under automatic classification, we applied three machine learning algorithms to classify the news as fake or real. The classification results presented in Table 5 are comparable to [Monteiro et al. 2018] except when they used the bag of words approach. Although [Pérez-Rosas et al. 2018] used two different corpora, they also achieved similar results for fake news detection. Fake news generally uses words commonly adopted at the time the news are created. Given this fact, gazetteer approaches able to discern between fake and true news would have to rely on this timedependent dictionaries. One can say that the method used in this paper relies on a steady, reliable, and less dependent time dictionary, the LIWC.

\section{Acknowledgements}

This research was supported by Fundação de Amparo à Pesquisa do Estado de São Paulo (FAPESP), process number: 2018/03129-8. We would like to include a special note of thanks to Dr. Tiago Agostinho de Almeida for helpful comments on this project. 


\begin{tabular}{lrcccccc}
\hline Method & \multicolumn{3}{c}{ Precision } & \multicolumn{2}{c}{ Recall } & \multicolumn{3}{c}{ F-score } \\
& Accuracy & Fake & True & Fake & True & Fake & True \\
\hline SVM Linear & 0.75 & 0.76 & 0.74 & 0.73 & 0.77 & 0.75 & 0.76 \\
Random forest & 0.75 & 0.75 & 0.75 & 0.74 & 0.75 & 0.75 & 0.75 \\
Logistic regression & 0.75 & 0.76 & 0.74 & 0.73 & 0.76 & 0.74 & 0.75 \\
\hline
\end{tabular}

Table 5. Results obtained using machine learning in truncated texts.

\section{References}

Balage Filho, P. P., Aluísio, S. M., and Pardo, T. A. S. (2013). An Evaluation of the Brazilian Portuguese LIWC Dictionary for Sentiment Analysis. 9th Brazilian Symposium in Information and Human Language Technology - STIL, pages 215-219.

Hancock, J. T., Curry, L. E., Goorha, S., and Woodworth, M. (2007). On lying and being lied to: A linguistic analysis of deception in computer-mediated communication. Discourse Processes, 45(1):1-23.

Hauch, V., Blandón-Gitlin, I., Masip, J., and Sporer, S. L. (2015). Are Computers Effective Lie Detectors? A Meta-Analysis of Linguistic Cues to Deception. Personality and Social Psychology Review, 19(4):307-342.

Hedges, L. V. and I., O. (1985). Statistical methods for meta-analysis. Academic Press, San Diego, CA.

Honnibal, M. and Montani, I. (2017). spaCy 2: Natural language understanding with Bloom embeddings, convolutional neural networks and incremental parsing. To appear.

Monteiro, R. A., Santos, R. L. S., Pardo, T. A. S., de Almeida, T. A., Ruiz, E. E. S., and Vale, O. A. (2018). Contributions to the study of fake news in portuguese: New corpus and automatic detection results. In Computational Processing of the Portuguese Language, pages 324-334. Springer International Publishing.

Ott, M., Choi, Y., Cardie, C., and Hancock, J. T. (2011). Finding deceptive opinion spam by any stretch of the imagination. In Proceedings of the 49th Annual Meeting of the Association for Computational Linguistics: Human Language Technologies, volume 1, pages 309-319. Association for Computational Linguistics.

Pérez-Rosas, V., Kleinberg, B., Lefevre, A., and Mihalcea, R. (2018). Automatic detection of fake news. In Proceedings of the 27th International Conference on Computational Linguistics, pages 3391-3401, Santa Fe, New Mexico, USA. Association for Computational Linguistics.

Rubin, V. L., Chen, Y., and Conroy, N. J. (2015a). Deception detection for news: Three types of fakes. Proceedings of the Association for Information Science and Technology, 52(1):1-4.

Rubin, V. L., Conroy, N. J., and Chen, Y. (2015b). Towards news verification: Deception detection methods for news discourse. In Hawaii International Conference on System Sciences.

Sawilowsky, S. S. (2009). New Effect Size Rules of Thumb. Journal of Modern Applied Statistical Methods, 8(2):26. 
Tausczik, Y. R. and Pennebaker, J. W. (2010). The psychological meaning of words: LIWC and computerized text analysis methods. Journal of Language and Social Psychology, 29(1):24-54. 
Fake news detection on Fake.Br using hierarchical attention networks 


\title{
Fake news detection on Fake.Br using hierarchical attention networks
}

\author{
Emerson Yoshiaki Okano ${ }^{10000-0001-8684-9301]}$, Zebin Liu ${ }^{2[0000-0002-5018-8705]}$, \\ Donghong Ji2[0000-0001-9613-5927], and Evandro Eduardo Seron \\ Ruiz $^{1[0000-0002-7434-897 X]}$ \\ 1 Departamento de Computação e Matemática - FFCLRP, \\ Universidade de São Paulo, \\ Avenida dos Bandeirantes, 3900 - Monte Alegre, CEP 14040-901, Ribeirão Preto, SP, \\ Brasil. \\ \{okano700, evandro\}@usp.br \\ 2 School of Cyber Science and Engineering, \\ Wuhan University, \\ \#299, Bayi Road, Wuchang District, Wuhan, 430072, China. \\ dhji@whu.edu.cn, 1579670185@qq.com
}

\begin{abstract}
Automatic fake news detection is a challenging problem in natural language processing, and contributions in this field may induce immense social impacts. This article examines the use of Hierarchical Attention Network (HAN) as a method for automatic fake news detection. We evaluate the proposed models in the Brazilian Portuguese fake news parallel corpus Fake.Br using its original full text, and also in the truncated version. We run the HAN varying the size of word embedding from 100 to 600 , and by maintaining and removing the stop words. This method achieved an accuracy of $97 \%$ for full texts using the word embedding size of 600 from GloVe. However, when comparing running this method for truncated texts, this method presents similar results (90\% accuracy) to the baseline established by the simple machine learning methods presented in the original presentation work of the Fake.Br (89\% accuracy). Overall, keeping or removing stop words and varying the size of the word embeddings also shows a negligible advantage.
\end{abstract}

Keywords: Hierarchical attention networks · Deep learning - Text classification · Fake news.

\section{Introduction}

Lately, the term fake news is of frequent use, a neologism, referring either as misinformation, fabricated news, or false information presented in a way that it may seem factually accurate. David M. J. Lazer and colleagues define "fake news" as fabricated information that mimics news media content in form but not in organizational process or intent [8]. One of the most notorious cases of fake news in science is related to the 1998 paper by Andrew Wakefield [17], published in The Lancet, reporting a link between autism and the measles, mumps, and rubella 
(MMR) vaccine. As numerous other studies fail to replicate these associations, this is a real sign of falsified data in the original study. Nowadays, Wakefield's dangerous claims are still causing direct harm to children whose parents insist not to vaccinate them due to the alleged lifelong conditions imposed by autism.

Another landmark was in 2016 when the word fake news became very famous due to two decisive political events (the Brexit referendum in the UK and the presidential election in the US). These events coincide with the rise of searches for the term "fake news" in Google ${ }^{3}$ in this year. On another hand, this buzzword becomes famous in Brazil in 2018 due to the presidential election.

Presently this fake news nightmare turned to be the high-speed phenomena carrying this fabricated news from cellphones to cellphones, from families to families. The consequences of fake news are everywhere, from the anti-vaccine movement to politics [1].

Recently there has been much effort to fight against fake news. Companies such as Facebook, Google, and Bing have joined forces to create "The Trust Project" ${ }^{4}$, that created the Trust indicator which helps people to differentiate real news from fake news.

The content of fake news is rather diverse in terms of topics, styles, and media platforms. To help mitigate the adverse effects caused by fake news, it is of utmost importance to develop methods that automatically detect fake news on social media [14]. Although the language has no direct connection to distinguish real and fake news, legitimate and faked news articles use language differently in ways that can be detected by algorithms. The distinctive ways language is used to detect fake news have been explored by a number of research groups, such as [4], [8], [9], [14], [11] and [16]. In this article, we employ a deep learning based automated detector approach using a three-level Hierarchical Attention Network (HAN) accurate detection of fake news. Regarding the work of Monteiro et al. [9] that establishes a baseline classification metric using machine learning techniques, the present work tests the effectiveness of a HAN to detect deception on written texts. We also expect that by applying a HAN model, its attention layers could highlight the most relevant sentences for the classifier.

The remainder of this article is organized as follows. In Section 2, we start by overviewing related works on fake news detection. In Section 3, we briefly discuss the datasets and explain the network model adopted. The results are presented in Section 4, and we conclude in Section 5.

\section{Related Work}

As James Kershner pointed out in his book [7] "So what makes fake news fake? If news refers to an accurate account of a real event, what does fake news mean?" Actually, 'fake news' has become a buzzword. Nowadays, this term does not differentiate misinformation in the media from actually fabricated news, nor a news satire from large-scale hoaxes, rumors. Only recently, on an article by

\footnotetext{
${ }^{3}$ http://bit.ly/2og $4 \mathrm{zvV}$

${ }^{4}$ https://thetrustproject.org
} 
Tandoc Jr. and colleagues [16], the authors clearly identified the dimensions that guided previous definitions of fake news, offering a typology based on such dimensions that include news satire, news parody, news fabrication, advertising material, propaganda (referring to news stories created by a political entities), and even photo manipulation.

Earlier, Conroy, Rubin, and Chen [4] also propose a typology to asses veracity composed of methods emerging from two major categories, which are: linguistic cue approaches and network analysis approaches. They concluded proposing operational guidelines based on a hybrid approach that combine both previously cited categories. In another article [12], we understand that these same authors inaugurated a more computer methodological focused research on deception detection. They analyzed rhetorical structures, discourse constituent parts, and their coherence relations in deceptive and in real news samples. They further applied a vector space model to cluster the news by discourse feature similarity, achieving $63 \%$ accuracy.

Recently Pérez-Rosas and co-authors [11] focused on the automatic detection of fake online news. Their dualistic work consists of two novel datasets, covering seven different news topics, for fake news disclosure; and a set of learning experiments able to detect fake news achieving accuracies of up to $76 \%$. Some of the linguistic features are also presented in the work of Okano and Ruiz [13], where they tested 76 linguistic cues on the Fake.Br [9] parallel corpus and achieved accuracy results of $75 \%$ using support vector machines (SVM), random forest, and logistic regression. We address the work of Monteiro and colleagues [9] about the construction of the Fake.Br, the first Brazilian Portuguese parallel fake news corpus, later, on section 3.1 .

One of the first attempts to use HAN in social media was aimed to detect rumor in microblogs was reported by [5]. They build a hierarchical bidirectional long short-term memory (BLSTM) model for representation learning. The social contexts were incorporated into the network via attention mechanism in a way that that important semantic information was introduced to the framework for robust rumor detection. They achieved $94 \%$ using F1-measure on rumorous Weibo microblogs and $82.5 \%$ on tweets.

Hierarchical Attention Networks were first introduced in 2016 by Yang and collaborators [19], a partnership between Carnegie Mellon and Microsoft. As they emphasize, they propose this novel structure that mirrors the hierarchical structure of documents. This model incorporates the knowledge of document structure in the model architecture. It also has two levels of attention mechanisms that, when applied to the word and sentence-level, enable more or less importance to the content when constructing the document representation. They applied this new neural network on six large scale document classification data sets for two classification tasks: sentiment estimation and topic classification, and concluded that the proposed architecture outperforms previous methods by a significant margin.

Following Yang's article, we call attention to the work of Yang, Mukherjee, and Dragut [18] who used an attention mechanism and some linguistic features 
to recognize satirical cues at the paragraph level. They investigated the difference between paragraph-level features and document-level features and revealed what features are essential at which level. Singhania and collaborators [15] also have tested this three-level hierarchical attention network, which they called $3 \mathrm{HAN}$, but aiming for accurate detection of fake news. By their experiments, they argue that despite other deep learning models, the 3HAN model provides an 'understandable output through the attention weights given to different parts of an article'. In this paper, we innovate by proposing a HAN for document classification written in Brazilian Portuguese as disjoint labels of fake or real news, and also by adopting a categorical cross-entropy as loss function that measures the performance of the classification model in the $[0,1]$ interval.

\section{Dataset and methods}

In this section, we discuss the available dataset and present the algorithms used for fake news detection. We also focus on evaluation metrics for this task.

\subsection{Fake.Br Corpus}

The Fake.Br Corpus [9] is the first fake news reference corpus for Portuguese. It is composed of aligned pairs of authentic and fake news. This corpus is composed of 7,200 news, where 3,600 are fake news, and 3,600 are their real correspondent news. Table 1 shows the distributions of the news in each category.

\begin{tabular}{lcr}
\hline Category & $\begin{array}{c}\text { Samples } \\
\text { (\# of) }\end{array}$ & $\%$ \\
\hline Politics & 4180 & 58.0 \\
TV \& Celebrities & 1544 & 21.4 \\
Society \& Daily News & 1276 & 17.7 \\
Science \& Technology & 112 & 1.5 \\
Economy & 44 & 0.7 \\
Religion & 44 & 0.7
\end{tabular}

Table 1. Sampling distribution per category in Fake.BR. Adapted from Monteiro et. al. [9]

The authors manually collected and analyzed all the fake news from an interval of two years (from 01/2016 to 01/2018) from 4 websites, which are: Diário do Brasil, A Folha do Brasil, The Journal Brasil and Top Five TV, filtering out the news that presented half-truth. On the other hand, the authors collected the real news using a web crawler from three major Brazilian news agencies: G1, Folha de São Paulo, and Estadão. After collecting the news, the authors used a lexical similarity measure to choose the most similar real news to the fake news collected. They also performed a manual verification to guarantee that the fake news and real news were subject related. 
As the average number of tokens $\bar{t}$ in each corresponding class in Fake.Br is so big, fake news $\bar{t}=216.1$ tokens and true news $\bar{t}=1268.5$ tokens, the authors of the Fake.Br also offered a truncated version of the corpus in which both real and fake news, have the same number of characters based on the length (number of tokens) of the shortest of the pair. The HAN was applied to both corpora, the full, and the truncated one.

\subsection{Hierarchical Attention Network}

We adopt a general HAN architecture for document representation, displayed in Figure 1, initially proposed by Yang [19]. The overall architecture is represented in two major parts: a word-level attention layer, and a sentence-level attention layer, therefore representing two levels of abstraction. They are both preceded by the corresponding encoders, a word encoder, and a sentence encoder.

Word encoder Given a sentence with words $w_{i t}, t \in[1, T]$ for $T$ maximum number of words per sentence, they are embedded to vectors $x_{i T}$, see Equation 1. This model uses a bidirectional GRU network where the words are summarized in two directions, the forward hidden state $\overrightarrow{h_{i t}}$ and and backward hidden state $\overleftarrow{h_{i t}}$, (Equations 2 and 3 ) therefore summarizing the information of the whole sentence centered around $w_{i t}$, as in Equation 4.

Word attention layer The attention mechanism devised in the next phase (seen as $\alpha_{w}$ in Figure 1), extracts the most meaningful words to contribute to the sentence meaning. In other words, it gives weights to words considering the importance $\alpha_{i t}$ of the word $w_{i t}$ to the sentence $s_{i}$. In a summary, $u_{i t}$ resulted form a one layer MLP, as seen in Equation 5. Equation 6 represents the importance of the word, which is calculated using the softmax function to get the normalized importance weight $\alpha_{i t}$. Equation 7 refers to the output of the word attention layer, which is the sentence vector $s_{i}$, calculated as the weighted sum of the words annotation.

The following equations summarize both processes:

$$
\begin{aligned}
& x_{i t}=W_{e} w_{i t} \\
& \overrightarrow{h_{i t}}=\overrightarrow{\operatorname{GRU}}\left(x_{i t}\right) \text {, } \\
& \overleftarrow{h_{i t}}=\overleftarrow{\operatorname{GRU}}\left(x_{i t}\right) \text {, } \\
& h_{i t}=\left[\overrightarrow{h_{i t}}, \overleftarrow{h_{i t}}\right] \\
& u_{i t}=\tanh \left(W_{s} h_{i}+b_{s}\right) \\
& \alpha_{i t}=\frac{\exp \left(u_{i t}^{\top} u_{s}\right)}{\sum_{t} \exp \left(u_{i t}^{\top} u_{s}\right)} \\
& s_{i}=\sum_{t} \alpha_{i t} h_{i t}
\end{aligned}
$$

Recalling that $t \in[1, T]$ for all equations above.

Sentence encoder Similarly to the word encoder layer, we have the sentence encoder, which uses an analogous mechanism. Here we have sentence $s_{i}, i \in$ $[1, L]$, for $L$ the max number of sentences in a document. Equations 8-10 represent the sentence encoder mechanism and, similarly, with word encoder. Here $h_{i}$ stands for the information of the whole document centered around $s_{i}$. 
Sentence attention A similar attention mechanism was implemented to reward the sentences that are more important to the classification. Equations 11-13 can summarize the sentence attention layer.

$$
\begin{aligned}
& \overrightarrow{h_{i}}=\overrightarrow{\operatorname{GRU}}\left(s_{i}\right), i \in[1, L] \quad(8) \quad u_{i}=\tanh \left(W_{s} h_{i}+b_{s}\right) \\
& \overleftarrow{h_{i}}=\overleftarrow{\operatorname{GRU}}\left(s_{i}\right), i \in[L, 1] \\
& h_{i}=\left[\overrightarrow{h_{i}}, \overleftarrow{h_{i}}\right] \\
& \alpha_{i}=\frac{\exp \left(u_{i}^{\top} u_{s}\right)}{\sum_{i} \exp \left(u_{i}^{\top} u_{s}\right)} \\
& v=\sum_{i} \alpha_{i} h_{i}
\end{aligned}
$$

Document classification To classify the document we apply the document vector $v$ through a softmax function as shown in Equation 14, where $W_{c}$ is the weight vector and the $b_{c}$ is the bias vector.

$$
p=\operatorname{softmax}\left(W_{c} v+b_{c}\right)
$$

In our work, we used the categorical cross-entropy, Equation 15, as loss function. There the loss, $L$, is calculated as the sum of the entropy of all classes $M$, where $y_{c}$ is the class label, and $p_{c}$ is the predicted label.

$$
L=-\sum_{c=1}^{M} y_{c} \log p_{c}
$$

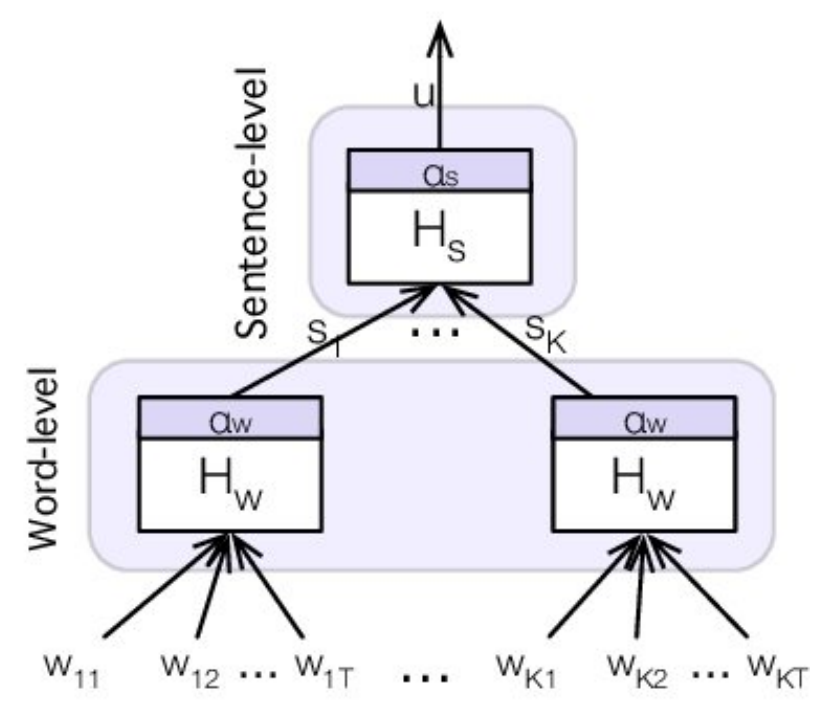

Fig. 1. General architecture of hierarchical attention neural networks for modeling documents. Reprinted from Pappas Popescu-Belis [10] 


\subsection{Model configuration}

Initially, we performed the preprocessing of the text, converting it to lowercase and removing or not the stop words(NLTK stop words). We then split the documents into sentences using a sentence tokenizer function (NLTK [2]). Each sentence was also split into tokens using the Keras [3] tokenizer.

In the word embedding layer, we used Hartmann et. al. [6] pre-trained Global Vectors (GloVe). Actually trained in a large Portuguese multi-genre corpus. In our experiments, we trained models utilizing GloVe with different dimensions $(d=\{100,300,600\})$.

For training, we used a batch size of 400, using the Keras Adam optimizer using the default parameters. The models were trained using this configuration during 100 epochs or until the loss did not decrease by at least $10^{-3}$ for ten successive epochs.

To evaluate the performance of the Hierarchical Attention Network in the task of detection of fake news, we used the same methodology used by Monteiro et.al. [9], running the algorithm in two setups: using full texts and using truncated texts. We used the 5 -fold cross-validation to evaluate the algorithm calculating the precision, recall, and F-measure metrics for each class, as well as overall accuracy.

\section{Results}

We performed our experiments using the full texts and the truncated texts. We chose the truncated datasets due to the difference in the number of tokens between the two classes. The average number of tokens in real news is 1,268.5, and in fake news is 216.1 .

In Table 2, we show the results obtained using the full-text dataset, where in the first column we present the setup used where GloVe $x$ are the results obtained using GloVe of $x$ dimensions and SW we removed all stop words. Looking at the results, we can observe that we obtained almost the same result using all the different configurations we proposed: Removing or not the stop words and using different numbers of dimensions of the GloVe model.

Using this model in the full texts, we obtained $97 \%$ of accuracy. Considering the significant difference in the number of tokens between the two classes to make this classification task more manageable, we checked the performance of this model in the truncated texts too.

In Table 3, we present the results using the truncated texts. There we can observe that the results obtained using HAN were close, or slightly better, than the results obtained by Monteiro et al. [9], when they mainly used bag of words to obtain an accuracy of $89 \%$ in both, fake and real class. One advantage of this model, to be pursued during future studies, is that we can extract an attention map from the attention layers showing the importance of each sentence and each word. 


\begin{tabular}{lccccccc}
\hline & \multicolumn{2}{c}{ Precision } & \multicolumn{2}{c}{ Recall } & \multicolumn{2}{c}{ F-score } & \multirow{2}{*}{ Accuracy } \\
& Fake & True & Fake & True & Fake & True & \\
\hline GloVe 100 & 0.9655 & 0.9695 & $\mathbf{0 . 9 6 9 4}$ & 0.9653 & 0.9674 & 0.9673 & 0.9674 \\
GloVe 100 SW & 0.9696 & 0.9532 & 0.9522 & 0.9700 & 0.9608 & 0.9615 & 0.9611 \\
GloVe 300 & 0.9666 & 0.9622 & 0.9619 & 0.9667 & 0.9642 & 0.9644 & 0.9643 \\
GloVe 300 SW & 0.9672 & 0.9640 & 0.9639 & 0.9672 & 0.9655 & 0.9656 & 0.9656 \\
GloVe 600 & $\mathbf{0 . 9 7 0 6}$ & $\mathbf{0 . 9 6 9 6}$ & $\mathbf{0 . 9 6 9 4}$ & $\mathbf{0 . 9 7 0 6}$ & $\mathbf{0 . 9 7 0 0}$ & $\mathbf{0 . 9 7 0 0}$ & $\mathbf{0 . 9 7 0 0}$ \\
GloVe 600 SW & 0.9655 & 0.9632 & 0.9631 & 0.9656 & 0.9643 & 0.9643 & 0.9643 \\
\hline
\end{tabular}

Table 2. Results of the HAN applied on full texts.

\begin{tabular}{lccccccc}
\hline & \multicolumn{2}{c}{ Precision } & \multicolumn{2}{c}{ Recall } & \multicolumn{2}{c}{ F-score } & \multirow{2}{*}{ Accuracy } \\
& Fake & True & Fake & True & Fake & True & \\
\hline GloVe 100 & 0.8966 & $\mathbf{0 . 9 0 3 8}$ & $\mathbf{0 . 9 0 4 4}$ & 0.8956 & 0.9004 & 0.8996 & 0.9000 \\
GloVe 100 SW & 0.8801 & 0.8884 & 0.8892 & 0.8769 & 0.8840 & 0.8819 & 0.8831 \\
GloVe 300 & 0.9035 & 0.9018 & 0.9017 & 0.9036 & 0.9026 & 0.9027 & 0.9026 \\
GloVe 300 SW & 0.8808 & 0.8983 & 0.9003 & 0.8764 & 0.8899 & 0.8865 & 0.8883 \\
GloVe 600 & $\mathbf{0 . 9 0 9 4}$ & 0.9026 & 0.9014 & $\mathbf{0 . 9 1 0 3}$ & $\mathbf{0 . 9 0 5 3}$ & $\mathbf{0 . 9 0 6 4}$ & $\mathbf{0 . 9 0 5 8}$ \\
GloVe 600 SW & 0.8999 & 0.8990 & 0.8989 & 0.9000 & 0.8994 & 0.8995 & 0.8994 \\
\hline
\end{tabular}

Table 3. Results of the HAN applied on truncated texts.

\section{Conclusion}

We believe the enormous spread of fake news came along with the increasing popularity of social media. In this article, we explored the problem of fake news detection by reviewing the hierarchical attention networks applied to texts written in Brazilian Portuguese. Previously the HAN model has been successfully applied to sentiment analysis [19], where the attention layers were able to select qualitatively informative words and sentences. We expected a similar behavior for this model when applied to distinguish between fake and real news, which did not happen for this corpus. Besides the narrow margin of contrast between fake and real news presented by this deep learning model, this architecture poses a high computing cost to obtain similar results as other comparable lower-cost architectures such as machine learning models [9].

For future work, we want to use the metadata provided in Fake.Br corpus to improve the classification results and also explore the attention map extracted from these texts to verify the importance of each word and sentence to the classification of fake and true news, we also want to use this model in another classification task as well as sentiment analysis in Portuguese texts.

\section{Acknowledgements}

This research was supported by Fundação de Amparo à Pesquisa do Estado de São Paulo (FAPESP), process number 2018/03129-8. 


\section{References}

1. Allcott, H., Gentzkow, M.: Social media and fake news in the 2016 election. Journal of Economic Perspectives 31(2), 211-36 (2017)

2. Bird, S., Klein, E., Loper, E.: Natural Language Processing with Python: Analyzing text with the natural language toolkit. O'Reilly Media, Inc. (2009)

3. Chollet, F., et al.: Keras. https://keras.io (2015)

4. Conroy, N.J., Rubin, V.L., Chen, Y.: Automatic deception detection: Methods for finding fake news. Proceedings of the Association for Information Science and Technology 52(1), 1-4 (2015)

5. Guo, H., Cao, J., Zhang, Y., Guo, J., Li, J.: Rumor Detection with Hierarchical Social Attention Network. In: Proceedings of the 27th ACM International Conference on Information and Knowledge Management. pp. 943-951. CIKM'18, ACM, New York, NY, USA (2018). https://doi.org/10.1145/3269206.3271709, http://doi.acm.org/10.1145/3269206.3271709

6. Hartmann, N., Fonseca, E., Shulby, C., Treviso, M., Rodrigues, J., Aluisio, S.: Portuguese word embeddings: Evaluating on word analogies and natural language tasks. arXiv preprint arXiv:1708.06025 (2017)

7. Kershner, J.W.: Elements of News Writing. Allyn and Bacon, Boston, MA (2004)

8. Lazer, D.M., Baum, M.A., Benkler, Y., Berinsky, A.J., Greenhill, K.M., Menczer, F., Metzger, M.J., Nyhan, B., Pennycook, G., Rothschild, D., et al.: The science of fake news. Science 359(6380), 1094-1096 (2018)

9. Monteiro, R.A., Santos, R.L.S., Pardo, T.A.S., de Almeida, T.A., Ruiz, E.E.S., Vale, O.A.: Contributions to the study of fake news in portuguese: New corpus and automatic detection results. In: Villavicencio, A., Moreira, V., Abad, A., Caseli, H., Gamallo, P., Ramisch, C., Gonçalo Oliveira, H., Paetzold, G.H. (eds.) Computational Processing of the Portuguese Language. pp. 324-334. Springer International Publishing, Cham (2018)

10. Pappas, N., Popescu-Belis, A.: Multilingual hierarchical attention networks for document classification. arXiv preprint arXiv:1707.00896 (2017)

11. Pérez-Rosas, V., Kleinberg, B., Lefevre, A., Mihalcea, R.: Automatic Detection of Fake News. In: Proceedings of the 27th International Conference on Computational Linguistics. pp. 3391-3401. Association for Computational Linguistics, Santa Fe, New Mexico, USA (Aug 2018), https://www.aclweb.org/anthology/C18-1287

12. Rubin, V.L., Conroy, N.J., Chen, Y.: Towards news verification: Deception detection methods for news discourse. In: Hawaii International Conference on System Sciences (HICSS48) Symposium on Rapid Screening Technologies, Deception Detection and Credibility Assessment Symposium. Grand Hyatt, Kauai, Hawaii (2015). https://doi.org/10.13140/2.1.4822.8166

13. Ruiz, E.E.S., Okano, E.Y.: Using linguistic cues to detect fake news on the Brazilian Portuguese parallel corpus Fake.BR . In: Proceedings of the 12th Brazilian Symposium in Information and Human Language Technology (2019)

14. Shu, K., Sliva, A., Wang, S., Tang, J., Liu, H.: Fake news detection on social media: A data mining perspective. ACM SIGKDD Explorations Newsletter 19(1), 22-36 (2017)

15. Singhania, S., Fernandez, N., Rao, S.: 3HAN: A deep neural network for fake news detection. In: International Conference on Neural Information Processing. pp. 572 581. Springer (2017)

16. Tandoc Jr., E.C., Lim, Z.W., Ling, R.: Defining "fake news": A typology of scholarly definitions. Digital Journalism 6(2), 137-153 (2018) 
17. Wakefield, A.J.: MMR vaccination and autism. The Lancet 354(9182), 949-950 (1999)

18. Yang, F., Mukherjee, A., Dragut, E.: Satirical news detection and analysis using attention mechanism and linguistic features. arXiv preprint arXiv:1709.01189 (2017)

19. Yang, Z., Yang, D., Dyer, C., He, X., Smola, A., Hovy, E.: Hierarchical attention networks for document classification. In: Proceedings of the 2016 Conference of the North American Chapter of the Association for Computational Linguistics: Human Language Technologies. pp. 1480-1489 (2016) 